\title{
EXACT AND HEURISTIC ALGORITHMS FOR SOLVING THE GENERALIZED MINIMUM FILTER PLACEMENT PROBLEM
}

by

Enock Chisonge Mofya

\author{
A Dissertation Submitted to the Faculty of the \\ DEPARTMENT OF SYSTEMS AND INDUSTRIAL ENGINEERING \\ In Partial Fulfillment of the Requirements \\ For the Degree of \\ DOCTOR OF PHILOSOPHY \\ In the Graduate College \\ THE UNIVERSITY OF ARIZONA
}

2005 


\section{THE UNIVERSITY OF ARIZONA ® GRADUATE COLLEGE}

As members of the Dissertation Committee, we certify that we have read the dissertation prepared by Enock Chisonge Mofya entitled Exact and Heuristic Algorithms for Solving the Generalized Minimum Filter Placement Problem and recommend that it be accepted as fulfilling the dissertation requirement for the Degree of Doctor of Philosophy

\begin{tabular}{|c|c|}
\hline \multirow{3}{*}{ J. Cole Smith } & $10 / 07 / 05$ \\
\hline & Date \\
\hline & $10 / 07 / 05$ \\
\hline \multirow[t]{2}{*}{ Ronald G. Askin } & Date \\
\hline & $10 / 07 / 05$ \\
\hline \multirow[t]{2}{*}{ Mirchandani B. Pitu } & Date \\
\hline & $10 / 07 / 05$ \\
\hline \multirow[t]{2}{*}{ Bruce J. Bayly } & Date \\
\hline & $10 / 07 / 05$ \\
\hline
\end{tabular}

Final approval and acceptance of this dissertation is contingent upon the candidate's submission of the final copies of the dissertation to the Graduate College.

I hereby certify that I have read this dissertation prepared under my direction and recommend that it be accepted as fulfilling the dissertation requirement. 


\section{STATEMENT BY AUTHOR}

This dissertation has been submitted in partial fulfillment of requirements for an advanced degree at The University of Arizona and is deposited in the University Library to be made available to borrowers under rules of the Library.

Brief quotations from this dissertation are allowable without special permission, provided that accurate acknowledgment of source is made. Requests for permission for extended quotation from or reproduction of this manuscript in whole or in part may be granted by the head of the major department or the Dean of the Graduate College when in his or her judgment the proposed use of the material is in the interests of scholarship. In all other instances, however, permission must be obtained from the author.

SIGNED: E. Chisonge Mofya 


\section{ACKNOWLEDGEMENTS}

I would like to express my sincere and heartfelt gratitude to my advisor, Dr. Jonathan Cole Smith, for his support, patience, encouragement and financial support he provided for me throughout my $\mathrm{Ph}$.D. studies. It is because of his vision and great insight that this study has been possible. I would also like to extend my thanks to Dr. Ronald G. Askin, Dr. Bruce J. Bayly, Dr. Robert A. Indik, and Dr. Pitu B. Mirchandani, for their willingness to serve on my committee. I especially thank my major committee members, Dr. Askin and Dr. Mirchandani for reading the previous drafts of this dissertation and providing valuable and instructive comments.

I would also like to express my appreciation for the help and encouragement my colleagues and friends provided for me during my study. I'm particularly grateful to Lewis Ntaimo for having encouraged me to take classes from the SIE department. He is partly the reason I pursued my Ph.D. study in Systems and Industrial Engineering. He encouraged me to take classes from Dr. Smith; this I did and before I knew it, I was talking to Dr. Smith about the possibility of my doing graduate studies under him. The rest is history. I extend my gratitude to Jiaqiong Chen, Dale Henderson, Lei Zhao, and Josephat Zimba for the numerous research related discussions I had with each one of them on separate occasions.

This research was funded by grants from the Office of Naval Research (N0001403-1-0510) and the Defence Advanced Research Projects Agency (N66001-01-18925). 


\section{DEDICATION}

To my wife Charity Tsitsi Mofya and our lovely son Mwandama Elijah Mofya who was born during my Ph.D. studies. And to my father Elijah Chisonge Mofya, and to all my family members. Your love, understanding and encouragement helped me through my long years of study. Thank you for seeing me through. 


\section{TABLE OF CONTENTS}

LIST OF FIGURES .................... 8

LIST OF TABLES ......................... 9

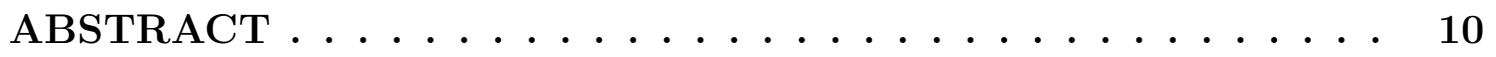

CHAPTER 1 Introduction ................ 11



1.2 Problem Statement . . . . . . . . . . . . . . . . . . . 12

1.3 Research Scope and Approach . . . . . . . . . . . . . . . . . 13

1.4 Organization of the dissertation . . . . . . . . . . . . . 14

CHAPTER 2 Literature Review .............. 15

2.1 Flooding-based DDoS Attacks . . . . . . . . . . . . . . 15

2.2 Defense Mechanisms Against DDoS Attacks . . . . . . . . . . . . . . 18

2.3 Defending Against Flooding-based DDoS Attacks with Route-based Packet Filters . . . . . . . . . . . . . . . . . . . . . . 20

2.4 The Distributed Packet Filter Placement Problem . . . . . . . . . . . 24

CHAPTER 3 Mixed-Integer Programming Model and a Greedy Vertex Cover Heuristic for the GMFPP . . . . . . . . . . . 27

3.1 The GMFPP: Definition and Notation. . . . . . . . . . . . . . . 27

3.2 Mixed-Integer Programming Model . . . . . . . . . . . . . . . . . . . 28

3.3 The Extended Vertex Cover Greedy Heuristic . . . . . . . . . . . . . 31

3.4 Computational Results . . . . . . . . . . . . . . . . . . 32

3.5 Summary . . . . . . . . . . . . . . . . . . . 39

CHAPTER 4 The GMFPP on Special Network Topologies. . . . . 40

4.1 Structures That Admit Closed-Form Solutions . . . . . . . . . . . . 41

4.2 Tree Networks . . . . . . . . . . . . . . . . . . . 43

4.2.1 Dynamic Programming Notation . . . . . . . . . . . . . 43

4.2.2 Dynamic programming algorithm . . . . . . . . . . . . . 44

4.2.3 Complexity of the dynamic program . . . . . . . . . . . 54

4.3 Computational Results . . . . . . . . . . . . . . 54

4.4 Summary . . . . . . . . . . . . . . . . . . 58 


\section{TABLE OF CONTENTS - Continued}

CHAPTER 5 Valid Inequalities and Heuristic Algorithms for the

GMFPP. ................... 59

5.1 Independent Subgraphs and Valid Inequalities . . . . . . . . . . 59

5.2 A Dynamic Programming Algorithm for Solving In-tree Subgraphs . . 64

5.3 Heuristic Algorithms . . . . . . . . . . . . . . . . . . 70

$5.3 .1 \quad$ LP-based Heuristic . . . . . . . . . . . . . . . . . . 70

5.3.2 Dynamic Programming-based Heuristic . . . . . . . . . . 71

5.3.3 Greedy Multiple Restart Heuristic . . . . . . . . . . . . . . . 73

5.4 Computational Tests . . . . . . . . . . . . . . . . . . 75

5.4 .1 Generation of Instances . . . . . . . . . . . . . . . . 75

5.4 .2 Computational Results . . . . . . . . . . . . . . 77

5.5 Summary . . . . . . . . . . . . . . . . . . 96

CHAPTER 6 Conclusions and Future Work $\ldots \ldots \ldots$. . . . 97

6.1 Conclusions and Summary . . . . . . . . . . . . . . . . . 97

6.2 Future Work . . . . . . . . . . . . . . . . . . . . . . 98

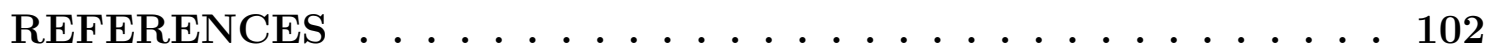




\section{LIST OF FIGURES}

2.1 DDoS attack network hierarchy. . . . . . . . . . . . . . . 17

2.2 DDoS attack network hierarchy. . . . . . . . . . . . . . . 18

2.3 A route-based filter placed on node 8 will stop an attack on node 2 from node 10 with forged node 4 address. . . . . . . . . . . . . . . . . 21

2.4 Maximal versus semi-maximal filtering example. . . . . . . . . . . . 23

3.1 Mixed-integer Programming Solution, with $b=3 . \ldots 33$

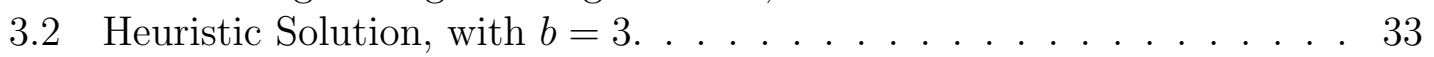

3.3 Small instance set. (a) average Cpu times vs traceback number. (b) standard deviation of computational times vs traceback number. . . . 34

3.4 Medium instance set. (a) average $\mathrm{Cpu}$ times vs traceback number. (b) standard deviation of computational times vs traceback number. . 35

3.5 Large instance set. (a) average Cpu times vs traceback number. (b) standard deviation of computational times vs traceback number. . . . 35

4.1 An illustration of the tree network topology. . . . . . . . . . . . . . . 44

4.2 The process of node aggregation. . . . . . . . . . . . . . 45

5.1 The process of node aggregation for in-tree subgraphs. . . . . . . . 66

5.2 Schematic of the LP-based heuristic. . . . . . . . . . . . . . . . 70

5.3 Schematic of the DP1 heuristic. . . . . . . . . . . . . . . . . 72

5.4 Schematic of the DP2 heuristic. . . . . . . . . . . . . . . 73

5.5 CPU seconds versus total suboptimality for the instance set Inst_20. . 94

5.6 CPU seconds versus total suboptimality for the instance set Inst_30. . 94

5.7 CPU seconds versus total suboptimality for the instance set Inst_40. . 95

6.1 Basic constructs of workflow models. . . . . . . . . . . . . . . . 99

6.2 Split and join routing structures. . . . . . . . . . . . . . . . . . 99

6.3 An example of a deadlock. . . . . . . . . . . . . . . . . . . . 100

6.4 An example of a lack of synchronization. . . . . . . . . . . . . . . . 101 


\section{LIST OF TABLES}

3.1 Quality of heuristic solutions compared to optimal solutions for the small instance set. . . . . . . . . . . . . . . . 36

3.2 Quality of heuristic solutions compared to optimal solutions for the medium instance set. . . . . . . . . . . . . . . . . 37

3.3 Quality of heuristic solutions compared to optimal solutions for the large instance set. . . . . . . . . . . . . . . . 38

4.1 Example of a matrix $M \ldots \ldots \ldots \ldots \ldots \ldots$

4.2 Average CPU times and standard deviations for Inst_15, in seconds. . 56

4.3 Average CPU times and standard deviations for Inst_30, in seconds. . 57

4.4 Average CPU times and standard deviations for Inst_45, in seconds. . 57

4.5 Average CPU seconds required to solve instance sets with 100, 150, 200, and 250 nodes. . . . . . . . . . . . . 58

5.1 Average CPU times for Inst 15 in seconds. . . . . . . . . . . . . . 81

5.2 Average CPU times for Inst 20 in seconds. . . . . . . . . . . . . 81

5.3 Average CPU times for Inst 25 in seconds. . . . . . . . . . . . . 81

5.4 Average CPU times for Inst_30 in seconds. . . . . . . . . . . . . . 82

5.5 Average CPU times for Inst_35 in seconds. . . . . . . . . . . . . . . 82

5.6 Average CPU times for Inst_40 in seconds. . . . . . . . . . . . . 83

5.7 Average CPU times for Inst_30_ind_20 in seconds. . . . . . . . . . . . 83

5.8 Average CPU seconds for Inst_15 with DP inequalities. . . . . . . . . 84

5.9 Average CPU seconds for Inst_20 with DP inequalities. . . . . . . . . 84

5.10 Average CPU seconds for Inst_25 with DP inequalities. . . . . . . . . 85

5.11 Average CPU seconds for Inst_30 with DP inequalities. . . . . . . . . 85

5.12 Average CPU seconds for Inst_35 with DP inequalities. . . . . . . . 86

5.13 Average CPU seconds for Inst_40 with DP inequalities. . . . . . . . . 87

5.14 Heuristic suboptimality for Inst_15 . . . . . . . . . . . . . 88

5.15 Heuristic suboptimality for Inst_20 . . . . . . . . . . . . . . . 89

5.16 Heuristic suboptimality for Inst $25 \ldots \ldots$. . . . . . . . . 90

5.17 Heuristic suboptimality for Inst_30 . . . . . . . . . . . . . . 91

5.18 Heuristic suboptimality for Inst_35 . . . . . . . . . . . . . . 92

5.19 Heuristic suboptimality for Inst_40 . . . . . . . . . . . . . 93

5.20 Heuristic average CPU times in seconds. . . . . . . . . . . . . . 93 


\begin{abstract}
We consider a problem of placing route-based filters in a communication network to limit the number of forged address attacks to a prescribed level. Nodes in the network communicate by exchanging packets along arcs, and the originating node embeds the origin and destination addresses within each packet that it sends. In the absence of a validation mechanism, one node can send packets to another node using a forged origin address to launch an attack against that node. Route-based filters can be established at various nodes on the communication network to protect against these attacks. A route-based filter examines each packet arriving at a node, and determines whether or not the origin address could be legitimate, based on the arc on which the packet arrives, the routing information, and possibly the destination. The problem we consider seeks to find a minimum cardinality subset of nodes to filter so that the prescribed level of security is achieved.

The primary contributions of this dissertation are as follows. We formulate and discuss the modeling of this filter placement problem as a mixed-integer program. We then show the sensitivity of the optimal number of deployed filters as the required level of security changes, and demonstrate that current vertex cover-based heuristics are ineffective for problems with relaxed security levels. We identify a set of special network topologies on which the filter placement problem is solvable in polynomial time, focusing our attention on the development of a dynamic programming algorithm for solving this problem on tree networks. These results can then in turn be used to derive valid inequalities for a mixed-integer programming model of the filter placement problem. Finally, we present heuristic algorithms based on the insights gained from our overall study for solving the problem, and evaluate their performance against the optimal solution provided by our mixed-integer programming model.
\end{abstract}




\section{CHAPTER 1}

\section{Introduction}

\subsection{Motivation}

In recent years, a significant amount of research has been devoted to developing security measures aimed at alleviating vulnerabilities inherent in transportation and communication networks. One common approach towards achieving this goal is to concentrate on verifying the authenticity of an entity given its origin, current position in the network, and final destination. This approach typically employs a system of authenticating units strategically distributed throughout the network. Generally, the more authenticating units there are in the network, the more secure the network is. However, there is a tradeoff between the number of authenticating units and the smooth running of the network. Increasing the number of authenticating units tends to slow, or even disrupt, the normal operations of the network. Therefore, it is imperative that we use the absolute minimum number of authenticating units needed to achieve the required level of security.

In the realm of internet communication, one of the most pressing security problems that falls into this category is the Denial of Service (DoS) attack. While there are many variants of DoS attacks, they all serve the sole purpose of disrupting the services offered by the victim $[28,35,38,45]$. When attacks are orchestrated from different locations, they are called Distributed Denial of Service attacks $[11,29]$. DDoS attacks have caused e-commerce significant losses in revenues by disrupting critical network services. For instance, in early February of 2000, DDoS attacks were launched against major Web sites including Amazon.com, Buy.com, CNN.com, Ebay.com, E-Trade.com, and ZDNet.com. According to the Yankee Group estimates, the cumulative cost of the attack totaled $\$ 1.2$ billion while the cost to Amazon.com alone was estimated between $\$ 200,000$ and $\$ 300,000$ per hour 
$[75]$.

\subsection{Problem Statement}

In this dissertation, we consider a class of communication network attacks described by the following scenario. Let $G=(N, A)$ be a directed graph representing a communication network in which $N=\{1, \ldots, n=|N|\}$ is a set of nodes and $A$ is a set of arcs. Nodes represent communication centers and arcs represent message pathways. Nodes communicate by exchanging packets along arcs, where each packet is specified with an origin address and a destination address. However, a node $s \in N$ may send a series of communication requests to another node $d \in N$ with a forged address of $o \in N$. Node $d$ has no choice but to ignore requests from $o$, which effectively disables communication from $o$ to $d$. If $s$ chooses to forge its address using multiple false origin addresses, it can practically shut down the effective operation of node $d$ altogether.

In Internet communication such attacks are called flooding-based DoS attacks (and flooding-based DDoS attacks, if they are conducted from multiple locations). Filtering mechanisms that mitigate DoS attacks include ingress/egress filtering [29, 69], probabilistic packet filtering [62, 70], and route-based packet filtering [60, 63]. The study presented in this dissertation focus on the deployment of route-based filters (and hence all references to packet filters represent route-based filters).

Route-based filters are placed at a subset of the nodes, and function by analyzing traffic in a network to verify whether the purported origin address on communication packets entering filtered nodes can be legitimate. If a filter can determine that a packet has a forged address, it drops the packet from the network, and otherwise it lets the packet proceed on its route. A packet having a valid origin address will never be dropped by a filter; this requirement is called safety. In the context of Internet communication, the process of locating an attack source is called IP Traceback [3, 6, 49, 73].

The problem of finding a minimum cardinality subset of nodes on a communi- 
cation network so that placing route-based filters on these nodes ensures that any packet with a forged origin address will be dropped by the deployed filters was formally studied in [4]. The authors proved that the problem is strongly NP-hard and demonstrated that many filters must typically be deployed under such stringent security requirements.

The work presented in this dissertation generalizes the study in [4]. Instead of requiring perfect security, we may wish to simply bound the amount of work necessary for a node under attack to determine the attacking node. For instance, suppose that for any node $d$ currently under attack from purported node $o$, the true origin of the attack must come from no more than $b$ nodes. In this case, node $d$ can investigate these $b$ candidates one-by-one to ascertain which node is attacking. We refer to this problem as the Generalized Minimum Filter Placement Problem (GMFPP).

\subsection{Research Scope and Approach}

We study the GMFPP in this dissertation. We start by evaluating the performance of the greedy vertex cover heuristic presented in [4] against mixed-integer programming solutions at varied security levels. We then focus our attention on identifying polynomially solvable special cases of the GMFPP. We develop a dynamic programming algorithm that solves the GMFPP on tree networks in polynomial-time. We employ results we derived for polynomially solvable special network topologies to derive valid inequalities for the GMFPP on a general network topology. We also develop efficient heuristic algorithms for solving the GMFPP.

All algorithmic implementations discussed in this dissertation were done using the $\mathrm{C}++$ programming language. Mixed-integer programming models were implemented using CPLEX 8.1 via ILOG Concert Technology 1.3, and all computations were run on a SUN UltraSparc-III with a $900 \mathrm{MHz}$ processor and 2.0 GB RAM. 


\subsection{Organization of the dissertation}

The rest of the dissertation is organized as follows. In Chapter 2 we provide a literature review of related work. We review the nature of DDoS attacks and some common defense mechanisms against these attacks. We provide a mixed-integer programming model of the GMFPP in Chapter 3, and use the optimal solution as a benchmark to evaluate the performance of a greedy vertex cover heuristic when applied to the GMFPP. In Chapter 4, we identify special network topologies that can be solved in polynomial time. We also develop a polynomial-time dynamic programming algorithm for solving the GMFPP on tree networks. We derive valid inequalities for the GMFPP on a general network in Chapter 5 using results from Chapter 4. In addition, we develop heuristic algorithms for estimating optimal solutions to the GMFPP. Finally we conclude with a summary of our work and suggestions for future work in Chapter 6. 


\section{CHAPTER 2}

\section{Literature Review}

In this chapter we review some literature related to the work presented in this dissertation. We start with a discussion of flooding-based DDoS attacks in Section 2.1. We give a brief review of defense mechanisms against DDoS attacks that have been proposed in the literature in Section 2.2, and finally we give a detailed recapitulation of the use of route-based filters to defend against flooding-based DDoS attacks in Section 2.3.

\subsection{Flooding-based DDoS Attacks}

DDoS attacks can be classified into different categories depending on the weakness in the communication network exploited, the part of communication protocol compromised, or the manner in which attacks are perpetrated [26, 40, 42, 71]. For instance, Douligeris and Mitrokotsa [26] classify DDoS attacks based on exploited vulnerability into four categories briefly summarized here: flood attacks, amplification attacks, protocol exploit attacks, and malformed packet attacks.

In a flood attack, a large volume of packets are sent to a victim system in order to disrupt the victim's ability to serve legitimate packets. The effect of the attack packet stream varies from slowing down the victim system to crashing it due to the resulting saturation of the network bandwidth.

In amplification attacks, the attacker exploits the broadcast IP address feature found in most routers. The attacker sends packets requiring a response to the router to be broadcasted. However, all the packets have a spoofed IP address of the victim system. When the router broadcasts the packets, all systems within the router's broadcasting range direct their responses to the victim system, thereby amplifying the effect of the attack by the number of responding systems. This could potentially 
saturate the victim's bandwidth and hamper its ability to serve legitimate requests.

Protocol exploit attacks exploit features or implementation bugs of some protocol installed on the victim in order to consume excess amounts of resources. A typical example of protocol exploit attacks is TPC SYN attacks. These attacks exploit the three-way handshake involved in a TCP connection process. A client initiates the connection process by sending a SYN (synchronize/start) request to a server. The server responds by sending back a SYN/ACK (synchronize/acknowledge) packet and waits for the final ACK (acknowledge) to complete the connection. The server maintains the half-open connections until either the final ACK is received, or the TCP connection times out after a preset amount of time. In a TCP SYN flooding attack, the attacker aims at exhausting the victim's TCP connection resources by sending a large number of SYN packets with spoofed IP addresses. Since the SYN packets are spoofed, the server never receives the final ACK messages. Due to the limited buffer queue for new connections, the server eventually is unable to process other incoming connections as the queue gets overloaded [19].

Finally, malformed packet attacks rely on incorrectly formed IP packets that are sent to the victim in order to crush the victim's system. There are at least two types of malformed packet attacks [74]: IP address attack and IP packet option attack. In the IP address attack, the packet contains the same source and destination IP addresses. This has the effect of confusing the operating system of the victim system and may cause it to crash. In the IP packet options attack, the malformed packet may randomize the optional fields within the IP packet and set all quality of service bits to one. As a result, the victim system must use additional processing time to analyze the traffic. If large amounts of such packets are sent to the victim system, the victim's processing ability may be depleted, resulting in the system crash.

In this chapter, we focus our attention on flooding-based DDoS attacks which are closely related to the study presented in this dissertation. Flooding-based DDoS attacks can broadly be classified into two categories: direct attacks and reflector attacks [13]. In direct attacks, the attacker typically sets up an attack network consisting of compromised systems [13, 59]. The attacker configures the attack 
network into a hierarchical structure as shown in Figure 2.1. The attacker occupies the highest level of the hierarchy and controls machines at the next level. These machines are often referred to as masters or clients. Each master controls a subset of machines at the lowest level often referred to as agents or zombies. Zombies are machines that carries out the actual DDoS attacks against the victim system. They lay dormant until they receive an attack command from the master machine detailing them with the victim's address, attack method, and attack duration. The victim's system can be severely hampered or even crushed by the attack, depending on the number of zombies. To avoid easy identification, zombies usually conceal their true identity by using spoofed IP addresses.

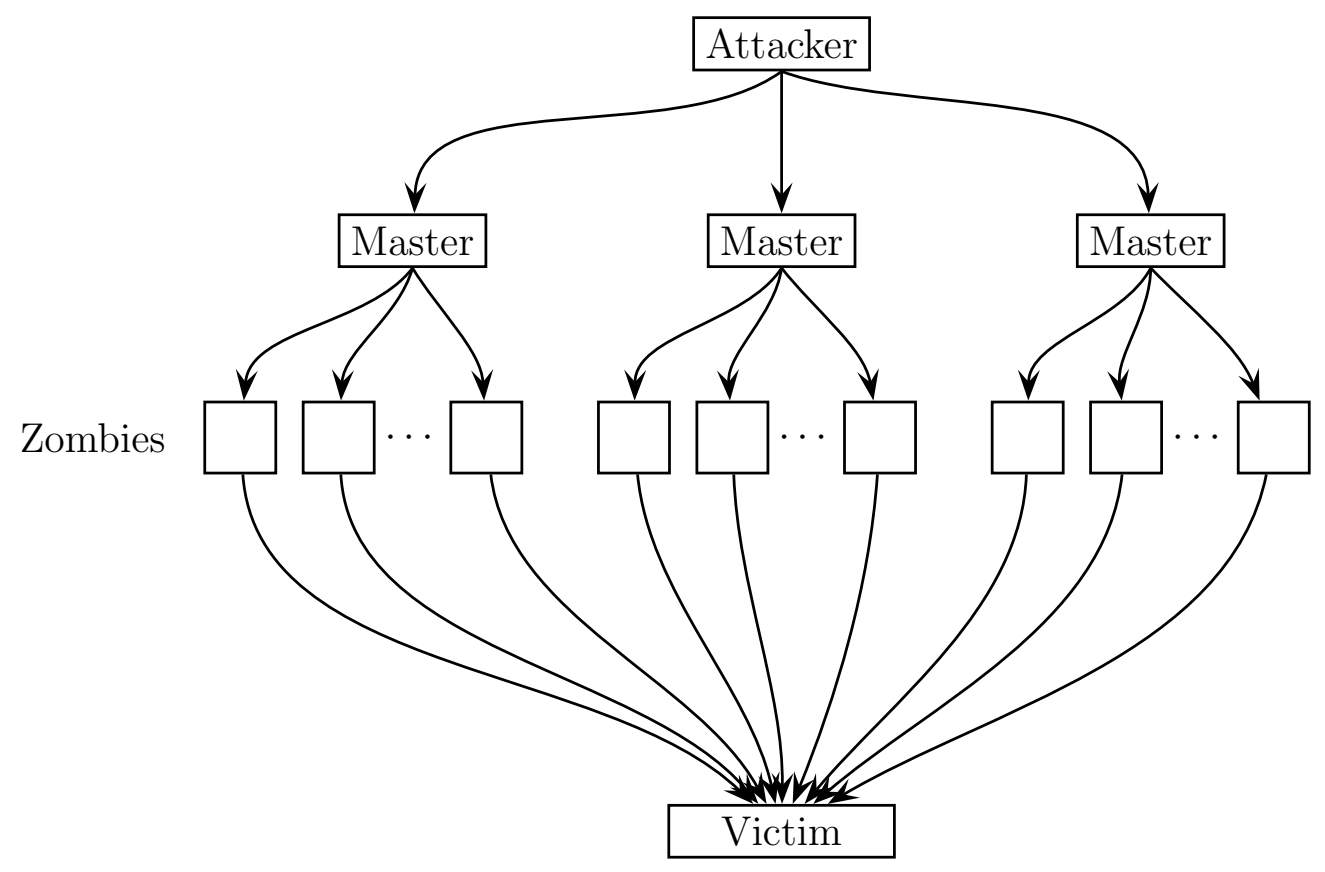

Figure 2.1: DDoS attack network hierarchy.

In reflector attacks, the attacker employs intermediate unsuspecting machines called reflectors to launch the actual attack on the victim. The attacker sends packets that require a response with a spoofed victim's address as the origin to a number of reflectors. When reflectors respond to these packets, they direct their 
response to the victim machine. If the number of reflectors is large enough, the victim's network can be overwhelmed with the amount of traffic. The effects of the attack are even more devastating if an attack network network (as opposed to a single attacking node) is used to send packets to reflectors [64]. Figure 2.2 gives a schematic representation of reflector attacks.

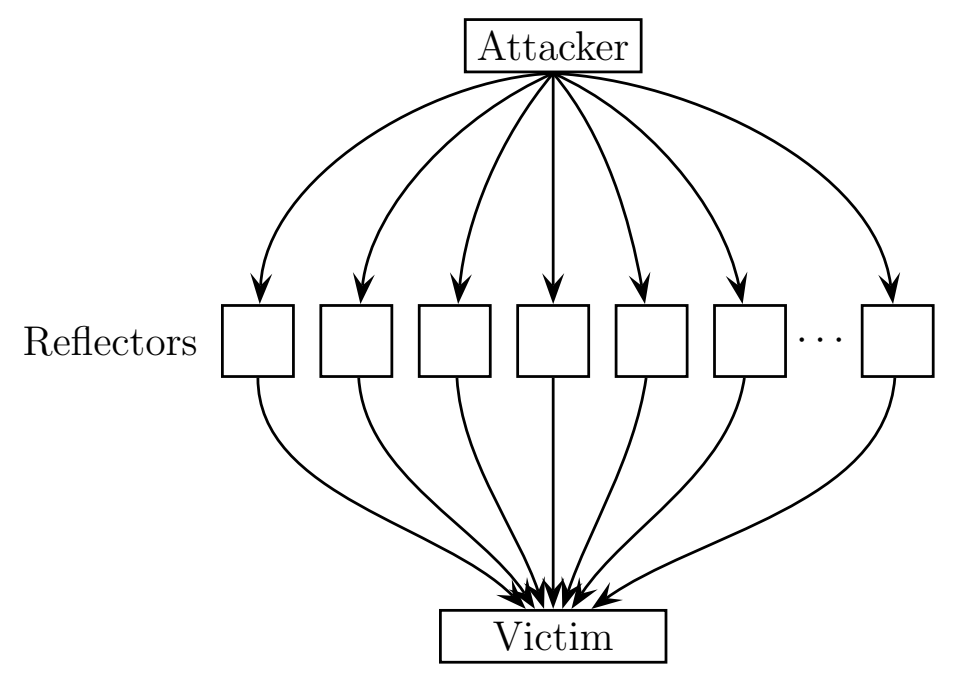

Figure 2.2: DDoS attack network hierarchy.

\subsection{Defense Mechanisms Against DDoS Attacks}

In this section we summarize some of the available defense mechanisms against DDoS attacks. There is currently no comprehensive method to protect against all known forms of DDoS attacks. In addition, many derivative DDoS attacks are continually being developed by attackers to circumvent new countermeasures employed [74]. Chang [13] identifies three lines of defense against DDoS attacks with reference to the start and end of the attack: attack prevention and preemption, attack detection and filtering, and attack source traceback and identification. Attack prevention and preemption includes taking security measures that would protect a host against master and agent implants [13]. 
A large number of IP traceback schemes have been proposed for attack source traceback and identification $[6,10,26,42,46,49,72,73]$. A majority of these schemes require intermediate routers to encode their identities in the packet so that the packet's path can be reconstructed when necessary. For instance, Savage et al. [70] proposed a probabilistic packet marking scheme for IP traceback. Conceptually, the approach works as follows. Three additional fields are stored in each packets: one for the start address, one for the end address, and the third one for distance. If the start address and the end address fields of a packet are marked, then the edge (start address, end address) belongs to the path that the packet traversed. (Notice that the start and end addresses are different from the source and destination addresses.) A packet gets marked by each router it encounters with a preset probability. When a router decides to mark a packet, it writes its own address in the start field and writes a zero in the distance field. If the distance field is already zero, it means the packet was marked by the previous router. In this case, the router writes its own address in the end field and increments the distance field. On the other hand, if the router does not mark the packet, it increments the distance field. When a packet arrives at the victim, its distance field represents the number of hops since the edge (start address, end address) was sampled. Once the victim has received a representative number of marked packets, it can reconstruct the path by ordering the edges according to the number of hops, and thus, identify the attacker.

Burch and Cheswick [10] proposed a route inference approach to IP traceback. The method works by systematically flooding candidate network links and observing for variations in the flow of attack packets due to the restricted bandwidth. The idea is that if the flooded link is on the attackers path, then there must be a significant drop in the flow of the attack packets. The process starts by constructing a map of routes from the victim to every network. Then starting with the router closest to the victim, a brief burst of load is applied to each link attached to the router, each time observing the intensity of the attacking stream at the victim. If the stream is altered when a link is loaded, then that link is probably on the attack path. The process works back through the network router by router, pruning branches that do 
not perturb the attack stream.

In general, IP traceback techniques are not able to mitigate the effects of DDoS attacks while the attack is going on; they are usually after-the-fact responses to DDoS attacks $[13,77]$.

A number of filtering mechanisms have been proposed for attack detection and filtering. Ferguson and Senie [29] proposed the use of border routers to filter out outgoing packets with spoofed IP addresses outside of the internal network. Although this filtering mechanism prohibits the attacker within the internal network from launching DDoS attacks with forged source addresses that do not conform to the internal network, it cannot stop attacks whose forged source address are from within the network [29, 69].

Peng et al. [66] proposed a History-based IP Filtering (HIF) as a defense against DDoS attacks. The approach is based on the assumption that DDoS attack traffic uses randomly spoofed source addresses to disguise their true identities. A record of all the IP addresses of the previous successful connections is compiled into an IP Address Database (IAD). When the network experiences a high level of congestion, packets whose source addresses do not appear in the IAD can be discarded. The HIF is only activated when a high level of network activity is detected.

Park and Lee [63] introduced route-based packet filters to defend against DDoS attacks. Route-based packet filters use routing information to determine if a packet arriving at a router is valid with respect to its purported source/destination addresses. We discuss route-based packet filters in details in Section 2.3. Other approaches to mitigating DDoS attacks include [66], Network Measurement Systems [82], Window-based Packet Filtering [39], and Hop-count Filtering [41].

2.3 Defending Against Flooding-based DDoS Attacks with Route-based Packet Filters

In this section we discuss the use of distributed route-based filters, first proposed by Park and Lee [63], to mitigate DDoS attacks. It is assumed that a filtered node 
cannot forge its origin address. Route-based filters are placed on a subset of nodes on a network and work by analyzing traffic in the network to verify whether the purported origin address on packets entering a filtered node can be legitimate. If a filter can determine that a packet has a forged address, it drops the packet from the network, and otherwise it lets the packet proceed on its path. A packet having a valid origin address should never be dropped by a filter; this requirement is called safety [4].

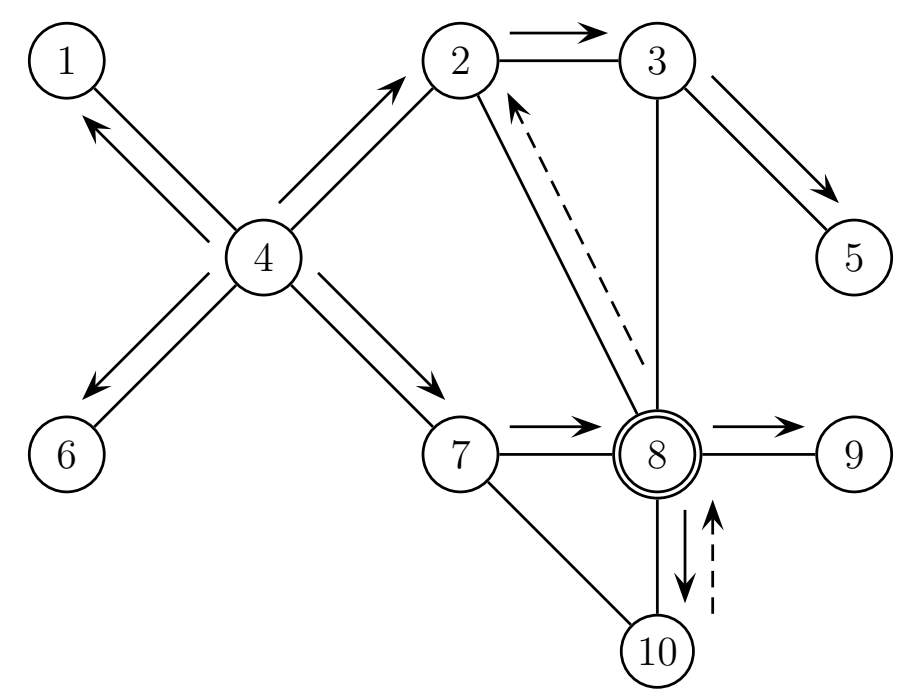

$\longrightarrow$ Node 4 routing to all other nodes

$-\rightarrow \quad$ Attack from node 10 with forged node 4 source address

Figure 2.3: A route-based filter placed on node 8 will stop an attack on node 2 from node 10 with forged node 4 address.

Figure 2.3 illustrates how route-based packet filters function. Solid arrows in the figure show the routing of node 4 to all other nodes. Each edge $(i, j)$ in the graph is bidirectional, representing both $\operatorname{arcs}(i, j)$ and $(j, i)$. A route-based filter place on node 8 will stop an attack on node 2 from node 10 with purported source address of node 4 (dashed arrows) since node 4 never uses the arc $(10,8)$. (A filter on node 2 will also stop this attack because node 4 never uses the arc $(8,2)$. Also, a filter placed on node 10 itself would stop any attack from node 10.) 
We present some notation used in [63]. An Internet Autonomous System (AS) topology was represented by an undirected graph $G=(V, E)$. (An AS is a connected group of one or more IP prefixes run by one or more network operators which has a single and clearly defined routing policy [36].) A set of all loop-free paths from $u$ to $v$ was denoted by $\mathcal{L}(u, v)$. A routing algorithm chooses a subset $R(u, v) \subseteq \mathcal{L}(u, v)$ for routing packets from $u$ to $v$. An IP packet $M(s, t)$ with source IP address $s$ and destination IP address $t$ was routed through the network according to $R(s, t)$. The notation $e \in R(s, t)$ was used to denote that link $e$ is on some path belonging to $R(s, t)$.

Park and Lee [63] identified two types of route-based filters depending on how the verification of packets' authenticity was performed. A maximal filter references both the packet's inscribed origin and destination address to ascertain its authenticity based on the link on which it arrived. The maximal filter function was formally defined as

$$
F_{e}^{m}(s, t)= \begin{cases}0 & \text { if } e \in R(s, t) \\ 1 & \text { otherwise. }\end{cases}
$$

A semi-maximal filter on the other hand only references the packet's inscribed purported origin address to determine its validity based on the arc it arrived on. This mechanism was formally stated as

$$
F_{e}^{s}(s, t)= \begin{cases}0 & \text { if } e \in R(s, v) \text { for some } v \in V \\ 1 & \text { otherwise }\end{cases}
$$

While a maximal filter performs a stronger test than a semi-maximal filter, it requires an order $O\left(n^{2}\right)$ lookup table as opposed to the $O(n)$ lookup table for the semi-maximal filter. Figure 2.4 illustrates the difference in the filtering capabilities of a maximal filter versus a semi-maximal filter. If node 7 attacks node 2 (along dashed arrows) using a purported node 4 source address, then a maximal filter placed on node 8 will stop such an attack since node 4 does not route to node 2 through the arc $(7,8)$. On the other hand, a semi-maximal filter on node 8 cannot 
stop the depicted attack since node 4 does use the arc $(7,8)$ when routing to at least one of its destinations (nodes 8,9 and 10). Also, putting either a maximal or semi-maximal filter on node 2 will stop the attack.



$\longrightarrow \quad$ Node 4 routing to all other nodes

$-\rightarrow \quad$ Attack from node 7 with forged node 4 source address

Figure 2.4: Maximal versus semi-maximal filtering example.

A key performance variable identified in [63] was the selection of nodes on the network on which to place filters. Park and Lee [63] experimented with two strategies for choosing nodes to filters. The first strategy involved randomly picking nodes uniformly from the entire node set in a network until a target number of nodes had been picked. The second strategy involved picking a subset of nodes that formed a vertex cover. Since finding a minimal vertex cover (VC) in a graph is an NP-hard problem [31], they employed a heuristic to find a VC. Results showed that, other parameters being equal, the VC filter placement strategy outperformed the random strategy by far. 


\subsection{The Distributed Packet Filter Placement Problem}

The problem of route-based filter placement was formally studied in [4]. The authors refer to this problem as the Minimum Filter Placement Problem (MFPP) and define it as an optimization problem that seeks to identify a minimum cardinality set of nodes on a network, such that deploying filter at these nodes ensures that any packet with a spoofed source address is dropped by one of the deployed filters. They show that the MFPP is strongly NP-hard by transforming an arbitrary instance of a vertex cover problem into an instance of the MFPP. We provide a sketch of the NP-hard proof from [4]. For continuity, we start with some notation used in the study.

A communication network is represented by a directed graph $G=(N, A)$ in which $N$ such that $|N| \geq 3$ is the set of nodes and $A$ is a set of directed loop-free

arcs. A communication set $C \subseteq N \times N-\left\{\cup_{i \in N}(i, i)\right\}$ is a set of all node pairs such that $(u, v) \in C$ if and only if $u$ and send packets to $v$. A communication set is said to be complete if $(u, v) \in C$ for all node pairs $u \in N$ and $v \in N \backslash\{u\}$, and the corresponding network is said to have complete routing.

The MFPP was first possed as a decision problem (FILTER) to aid in the NPcomplete proof.

FILTER: Given a connected network $G=(N, A)$ with $|N| \geq 3$, a communication set $C$, a routing policy $R$, where $|R(o, d)|$ is polynomially bounded for each $(o, d) \in C$, and an integer $1 \leq k \leq|N|$, does there exist a filter placement $F \subset N,|F| \leq k$ that achieves perfect security?

The Vertex Cover problem is defined as Garey and Johnson [31]:

VERTEX COVER: Given an undirected, connected graph $G=(V, E)$ with $|V| \geq 3$, and an integer $1 \leq c \leq|V|$, does there exist a subset $S$ of $c$ or fewer nodes in $V$ such that if an edge $(u, v) \in E$, then at least one of $u$ and $v$ belongs to $S$ ?

The authors showed that the problem FILTER is in the class NP (we omit this 
part). They transform an arbitrary instance of VERTEX COVER to an instance of FILTER as follows. They set $N:=V$ and $\operatorname{add} \operatorname{arcs}(u, v)$ and $(v, u)$ to $A$ if and only if $(u, v) \in E$, and they set $k$ equal to $c$, also, $C$ equals $A$. The routing $R$ was defined only as a single-arc route that connects adjacent nodes (thus, $|R(u, v)|=1$ for every $(u, v) \in C)$. From this transformation, the authors show that a VERTEX COVER instance is a yes instance if and only if the corresponding FILTER instance is a yes instance, thus proving that the FILTER problem is NP-hard in the strong sense (since no numerical data was used in the proof).

The MFPP was formulated a 0/1 integer program (2.1). The set $N_{\text {sod }}$ in constraint set (2.1b) was defined as the set of all nodes $i$ such that a filter at node $i$ would drop any packet originating from $o$ with destination $d$ having forged address $s$. The MFPP can then be formulated as:

$$
\begin{aligned}
\operatorname{minimize} & \sum_{i \in N} y_{i} \\
\text { s.t. } & \sum_{i \in N_{\text {sod }}} y_{i} \geq 1 \forall(o, d) \in C, s \neq o:(s, d) \in C \\
& y_{i} \in\{0,1\} \quad \forall i \in N .
\end{aligned}
$$

The objective function (2.1a) minimizes the number of filters placed in the network, while constraints (2.1b) eliminate the possibility of any node $o$ forging any address $s$ while sending to any node $d$.

The study outlined some conditions necessary for a filter placement to be feasible to the MFPP. In addition, a necessary and sufficient condition was given that served as a basis for an optimal algorithm for solving the MFPP with complete routing. We list these conditions here without proof and refer the reader to [4] for proofs.

Necessary Condition 1. A solution $F$ to the MFPP with complete routing must also be a feasible vertex cover on the graph $G=(N, E)$, where $(i, j) \in E$ if and only if either $(i, j) \in A$ or $(j, i) \in A$.

Necessary Condition 2. For any feasible solution solution $F$ to the MFPP, if node 
$v \in N$ is not an endpoint, then $v \in F$. (An endpoint was defined as a node that never serves as an intermediate point in the routing any packet.)

Necessary and Sufficient Condition: If $D$ is a set of non-endpoints in $G$ and $S$ is an optimal solution to the Minimum Vertex Cover Problem (MVCP) over the endpoint network $\tilde{G}$, then filtering the nodes $F=D \cup S$ results in an optimal solution to the MFPP, regardless of whether maximal or semi-maximal filters are used. (Here, MVCP is defined to be the optimization variant of VERTEX COVER in which the objective is to minimize the cardinality of the vertex cover for some given graph, and $\tilde{G}$ is the graph induced by the endpoints.)

The necessary and sufficient condition leads to an optimal algorithm for solving the MFPP with complete routing. The algorithm starts by placing filters on all non-endpoints. Then the MVCP is solved on the resulting endpoint graph $\tilde{G}$. Since $\tilde{G}$ is typically disconnected, this results in a decomposition algorithm in which small MVCP are solved independently on the components of $\tilde{G}$. The decomposition presents an opportunity for parallelism while the relationship between the MFPP and the MVCP permits a direct application of the rich theoretical and practical results for the vertex cover problem to the MFPP.

As a consequence of the algorithm suggested by the necessary and sufficient condition, a class of polynomially solvable MFPPs were identified. These are problems with complete routing that yield no vertex cover problem phase, or yield a set of vertex cover problems that are polynomially solvable. They include tree, cycle, and bipartite network topologies. 


\section{CHAPTER 3}

\section{Mixed-Integer Programming Model and a Greedy Vertex Cover Heuristic for the GMFPP}

In this chapter we formally define the Generalized Filter Placement Problem (GMFPP). We model the problem as a mixed-integer program and illustrate its performance on randomly generated networks. Next, we extend the greedy vertex cover heuristic given in [4] to the GMFPP and evaluate its effectiveness against the mixed-integer programming model.

\subsection{The GMFPP: Definition and Notation.}

We follow the notation given in [4] to define the filter placement problem. We define a communication set $C \subseteq N \times N$ such that $(u, v) \in C$ if and only if node $u \in N$ is permitted to send packets to node $v \in N$. We say that $C$ is complete if $(u, v) \in C$ for all node pairs $u \in N$ and $v \in N \backslash\{u\}$. Packets sent to any node $d \in N$ with purported address $o$ such that $(o, d) \notin C$ will automatically be dropped by the network without the need for a filter. A routing policy that ignores packets with $(o, d)$ pairs that do not belong to $C$ is referred to as a "trivial filter." Next, for all node pairs $(o, d) \in C$, define $R(o, d)$ as the set of all paths that a packet from $o$ to $d$ may take under the given routing policy. We denote the set of nodes in a path $p$ by $N(p)$ and the set of arcs in $p$ by $A(p)$. We assume that packet attributes (e.g., the addresses of the purported origin node and destination node) can only be set by the sending node, and hence once the packet is sent, none of its attributes can be changed by intermediate nodes.

Given our filtering mechanism, we define the set of nodes $G_{\text {sod }}$ such that $v \in G_{\text {sod }}$ if and only if a filter placed on node $v$ will drop packets from $s \in N$ to $d \in N \backslash\{s\}$ with a forged origin address of $o \in N \backslash\{d, s\}$. (Note that these sets depend on 
whether maximal or semi-maximal filters are used.) Observe that $s \in G_{\text {sod }}$ for all possible choices of $s, o$, and $d$, since a filtered node cannot attack other nodes. To compute these $G$-sets, we first identify all arcs that node $O$ uses in any of its paths to other nodes. Assume that $|R(u, v)| \leq q$, for some integer $q, \forall(u, v) \in C$. Then identifying these arcs for each of the nodes requires $O\left(q n^{2}\right)$ computations. Next we create each set $G_{\text {sod }}$ by comparing each path in the set $R(o, d)$ to the set of arcs used by node $o$. If a path $p^{\prime} \in R(s, d)$ has an arc $(i, j)$ that is never used by node $o$, then we add the node $j$ to the set $G_{\text {sod }}$. The complexity for computing $G_{\text {sod }}$ is thus $O(q n)$ and so the complexity of computing all $G_{\text {sod }}$ sets is $O\left(q n^{4}\right)$.

For a given filter configuration $F$, we define $N_{o d}(F)$ as the set of all nodes $v \in N$ that can successfully send packets to a node $d \in N$ with forged origin address $o \in N$ without being filtered (by definition, $o \notin N_{o d}(F)$ and $d \notin N_{o d}(F)$ ). We refer to the cardinality of the set $N_{o d}(F)$ as the traceback number for the address/victim pair $(o, d)$ and we denote it by $\beta$. If node $d$ is under attack from purported address $o$, it can investigate these $\beta$ candidates one-by-one to ascertain which node is attacking. We define the traceback limit $b$ as the maximum allowable traceback number over all address/victim pairs $(o, d) \in C$. For a given traceback limit $b \geq 0$, the GMFPP identifies a minimum cardinality subset of nodes $F$ on the network such that placing filters on $F$ ensures that $\left|N_{o d}(F)\right| \leq b$ for all $(o, d) \in C$. Observe that if $b \geq n-2$, the optimal solution is $F=\emptyset$ since $\left|N_{o d}(F)\right| \leq b$ is vacuously satisfied for all address/victim pairs $(o, d) \in C$. Hence, we only consider $b$ in the range $0 \leq b \leq n-3$, where $b=0$ represents the perfect security case.

\subsection{Mixed-Integer Programming Model}

In this section we formulate the fundamental mixed-integer programming model for the GMFPP. We define binary variables $y_{i}$ for each node $i \in N$. The mixed-integer program will set $y_{i}=1$ if a filter is placed on node $i$ in the solution, and $y_{i}=0$ otherwise. We also define a set of auxiliary variables $w_{\text {sod }}$ for each possible attack on node $d \in N$ by node $s \in N$ with forged origin address $o \in N$, where $s \neq o$, 
$(s, d) \in C$ and $(o, d) \in C$. The variable $w_{\text {sod }}=1$ if an attack on $d$ by $s$ using forged origin address $o$ will not be stopped by the set of deployed filters, else, $w_{\text {sod }}=0$. We formulate the GMFPP as:

$$
\begin{aligned}
\operatorname{minimize} & \sum_{i \in N} y_{i} \\
\text { s.t. } & \sum_{s:(s, d) \in C, s \neq o} w_{\text {sod }} \leq b, \forall(o, d) \in C \\
& w_{\text {sod }}+\sum_{i \in G_{\text {sod }}} y_{i} \geq 1 \quad \forall(o, d) \in C, \forall(s, d) \in C: s \neq o \\
& y_{i} \text { binary } \forall i \in N \\
& 0 \leq w_{\text {sod }} \leq 1 \forall s \in N, \forall o \in N, \forall d \in N:(s, d) \in C,(o, d) \in C, s \neq o .
\end{aligned}
$$

The objective function (3.1a) minimizes the total number of filters placed in the network. Constraints (3.1b) limit the number of nodes $s$ that can successfully send packets to node $d$ with purported address $o \neq s$ to at most the traceback limit $b$. Constraints (3.1c) state that node $s$ must be allowed to attack node $d$ by sending packets with purported address $o$, unless a filter is placed on one of the nodes in $G_{\text {sod }}$ that prevents such an attack. Finally, (3.1d) and (3.1e) state the logical restrictions on the variables $y$ and $w$. Observe that we define the $w$-variables as continuous variables instead of binary in (3.1e) because there exists a solution to this relaxed problem in which all $w$-variables are binary. The argument to verify this claim starts by observing that any optimal solution to the relaxed problem still requires binary $y$-variables. Consider any optimal solution having some fractional-valued variable $\hat{w}_{s o d}=f(0<f<1)$. Then according to constraints (3.1c), we must have $y_{i}=1$ for some $i \in G_{\text {sod }}$. Hence, revising the variable $\hat{w}_{\text {sod }}$ to equal 0 instead of $f$ retains feasibility with respect to (3.1c). Furthermore, decreasing this variable value does not affect its feasibility with respect to (3.1b). Since the objective function (3.1a) does not involve any $w$-variables, this revised solution must also be optimal. 
The mixed-integer programming formulation given in (3.1) must yield an optimal objective function value and set of filter placements. However, it is possible that some $w_{\text {sod }}$-variable is positive in an optimal solution for a node $s$ that actually cannot attack $d$ using forged address $o$. To see this, observe that if $w_{s o d}=0$ for some $(s, o, d)$ node triple defined for (3.1c), then $\sum_{i \in G_{s o d}} y_{i} \geq 1$ necessarily, and thus $s$ cannot attack $d$ using address $o$. However, examining the converse of this statement, it is possible that even though $\sum_{i \in G_{s o d}} y_{i} \geq 1, w_{\text {sod }}>0$ is still possible. However, this situation only occurs if the traceback constraint for node pair $(o, d)$ is not a binding constraint (i.e., the actual number of nodes that can attack $d$ with forged address $o$ is strictly less than $b$ ), which permits the optimization process to arbitrarily set $w_{\text {sod }}$ to any value in the range $[0,1]$.

We can address this situation, if desired, by modifying the objective function (3.1a) to include a term that would penalize the number of positive $w$-variables as a secondary consideration. The objective function would then be given as:

$$
\operatorname{minimize} \sum_{i \in N} M y_{i}+\sum_{\text {all }(s, o, d) \text { triples }} w_{s o d},
$$

where $M$ is a number greater than the total number of $(s, o, d)$ sets (e.g., $M=$ $n(n-1)(n-2)+1$ is sufficiently large). This would force the model to ignore any solution that uses more filters than absolutely necessary, due to the given preemptive weight of $M$ on the number of filters selected. However, given this objective, no $w_{\text {sod }}{ }^{-}$ variable will be set equal to 1 at optimality unless an attack prescribed by this triple can indeed occur (or else, a better solution would exist in which $w_{\text {sod }}=0$ ). Hence, employing (3.2) as our objective function serves to define $w_{\text {sod }}=1$ if and only if an attack can occur from $s$ to $d$ using address $o$. However, such an objective function might also require the mixed-integer program to exert even more computational effort, since an additional "tie-breaking" requirement is incorporated into the model. 


\subsection{The Extended Vertex Cover Greedy Heuristic}

We now describe a heuristic that estimates the solution of the GMFPP in polynomial time. The heuristic is based on a greedy vertex cover heuristic that was described in [4]. A vertex cover on a graph is a set of nodes $C$ such that each edge in the graph is incident to at least one node in $C$. Finding a minimum vertex cover is well-known to be NP-hard [31].

The heuristic that we describe below can be can be viewed as a two-phase algorithm, in which the first phase establishes filters on nodes according to a classical greedy vertex cover heuristic until either $|N(u, v)| \leq b$ for all $(u, v) \in C$, or until a vertex cover is established. In the latter case, a second phase is examined in which filters are greedily placed on additional nodes until a feasible solution is determined for the problem. The algorithm is given as follows.

Step 1. The algorithm is initialized by setting the solution $F=\emptyset$. Convert $A$ into an undirected graph by including a single edge $(i, j)$ in $A$ if and only if either $(i, j)$ or $(j, i)$ was previously in $A$. For each $i \in N$, set $d_{i}$ equal to the degree of node $i$ (i.e., the number of arcs incident to node $i$ ). Establish a list $L$ of node pairs in the communication set $C$.

Step 2. If $L$ is empty, go to Step 8. Otherwise, choose the next pair $(u, v) \in L$, and execute Step 3.

Step 3. If $\left|N_{u v}\right|>b$, execute Step 4. Otherwise, remove $(u, v)$ from $L$ and return to Step 2.

Step 4. Identify node $i \in \operatorname{argmax}_{k \in N}\left\{d_{k}\right\}$. If $d_{i}=0$, go to Step 5, and otherwise, go to Step 6.

Step 5. Find a node $\ell \notin F$ such that placing a filter on $\ell$ will stop the most number of attacks on $v$ with purported address $u$. Add $\ell$ to $F$. Go to Step 7 .

Step 6. Add node $i$ to $F$. Reduce $d_{i}$ and $d_{j}$ by 1 for all $j$ such that $(i, j) \in A$ or $(j, i) \in A$. Go to Step 7 .

Step 7. Update $N_{u v}$, and return to Step 3.

Step 8. Return $F$ as the heuristically generated feasible solution to the GMFPP. 
Note that the inner loop of the algorithm, consisting of Steps 4 - 7, must be executed no more than $n$ times, since a filter is located on the network at each of these iterations. The most time-consuming step in this inner loop is encountered in Step 5, which takes $O\left(n^{2}\right)$ operations. (The algorithm must examine all possible attacking origins, and compare the nodes on the paths from those destinations to $v$ with the path from $u$ to $v$, as done in the calculation of the $G$ sets mentioned for the mixed-integer programming model.) Hence, the overall complexity for this heuristic is $O\left(n^{3}\right)$.

\subsection{Computational Results}

In this section, we present computational results for both the mixed-integer programming model and the heuristic algorithm. In particular, we wish to obtain initial results regarding the computational time of the mixed-integer programming algorithm, and the typical degree of suboptimality encountered due to using heuristic versus optimal solutions.

We first describe the random generation of three sets of problem instances, which we solve in this section by the methods presented earlier. For each instance, we generate a graph of a given size and density. An edge is placed randomly between each pair of nodes $(i, j)$ with probability given by the specified edge density. The first set of instances was generated with 15 nodes and an edge density of 0.4. The second set of instances was generated with 20 nodes and an edge density 0.4 , and lastly, the third set of instances contained 30 nodes and an edge density of 0.2 . We refer to these sets of instances as "small", "medium", and "large" instance sets, respectively. For each instance set, 10 instances were generated, for a total of 30 instances.

In all the instances, we assume complete communication (i.e., each node can send packets to every other node). The routing policy $R(u, v)$ for each pair of nodes $u, v \in N$ returns a single shortest path from node $u$ to node $v$ (assuming equal weights on the edges) for all communication pairs. 
As a visual illustration of the differences possible in using exact versus heuristic solutions, we solved a 13-node problem with $b=3$ using both methods. Figure 3.1 shows this network topology, in which every node communicates with all other nodes along shortest paths. A minimum cardinality set of nodes that must be filtered is shown as the set of double-circled nodes in the figure. The solution obtained from the mixed-integer programming model shows that the minimum number of filters required is five.



Figure 3.1: Mixed-integer Programming Solution, with $b=3$.

We then solved the same problem using the heuristic algorithm, whose solution is displayed in Fig. 3.2. Note that the heuristic solution required two more filters than the optimal solution from the mixed-integer program, which is a significant increase for a problem of this size.

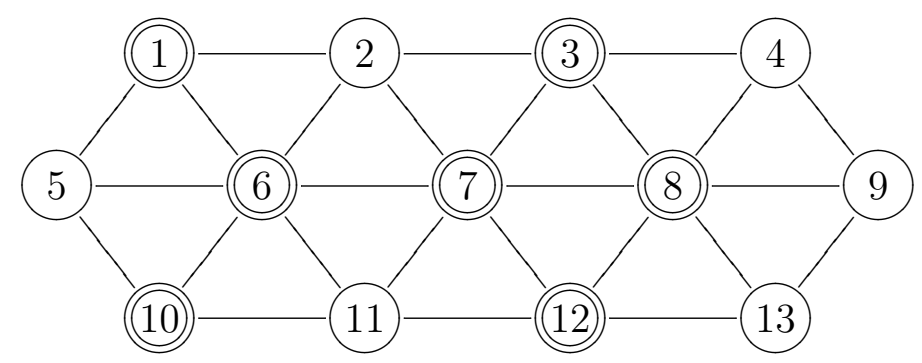

Figure 3.2: Heuristic Solution, with $b=3$.

The primary issue regarding the mixed-integer programming algorithm is its practicality on solving problem instances of varying sizes. We display the average execution time and standard deviation required for solving (3.1) by CPLEX in Figures 3.3-3.5 for each of the three test sets, varying $b$ from its lower limit to its upper 
limit. Observe the sharp rate of increase in the computational time required to solve these instances as the problem size grows from small (15 nodes) to medium (20 nodes) to large (30 nodes). Hence, without further modification to this algorithm, a heuristic strategy must be employed to solve problems larger than the ones investigated in this preliminary study.

Another important observation regards the peak of the computational times when $b$ is roughly equal to $30 \%$ of $n$. This is due to the fact that few filters are in fact required when $b$ becomes moderately large, and many small subsets of $N$ serve as alternative optimal solutions. (As is typical of most mixed-integer programming problems, the vast majority of computational effort is spent in proving the optimality of a solution obtained very early in the algorithm.) On the other hand, when $b$ is very small, the problem is easier to solve, because several filters are fixed early in a preprocessing stage. The truly difficult problems seem to be those for which $b$ lies in between these values.
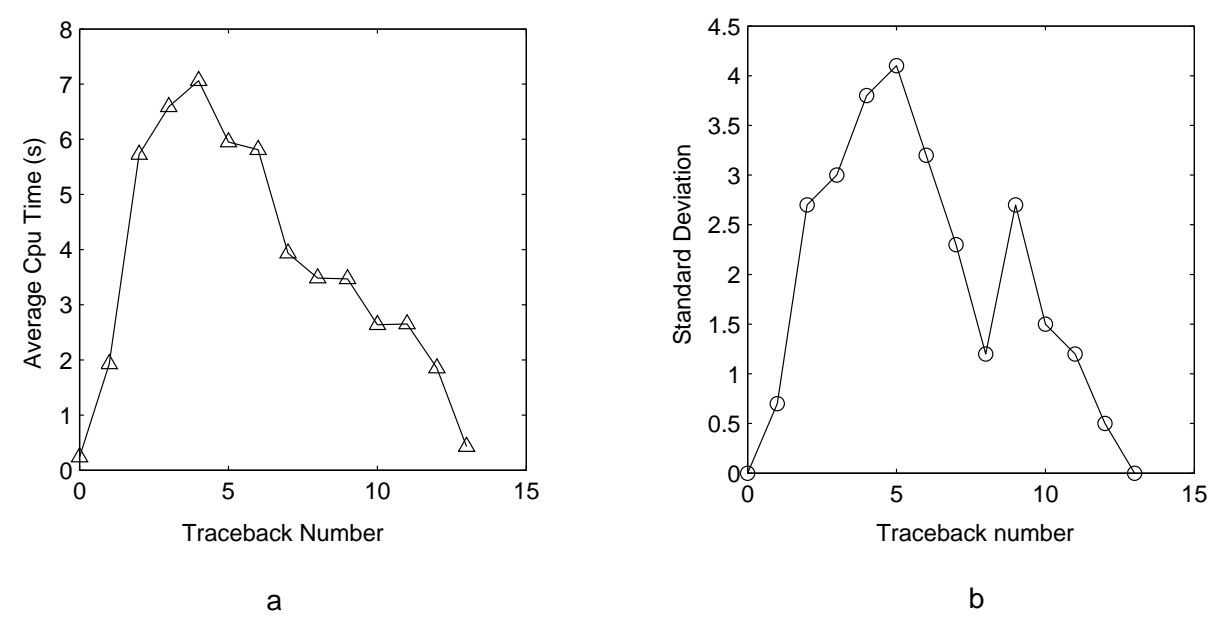

Figure 3.3: Small instance set. (a) average Cpu times vs traceback number. (b) standard deviation of computational times vs traceback number.

Given the motivation for heuristic study, we then briefly examine the quality of heuristic solutions on the problems generated. Tables 4.1-4.3 display the results of our experiments, where the solution obtained by the heuristic is compared against 


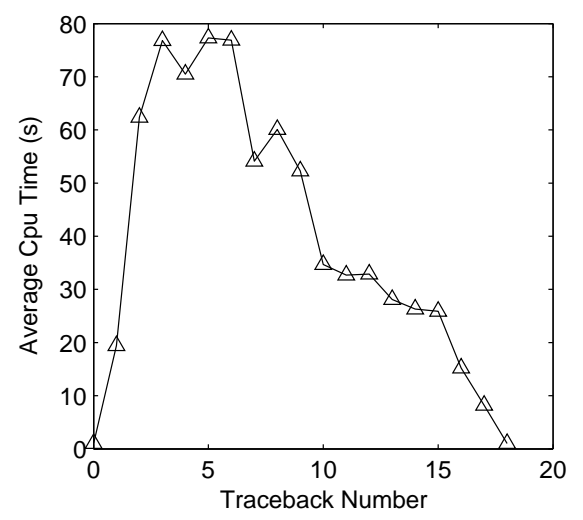

a

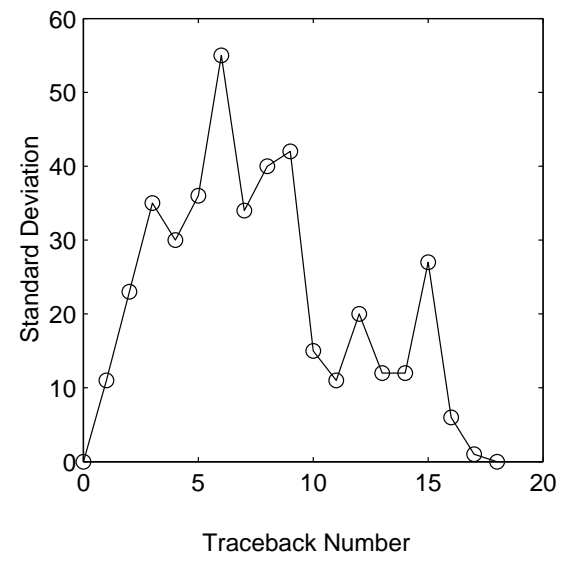

b

Figure 3.4: Medium instance set. (a) average Cpu times vs traceback number. (b) standard deviation of computational times vs traceback number.

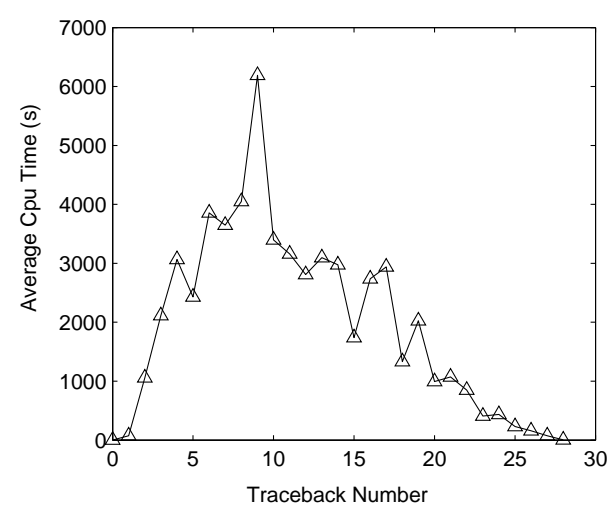

a

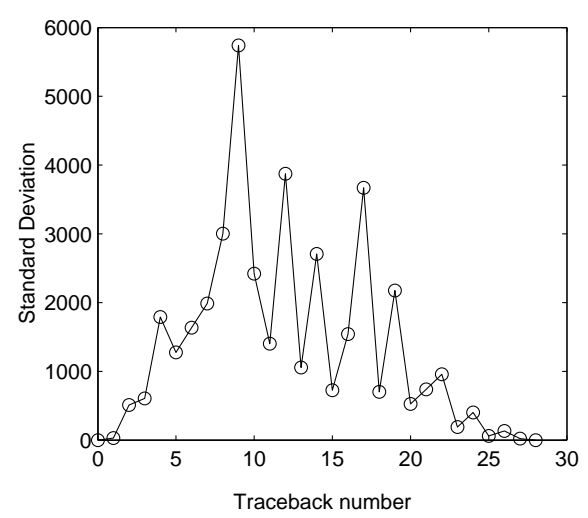

Figure 3.5: Large instance set. (a) average Cpu times vs traceback number. (b) standard deviation of computational times vs traceback number. 
the known optimal solution obtained from our prior experiments with the mixedinteger programming formulation. The column labeled $b$ again displays the traceback numbers for which the problem was run. The columns $z_{i}^{*}, i=1, \ldots, 10$ represent the optimal number of filters for instance $i$ determined by the mixed-integer program. The "suboptimality" columns $q=0,1,2$, and 3 (for the last two cases) indicate the number of instances, out of 10 , for which the heuristic solution was worse than the optimal solution by $q$ filters. (Thus, column 0 is the number of times that the heuristic correctly identified the optimal solution.) The heuristic solution was always within 3 filters of the optimal solution for each instance examined. The execution time for these heuristics is not recorded, since all terminated in under 1 second of CPU time. Observe that the suboptimality of the heuristic tends to become more severe as the problem instances become more difficult for the mixed-integer programs to solve. Indeed, there seems to be a direct correlation between the CPU seconds required for CPLEX to solve instances to optimality and the suboptimality encountered by the heuristic approach.

Table 3.1: Quality of heuristic solutions compared to optimal solutions for the small instance set.

\begin{tabular}{c|cccccccccc||ccc}
\multicolumn{10}{|c||}{ Optimal Number of Filters } & \multicolumn{1}{c|}{ Suboptimality } \\
$b$ & $z_{1}^{*}$ & $z_{2}^{*}$ & $z_{3}^{*}$ & $z_{4}^{*}$ & $z_{5}^{*}$ & $z_{6}^{*}$ & $z_{7}^{*}$ & $z_{8}^{*}$ & $z_{9}^{*}$ & $z_{10}^{*}$ & 0 & 1 & 2 \\
\hline 0 & 11 & 12 & 12 & 10 & 10 & 12 & 13 & 13 & 12 & 11 & 3 & 5 & 2 \\
1 & 8 & 9 & 8 & 8 & 9 & 9 & 9 & 9 & 10 & 9 & 5 & 3 & 2 \\
2 & 7 & 8 & 7 & 6 & 8 & 7 & 8 & 8 & 8 & 7 & 8 & 2 & 0 \\
3 & 6 & 7 & 6 & 6 & 7 & 6 & 7 & 7 & 7 & 7 & 7 & 3 & 0 \\
4 & 5 & 6 & 6 & 5 & 6 & 5 & 6 & 6 & 6 & 6 & 5 & 5 & 0 \\
5 & 5 & 5 & 5 & 5 & 6 & 4 & 5 & 6 & 6 & 5 & 5 & 5 & 0 \\
6 & 5 & 5 & 4 & 3 & 5 & 4 & 5 & 5 & 5 & 4 & 5 & 4 & 1 \\
7 & 4 & 4 & 4 & 3 & 4 & 4 & 4 & 5 & 4 & 4 & 4 & 6 & 0 \\
8 & 4 & 3 & 3 & 3 & 4 & 3 & 4 & 4 & 4 & 3 & 5 & 4 & 1 \\
9 & 3 & 3 & 3 & 3 & 3 & 3 & 3 & 4 & 4 & 3 & 6 & 4 & 0 \\
10 & 3 & 3 & 3 & 2 & 3 & 3 & 3 & 3 & 3 & 3 & 7 & 3 & 0 \\
11 & 2 & 2 & 2 & 2 & 3 & 3 & 2 & 3 & 3 & 2 & 8 & 2 & 0 \\
12 & 2 & 2 & 2 & 2 & 2 & 2 & 2 & 2 & 2 & 2 & 10 & 0 & 0 \\
13 & 0 & 0 & 0 & 0 & 0 & 0 & 0 & 0 & 0 & 0 & 10 & 0 & 0
\end{tabular}


Table 3.2: Quality of heuristic solutions compared to optimal solutions for the medium instance set.

\begin{tabular}{c|cccccccccc||cccc} 
& \multicolumn{10}{|c||}{ Optimal Number of Filters } & \multicolumn{7}{|c}{ Suboptimality } \\
$b$ & $z_{1}^{*}$ & $z_{2}^{*}$ & $z_{3}^{*}$ & $z_{4}^{*}$ & $z_{5}^{*}$ & $z_{6}^{*}$ & $z_{7}^{*}$ & $z_{8}^{*}$ & $z_{9}^{*}$ & $z_{10}^{*}$ & 0 & 1 & 2 & 3 \\
\hline 0 & 20 & 16 & 16 & 16 & 17 & 18 & 17 & 17 & 16 & 15 & 5 & 3 & 0 & 2 \\
1 & 12 & 14 & 13 & 13 & 12 & 13 & 14 & 14 & 13 & 12 & 4 & 4 & 2 & 0 \\
2 & 11 & 12 & 11 & 11 & 11 & 11 & 12 & 12 & 11 & 10 & 3 & 7 & 0 & 0 \\
3 & 10 & 11 & 10 & 10 & 9 & 10 & 11 & 10 & 11 & 9 & 2 & 8 & 0 & 0 \\
4 & 9 & 10 & 9 & 9 & 8 & 8 & 10 & 9 & 10 & 8 & 1 & 6 & 3 & 0 \\
5 & 8 & 9 & 9 & 9 & 7 & 8 & 9 & 9 & 9 & 7 & 1 & 6 & 3 & 0 \\
6 & 7 & 9 & 7 & 8 & 7 & 7 & 8 & 8 & 8 & 6 & 0 & 3 & 6 & 1 \\
7 & 7 & 8 & 7 & 7 & 6 & 6 & 8 & 7 & 7 & 6 & 0 & 5 & 4 & 1 \\
8 & 7 & 7 & 6 & 7 & 6 & 6 & 7 & 6 & 6 & 6 & 1 & 4 & 4 & 1 \\
9 & 6 & 7 & 5 & 6 & 5 & 5 & 6 & 6 & 5 & 5 & 0 & 5 & 4 & 0 \\
10 & 5 & 6 & 5 & 5 & 5 & 5 & 6 & 5 & 5 & 5 & 1 & 4 & 3 & 2 \\
11 & 4 & 5 & 5 & 5 & 4 & 5 & 5 & 5 & 4 & 4 & 1 & 3 & 3 & 3 \\
12 & 4 & 5 & 4 & 5 & 4 & 4 & 5 & 5 & 4 & 4 & 1 & 4 & 3 & 2 \\
13 & 4 & 4 & 4 & 4 & 4 & 4 & 4 & 4 & 4 & 4 & 1 & 4 & 5 & 0 \\
14 & 3 & 4 & 4 & 4 & 4 & 4 & 4 & 4 & 3 & 3 & 1 & 8 & 1 & 0 \\
15 & 3 & 4 & 3 & 3 & 3 & 3 & 4 & 3 & 3 & 3 & 4 & 6 & 0 & 0 \\
16 & 2 & 3 & 3 & 3 & 2 & 3 & 3 & 3 & 3 & 2 & 7 & 3 & 0 & 0 \\
17 & 2 & 2 & 2 & 2 & 2 & 2 & 2 & 2 & 2 & 2 & 10 & 0 & 0 & 0 \\
18 & 0 & 0 & 0 & 0 & 0 & 0 & 0 & 0 & 0 & 0 & 10 & 0 & 0 & 0
\end{tabular}


Table 3.3: Quality of heuristic solutions compared to optimal solutions for the large instance set.

\begin{tabular}{c|cccccccccc||cccc} 
& \multicolumn{7}{|c||}{ Suboptimality } \\
$b$ & $z_{1}^{*}$ & $z_{2}^{*}$ & $z_{3}^{*}$ & $z_{4}^{*}$ & $z_{5}^{*}$ & $z_{6}^{*}$ & $z_{7}^{*}$ & $z_{8}^{*}$ & $z_{9}^{*}$ & $z_{10}^{*}$ & 0 & 1 & 2 & 3 \\
\hline 0 & 29 & 27 & 27 & 29 & 28 & 27 & 29 & 27 & 29 & 28 & 4 & 4 & 2 & 0 \\
1 & 19 & 19 & 18 & 20 & 18 & 19 & 20 & 18 & 18 & 18 & 0 & 7 & 3 & 0 \\
2 & 16 & 17 & 16 & 17 & 16 & 16 & 17 & 15 & 16 & 16 & 2 & 4 & 3 & 1 \\
3 & 14 & 15 & 14 & 15 & 15 & 15 & 15 & 15 & 15 & 15 & 2 & 5 & 3 & 0 \\
4 & 13 & 14 & 13 & 14 & 14 & 14 & 13 & 13 & 13 & 13 & 0 & 8 & 0 & 2 \\
5 & 12 & 13 & 12 & 13 & 12 & 13 & 12 & 12 & 12 & 12 & 1 & 6 & 3 & 0 \\
6 & 12 & 12 & 12 & 12 & 11 & 12 & 12 & 11 & 11 & 12 & 4 & 4 & 2 & 0 \\
7 & 11 & 11 & 11 & 12 & 10 & 11 & 11 & 11 & 11 & 10 & 3 & 3 & 4 & 0 \\
8 & 10 & 10 & 10 & 11 & 10 & 10 & 11 & 10 & 10 & 9 & 2 & 4 & 4 & 0 \\
9 & 9 & 10 & 10 & 10 & 10 & 10 & 10 & 10 & 9 & 9 & 3 & 5 & 2 & 0 \\
10 & 9 & 9 & 9 & 10 & 9 & 9 & 9 & 9 & 9 & 9 & 3 & 5 & 1 & 1 \\
11 & 9 & 9 & 9 & 9 & 8 & 9 & 9 & 9 & 8 & 8 & 3 & 5 & 1 & 1 \\
12 & 8 & 8 & 8 & 8 & 8 & 8 & 9 & 8 & 8 & 8 & 3 & 4 & 2 & 0 \\
13 & 7 & 8 & 8 & 8 & 7 & 8 & 8 & 8 & 8 & 7 & 2 & 6 & 1 & 1 \\
14 & 7 & 7 & 7 & 7 & 7 & 7 & 7 & 8 & 7 & 7 & 6 & 2 & 0 \\
15 & 6 & 7 & 7 & 7 & 6 & 6 & 7 & 7 & 7 & 6 & 0 & 6 & 2 & 1 \\
16 & 6 & 7 & 6 & 7 & 6 & 6 & 7 & 7 & 6 & 6 & 1 & 7 & 2 & 0 \\
17 & 6 & 6 & 6 & 6 & 6 & 6 & 7 & 6 & 6 & 6 & 1 & 7 & 2 & 0 \\
18 & 5 & 6 & 6 & 6 & 5 & 5 & 6 & 6 & 6 & 5 & 1 & 6 & 3 & 0 \\
19 & 5 & 5 & 5 & 6 & 5 & 5 & 6 & 6 & 5 & 5 & 3 & 4 & 3 & 0 \\
20 & 5 & 5 & 5 & 5 & 5 & 5 & 5 & 5 & 5 & 4 & 2 & 6 & 2 & 0 \\
21 & 5 & 5 & 4 & 5 & 4 & 4 & 5 & 5 & 5 & 4 & 2 & 6 & 2 & 0 \\
22 & 4 & 5 & 4 & 4 & 4 & 4 & 4 & 4 & 4 & 3 & 5 & 4 & 0 \\
23 & 4 & 4 & 4 & 4 & 3 & 4 & 4 & 4 & 4 & 3 & 3 & 4 & 3 & 0 \\
24 & 3 & 3 & 3 & 3 & 3 & 3 & 3 & 3 & 4 & 3 & 7 & 2 & 0 \\
25 & 3 & 3 & 3 & 3 & 3 & 3 & 3 & 3 & 3 & 3 & 6 & 0 & 0 \\
26 & 2 & 2 & 2 & 2 & 2 & 2 & 2 & 2 & 3 & 2 & 2 & 8 & 0 & 0 \\
27 & 2 & 1 & 2 & 2 & 1 & 2 & 2 & 2 & 2 & 2 & 8 & 2 & 0 & 0 \\
28 & 0 & 0 & 0 & 0 & 0 & 0 & 0 & 0 & 0 & 0 & 10 & 0 & 0 & 0
\end{tabular}




\subsection{Summary}

In this chapter we stated the Generalized Minimum Filter Placement Problem and presented a mixed-integer programming model to solve it to optimality. We also presented an $O\left(n^{3}\right)$ heuristic for this problem based on a greedy vertex cover heuristic. Our preliminary studies on this problem indicate that solving the mixed-integer programming model will not be practical on larger instances (say, $n \geq 40$ ), unless some significant work is performed in strengthening the linear programming relaxation to the problem or improving the overall algorithmic approach to solving this model. 


\section{CHAPTER 4}

\section{The GMFPP on Special Network Topologies.}

In this chapter, we identify a set of special network topologies on which the filter placement problem is solvable in polynomial time. We focus our attention on the development of a dynamic programming algorithm for solving the GMFPP on tree networks. Finally we evaluate the performance of the dynamic programming algorithm against a mixed-integer programming model on randomly generated tree networks at varied levels of security.

Before proceeding to discuss special network structures that admit polynomialtime solvability, we first make some key observations regarding solutions to the GMFPP.

Lemma 1. A filter placed on a leaf node of $G$ (i.e., a node with degree one) cannot stop attacks directed at the leaf node.

Proof: Let $\ell$ denote some leaf node of $G$, adjacent to node $k$. All packets coming to node $\ell$ use the link $(k, \ell)$, regardless of whether the origin address is forged or not. Therefore, a filter placed on node $\ell$ is unable to distinguish attacks from legitimate packets.

Lemma 2. If $F^{*}$ is an optimal solution to the GMFPP, then the nodes adjacent to filtered leaf nodes must be unfiltered.

Proof: If $\left|F^{*}\right|=0$, this result clearly holds true. Else, $\left|F^{*}\right|>0$, suppose by contradiction that a filtered node $k$ is adjacent to a filtered leaf node $\ell$. Now, consider a solution in which we remove a filter from node $\ell$. Since $\ell$ is the only node that routes through the link $(\ell, k)$, the filter placed at node $k$ will stop any attacks coming from $\ell$. From Lemma 1, we know that the removal of a filter from a leaf node will only affect attacks coming from that node. Therefore, if both nodes $k$ and 
$\ell$ are filtered, we can remove a filter from $\ell$ without violating the traceback limit, contradicting the optimality of $F^{*}$.

Proposition 1. There exists an optimal solution to the GMFPP in which no leaf node is filtered.

Proof: Let $\bar{F}$ be an optimal solution with $|\bar{F}| \geq 1$. Consider a leaf node $\ell$ and its adjacent node $k$. By Lemma $2, \bar{F}$ does not include both $\ell$ and $k$. If $\ell$ belongs to $\bar{F}$, we construct an alternative optimal solution $F^{*}$ that moves the filter on $\ell$ to node $k$. By Lemmas 1 and 2, such a filter movement does not violate the traceback limit. Repeating this step for all leaf nodes results in an alternative optimal solution for which no filter exists at a leaf node.

Proposition 1 enables us to search a smaller solution space by disregarding all solutions that filter leaf nodes.

\subsection{Structures That Admit Closed-Form Solutions}

We discuss simple network topologies that have closed-form solutions in this section. The first topology we consider is a chain topology. A chain topology is a connected network in which all nodes have degree two except for the two end nodes that have degree one. Given such a network with $n$ nodes and a given traceback limit $b$, the minimum number of filters required to achieve the desired level of security is given by $|F|=n-b-2$. To determine the most vulnerable $(o, d)$ pair, consider a chain with nodes labeled $1, \ldots, \ell$ where 1 and $\ell$ are the leaf nodes. For a given $(o, d)$ pair $(i, j)$, assuming without loss of generality that $i<j$, we observe that any unfiltered node $k, i<k<j$, can successfully send packets to node $j$ with purported address $i$ regardless of the filter placement in the network. None of the deployed filters can prevent such an attack since packets from node $i$ to $j$ use the link $(k, k+1)$.

An unfiltered node $k$ with $k<i$ cannot attack node $j$ with purported address $i$ if at least one of the nodes $k+1, \ldots, i$ is filtered since $i$ never uses any of the links $(s, s+1), s=k, \ldots, i-1$, in any of its routes. Similarly, an unfiltered node $k$ with $k>j$ can only attack node $j$ with purported address $i$ if none of the nodes 
$j, \ldots, k-1$ is filtered. As the value of $i$ is decreased and the value of $j$ increased, we see that the number of unfiltered nodes that can attack node $j$ with purported origin address $i$ will not decrease for a given filter configuration. Therefore, the most vulnerable $(o, d)$ pair is $(1, \ell)$ (or $(\ell, 1)$ ). Putting $|F|=n-b-2$ filters on any set of non-leaf nodes guarantees that the traceback number for the pair $(1, \ell)$ is $b$.

Another network topology that has a closed form solution is a star network. A star topology on $n$ nodes is a connected network in which all nodes have degree 1 except for one node that has degree $n-1$. In such a network, the minimum number of filters needed to attain a required level of security prescribed by a given traceback limit $b$ is given by $|F|=0$ if $b=n-2$, and by $|F|=1$ if $b<n-2$. This follows from the fact that filtering the node with degree $n-1$ provides perfect security for the network.

The last network structure we consider that has a closed-form solution is a clique. A clique on $n$ nodes is a connected simple graph in which all nodes have degree $n-1$ (i.e., there exists an edge between all distinct pairs of nodes). We assume that each node in a clique communicates with other nodes directly along single-arc paths. For such a communication network topology on $n$ nodes and for a given traceback limit $b$, the minimum number of filters needed to satisfy the required security level is $|F|=n-b-1$, for $b=0, \ldots, n-3$, and $|F|=0$ when $b=n-2$. To verify this assertion, we first observe that no filtered node can be attacked in a clique network with single-arc routing. Only unfiltered nodes are susceptible to attacks, and these attacks can only come from unfiltered nodes. So a most vulnerable $(o, d)$ pair is $(f, u)$ where $f$ is a filtered node and $u$ is an unfiltered node. Therefore, the maximum number of nodes that can attack $u$ with purported address $f$ is $n-|F|-1$, resulting in $|F|=n-b-1$. However, when $|F|=1$, we get a traceback number of $n-2$, which is equivalent to the traceback number when no filters are deployed. Thus, when $b=n-2$, no filters are required. 


\subsection{Tree Networks}

In this section we provide a dynamic programming scheme for solving the GMFPP on tree networks. We first introduce some additional notation used in describing key elements of the dynamic program in Section 4.2.1. We next give the description of the algorithm in Section 4.2.2, and analyze the computational complexity of the algorithm in Section 4.2.3.

\subsubsection{Dynamic Programming Notation}

A tree $G=(N, E)$ is a simple connected graph with $n=|N|$ nodes and $n-1$ edges. Chain and star graphs are special cases of tree networks. While tree networks exhibit some special structures common to chains and stars that we exploit in the following polynomial-time algorithm (namely, that a unique path exits between every pair of nodes), the solution procedure becomes much more complicated for general tree structures.

For this algorithm, we denote a longest path in $G$ by $p_{\ell}$, and define $n_{\ell}$ to be the number of nodes in $p_{\ell}$. We designate the $\left\lceil n_{\ell} / 2\right\rceil^{\text {th }}$ node in the chain as the root node

of the tree $G$, and denote it by $r$. Choosing a root node in this manner minimizes the depth of the tree on which the dynamic programming algorithm operates. We define the level $\ell_{v}$ of node $v \in N$ as the length of the path from $v$ to the root node $r$, thus, $\ell_{r}=0$. By construction, our tree contains $K=\left\lfloor n_{\ell} / 2\right\rfloor+1$ levels. We let $S_{v}$ denote the children of node $v \in N$, which are the nodes at level $\ell_{v}+1$ that are adjacent to node $v$. The node adjacent to $v$ at level $\ell_{v}-1$ is the parent of $v$ and is denoted by $p^{(v)}$. We also define descendants of node $v$ to be the set of nodes $D_{v}$, $D_{v} \supseteq S_{v}$, such that the path from a node $u \in D_{v}$ to the root node $r$ includes node $v$ as an intermediate node.

Define $T_{v}$ to be the subtree induced by the node set $\{v\} \cup D_{v}$, and define $L_{v}$ to be the set of all leaf nodes in $T_{v}$. In Figure $4.1, T_{3}$ contains nodes $\{3,5,6,8,9,10,11\}$, $S_{3}=\{5,6\}, D_{3}=\{5,6,8,9,10,11\}$, and $L_{3}=\{8,9,10,11\}$. 


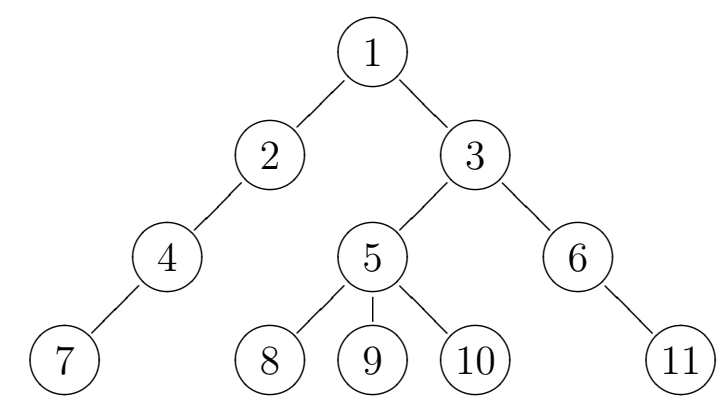

Figure 4.1: An illustration of the tree network topology.

\subsubsection{Dynamic programming algorithm}

The dynamic programming algorithm we describe in this section solves the GMFPP on tree networks by examining subtrees, starting from leaf nodes and progressing in stages towards the root node. At each stage, we consider a node $v$ such that for every $u \in S_{v} \backslash L_{v}$, the subtree $T_{u}$ has already been examined by our procedure. We combine solutions from the subtrees $T_{u}, \forall u \in S_{v}$, to obtain a set of nondominated solutions for the subtree $T_{v}$. Thus, for any given number of filters $n_{f} \in\left\{0, \ldots, r_{v}\right\}$, where $r_{v}=\left|D_{v}\right|-\left|L_{v}\right|+1$ is the minimum number of filters needed to achieve perfect security in the subtree $T_{v}$, we determine how to optimally deploy $n_{f}$ filters in $T_{v}$ to minimize the maximum traceback number in the subtree.

Before describing the dynamic programming algorithm, we first discuss a node aggregating procedure that simplifies the network structure and the computation of the maximum traceback number. Consider the network shown in Figure 4.2a, in which the shaded nodes indicate the placement of filters. We aggregate contiguous unfiltered nodes into single weighted nodes. Weights on the aggregated nodes indicate the number of nodes that were combined to form the aggregated node. Figure $4.2 \mathrm{a}$ shows the sets of contiguous unfiltered nodes to be aggregated enclosed in dotted ellipses. Figure $4.2 \mathrm{~b}$ shows a simplified network after the node aggregation.

Once nodes have been aggregated, the computation of the maximum traceback number for the network is reduced to finding a longest path (in terms of node 




(a) Nodes to be aggregated.

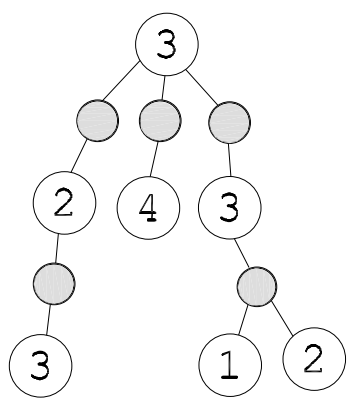

(b) Simplified network after node aggregation.

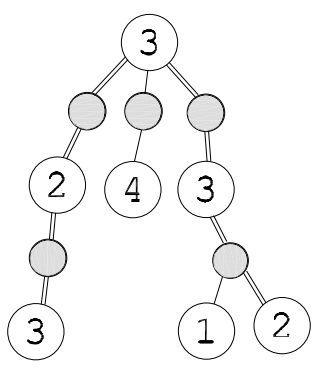

(c) Longest path highlighted with double edges.

Figure 4.2: The process of node aggregation.

weights) in the network. In the network depicted in Figure $4.2 \mathrm{c}$, the longest path is highlighted with double edges. Given the total weight $w$ of a longest path, we compute the maximum traceback number of the network as $w-2$. In our example in Figure 4.2c, $w=13$ and the maximum traceback number is 11 .

When the aggregation procedure is applied to a subtree $T_{v}$, node $v$ itself will either be filtered or it will be part of an aggregated node. In the latter case, we refer to the set of all unfiltered nodes included in the aggregated root node as an open set, denoted by $O_{v}$. If node $v$ is filtered, then the open set is empty.

We denote the set of all nodes that can successfully send packets to node $j \in T_{v}$ with purported address $i \in T_{v}$ under a given filter configuration by $N_{v}^{i j}$. We also define two sets, $A_{v}^{1}$ and $A_{v}^{2}$, related to a possible attack on $v$ from a node within $T_{v}$. 
The set $A_{v}^{1}$ is defined by $A_{v}^{1}=N_{v}^{\ell v} \backslash O_{v}$, where $\ell \in \operatorname{argmax}_{i \in L_{v}}\left\{\left|N_{v}^{i v}\right|\right\}$. This is a maximal set of nodes not in the open set $O_{v}$, that can send packets to the root node of the subtree $T_{v}$ with a purported address of node $\ell \in L_{v}$. We can envision $A_{v}^{1}$ as the longest weighted path from a leaf node to the root node, excluding the weight of the root node, in the aggregated graph. Define $\bar{u} \in S_{v}$ as the child of $v$ for which $\ell \in L_{\bar{u}}$. Then the set $A_{v}^{2}$ is defined by $A_{v}^{2}=N_{v}^{q v} \backslash O_{v}$, where $q \in \operatorname{argmax}_{i \in L_{v} \backslash L_{\bar{u}}}\left\{\left|N_{v}^{i v}\right|\right\}$. $A_{v}^{2}$ is interpreted as the set of nodes in the second-longest weighted path in the aggregated graph from a leaf node to the root that does not intersect with nodes in $S_{\bar{u}}$, excluding the root weight. In our example in Figure 4.1, if nodes 3 and 4 are filtered, then $O_{1}=\{1,2\}, A_{1}^{1}=\{5,8,9,10\}$ and $A_{1}^{2}$ is $\{7\}$. (We cannot consider $\{6,11\}$ for $A_{1}^{2}$, since $\bar{u}=3$.) We refer to the cardinality of the set $A_{v}^{1}$ as the attack number of the subtree $T_{v}$ for a given filter configuration.

Since our dynamic programming algorithm works from the leaf nodes to the root node, our strategy is to retain promising partial solutions to subtrees that do not violate traceback feasibility conditions. Recall that the maximum traceback number in a subtree is given by the longest weighted path in the aggregated graph minus two. This longest path may or may not use the root node cluster. If it does not, we will use the principle of induction to claim feasibility of the solution since we will enforce feasibility of each subtree solution in the dynamic programming process. Else, the length of the longest path in the tree $T_{v}$ is given by $\left|A_{v}^{1}\right|+\left|A_{v}^{2}\right|+\left|O_{v}\right|$. We will accordingly enforce that $\left|A_{v}^{1}\right|+\left|A_{v}^{2}\right|+\left|O_{v}\right| \leq b+2$ for each $v$ encountered in our algorithm. The only vital characteristics needed for a candidate solution to $T_{v}$ after maintaining feasibility are the number of filters in the solution, $\left|A_{v}^{1}\right|$, and $\left|O_{v}\right|$.

The dynamic programming algorithm exploits the observation stated in Proposition 1 and the closed-form solutions of the chain and star networks to solve base case subtrees. A base case subtree is a subtree $T_{v}$ for some $v \in N$, that satisfies one of the following two properties:

1. $T_{v}$ is a chain with $\left|T_{v}\right| \geq 2$ and either $T_{p^{(v)}}$ is the root node $r$ or $T_{p^{(v)}}$ is not a chain, i.e. $\left|S_{p^{(v)}}\right| \geq 2$, or 
2. $S_{v}=L_{v}$, and $\left|L_{v}\right| \geq 2$.

In Figure 4.1, the base case subtrees are $T_{2}, T_{5}$, and $T_{6}$.

We store the salient features of candidate solutions to a subtree $T_{v}$ by a $\left(r_{v}+1\right) \times$ $\left(c_{v}+1\right)$ matrix of records $B^{v}$, in which the rows $0, \ldots, r_{v}=\left|D_{v}\right|-\left|L_{v}\right|+1$ represent the number of filters deployed in the subtree and the columns $0, \ldots, c_{v}=\left|D_{v}\right|+1$ represent the size of the open set for a candidate solution. An entry $b_{i j}^{v}$ of the matrix $B^{v}$ represents the minimum attack number for the subtree when $i$ filters are deployed so that the size of the open set is $j$. If deploying $\bar{\imath}$ filters in the subtree so that the size of the open set is $\bar{\jmath}$ violates the traceback limit requirement, we set $b_{\bar{\imath} \bar{\jmath}}^{v}$ to infinity. We also store the filter solution itself leading to each finite $b_{i j}^{v}$ entry.

To compute entries for the matrix $B^{v}$ corresponding to a base case subtree $T_{v}$, we start by initializing all entries to infinity. We then update only nondominated entries that do not violate the traceback limit. An entry $b_{i j}^{v}$ of the base case matrix $B^{v}$ dominates another element $b_{i \bar{\jmath}}^{v}$ if $b_{i j}^{v}+j \leq b_{i \bar{\jmath}}^{v}+\bar{\jmath}$ and $\bar{\jmath}>j$. For either star or chain base cases, we set the entry $b_{0,\left|D_{v}\right|+1}^{v}=0$ if $\left|D_{v}\right|+1 \leq b+2$. If $T_{v}$ is a star, we set $b_{10}^{v}=1$ and record $v$ as the filtered node corresponding to this entry. Else, $T_{v}$ is a chain, and for each row $i=1, \ldots, r_{v}$ of the matrix $B^{v}$, we set the entry $b_{i 0}^{v}=\left|D_{v}\right|+1-i$ if $\left|D_{v}\right|+1-i \leq b+2$. We arbitrarily record nodes $v$ and its descendants at levels $\ell_{v}+k$, for $k=1, \ldots, i$, as the solution corresponding to $b_{i 0}^{v}$.

Once we solve base case subtrees, we proceed to solve all those subtrees $T_{v}$ that satisfy the condition that for every $u \in S_{v}$ either $u \in L_{v}$, or $T_{u}$ has already been solved. In our example in Figure 4.1, $T_{3}$ satisfies this condition since both $T_{5}$ and $T_{6}$ are base case subtrees. We construct the matrix $B^{v}$ in two stages. We construct columns 1 through $c_{v}$ by allowing for positive open set sizes in the first stage, and in the second stage we construct column 0 using a procedure that assumes the placement of a filter at node $v$. 


\section{Computing matrix entries with a positive open set size.}

We employ a record-keeping scheme as an intermediate step to the construction of columns $1, \ldots, c_{v}$. Each record has five fields and is of the form $a=\left(a_{1}, a_{2}, a_{3}, a_{4}, a_{5}\right)$, where $a_{1}=\left|A_{v}^{1}\right|$ is the attack number, $a_{2}=\left|A_{v}^{2}\right|, a_{3}=\left|O_{v}\right|$ is the size of the open set, $a_{4}$ is the number of filters deployed, and $a_{5}$ indicates the number of child subtree solutions used to obtain the current record. Since we update records by considering the addition of one child's subtree to the solution at a time, the field $a_{5}$ denotes the stage of this recursion subroutine. The sum of the attack number and the open set size gives the maximum number of nodes from a subtree $T_{v}$ that could send packets with the same purported origin address to a node outside of the subtree. The sum of the first three fields minus two gives the maximum traceback number for the subtree $T_{v}$ under the filter configuration represented by the record $a$.

For notational ease we label nodes in $S_{v}$ as $u_{1}, u_{2}, \ldots, u_{s_{v}}$. We create an initial stage 1 record corresponding to each finite element of the solution matrix $B^{u_{1}}$. Let $b_{i j}^{u_{1}}$ be a finite element in the $i^{\text {th }}$ row and $j^{\text {th }}$ column of the matrix $B^{u_{1}}$. Then the record corresponding to this element is obtained as follows: $a_{1}=b_{i j}^{u_{1}}, a_{2}=0$, $a_{3}=j+1, a_{4}=i, a_{5}=1$. Given a traceback limit $b$, we only keep a record $a$ if $a_{1}+a_{2}+a_{3} \leq b+2$, due to traceback limit requirements. Otherwise, any solution derived from such a record will clearly violate the traceback limitation of $b$. After generating stage 1 records, we compute stage 2 records by combining stage 1 records and the solution from the subtree $T_{u_{2}}$.

In general, for each record from stage $k-1$, we create $n^{u_{k}}$ new records, where $n^{u_{k}}$ is the number of finite elements in the matrix $B^{u_{k}}$. Let a record from stage $k$ be denoted by $a^{k}=\left(a_{1}^{k}, a_{2}^{k}, a_{3}^{k}, a_{4}^{k}, a_{5}^{k}\right)$. For each record previously created from stage $k-1$, and for each finite element $b_{i j}^{u_{k}}$ of the matrix $B^{u_{k}}$, we compute a stage $k$ record as follows: $a_{1}^{k}=\max \left\{a_{1}^{k-1}, b_{i j}^{k}\right\}, a_{2}^{k}$ is the second-largest of $a_{1}^{k-1}, a_{2}^{k-1}$, and $b_{i j}^{k}, a_{3}^{k}=a_{3}^{k-1}+j, a_{4}^{k}=a_{4}^{k-1}+i$, and $a_{5}^{k}=k$.

At each stage $k$, we only keep feasible records that are not dominated by (or identical to) other records. A record $\bar{a}$ is said to be dominated by another record 
$\tilde{a}$ if we have $\bar{a}_{1} \geq \tilde{a}_{1}, \bar{a}_{2} \geq \tilde{a}_{2}, \bar{a}_{3} \geq \tilde{a}_{3}, \bar{a}_{4} \geq \tilde{a}_{4}$ and $\bar{a}_{5}=\tilde{a}_{5}$ with at least one strict inequality holding, or $\bar{a}_{1}>\tilde{a}_{1}$ and $\bar{a}_{2}<\tilde{a}_{2}, \bar{a}_{3} \geq \tilde{a}_{3}, \bar{a}_{4} \geq \tilde{a}_{4}, \bar{a}_{5}=\tilde{a}_{5}$. The dominance criteria in the second case follows from the following analysis. If $\bar{a}_{5}=\tilde{a}_{5}=s_{v}$ then $\tilde{a}$ is clearly preferable to $\bar{a}$. Else, by induction, suppose this claim is true at stages $k+1, \ldots, s_{v}$ and consider two records $\bar{a}^{k}$ and $\tilde{a}^{k}$ for which $\bar{a}_{1}^{k}>\tilde{a}_{1}^{k}$, $\bar{a}_{2}^{k}<\tilde{a}_{2}^{k}, \bar{a}_{3}^{k} \geq \tilde{a}_{3}^{k}, \bar{a}_{4}^{k} \geq \tilde{a}_{4}^{k}$, and $\bar{a}_{5}^{k}=\tilde{a}_{5}^{k}=k$. These records, when combined with a finite element $b_{i j}^{u_{k+1}}$ of the matrix $B^{u_{k+1}}$, produce two new records $\bar{a}^{k+1}$ and $\tilde{a}^{k+1}$ such that $\bar{a}_{3}^{k+1} \geq \tilde{a}_{3}^{k+1}, \bar{a}_{4}^{k+1} \geq \tilde{a}_{4}^{k+1}$, and $\bar{a}_{5}^{k+1}=\tilde{a}_{5}^{k+1}$. Additionally, one of the following three conditions must hold true: 1) $\bar{a}_{1}^{k+1}>\tilde{a}_{1}^{k+1}$ and $\left.\bar{a}_{2}^{k+1}=\tilde{a}_{2}^{k+1}, 2\right) \bar{a}_{1}^{k+1}=\tilde{a}_{1}^{k+1}$ and $\bar{a}_{2}^{k+1}>\tilde{a}_{2}^{k+1}$, or 3$) \bar{a}_{1}^{k+1}>\tilde{a}_{1}^{k+1}$ and $\bar{a}_{2}^{k+1}<\tilde{a}_{2}^{k+1}$. If condition 1 or condition 2 is true, then $\tilde{a}^{k+1}$ dominates $\bar{a}^{k+1}$ according to the first criterion. On the other hand, if condition 3 is true, then $\tilde{a}^{k+1}$ dominates $\bar{a}^{k+1}$ by induction. In any case, it is not necessary to retain record $\bar{a}^{k}$.

Once the records for stage $s_{v}$ have been computed, we populate entries of the matrix $B^{v}$ in columns $1, \ldots, c_{v}$ corresponding to nondominated records of stage $s_{v}$. Additionally, we record the actual filter placements leading to these values, which we can easily obtain by tracing back through our procedure. Initially, all entries of the matrix $B^{v}$ are initialized to infinity. From each nondominated record $a^{s_{v}}$, we obtain the matrix entry $b_{a_{4}^{s_{v}} a_{3}^{s_{v}}}^{v}=a_{1}^{s_{v}}$. We state a key observation regarding the number of nondominated records at the end of each stage $k, k=1, \ldots, s_{v}$, in the following proposition.

Proposition 2. The maximum number of nondominated records at the end of stage $k$ of the foregoing procedure, for $k=1, \ldots, s_{v}$, for any $v \in N$, is at most $n^{2}$.

Proof: Consider some $v \in N$ and computed stage $k$ records, and examine the set $H_{i j}$ of all records that have $a_{4}^{k}=i$ and $a_{3}^{k}=j, i \in\left\{0, \ldots, r_{v}\right\}, j \in\left\{1, \ldots, c_{v}\right\}$. If $\left|H_{i j}\right| \geq 2$, consider two records $\bar{a}^{k} \in H_{i j}$ and $\tilde{a}^{k} \in H_{i j}$. Without loss of generality, assume that $\bar{a}_{1}^{k} \geq \tilde{a}_{1}^{k}$. If $\bar{a}_{2}^{k} \geq \tilde{a}_{2}^{k}$, then the record $\bar{a}^{k}$ is dominated and can be discarded. If on the other hand, $\bar{a}_{2}^{k}<\tilde{a}_{2}^{k}$, then we consider the following two cases: 1) $\bar{a}_{1}^{k}=\tilde{a}_{1}^{k}$, and 2) $\bar{a}_{1}^{k}>\tilde{a}_{1}^{k}$. Case 1 implies that the record $\tilde{a}^{k}$ is dominated and 
may be discarded while case 2 implies that $\bar{a}^{k}$ is dominated. Continuing with this process iteratively, we retain only a single record in the set $H_{i j}$. Thus, we will have $\left|H_{i j}\right| \leq 1, \forall i \in\left\{0, \ldots, r_{v}\right\}$ and $\forall j \in\left\{1, \ldots, c_{v}\right\}$ and therefore, at most $n^{2}$ nondominated records at the end of stage $k$.

\section{Computing matrix entries with an open set size of zero.}

Next, we describe a procedure for calculating entries for column 0 of $B^{v}$, for some $v \in N$. Let $r_{u_{j}}$ be the minimum number of filters needed to attain perfect security in subtree $T_{u_{j}}$ for each $u_{j} \in S_{v}$, and define $\mu_{v}=\max _{j \in\left\{1, \ldots, s_{v}\right\}}\left\{r_{u_{j}}\right\}$, where again $s_{v}=\left|S_{v}\right|$. We start by constructing a $\mu_{v} \times s_{v}$ matrix $M$ from the matrices $B^{u_{j}}$, where the initial entries to $M$ are all infinity. The matrix $M$ is constructed by iterating through each finite value $b_{i k}^{u_{j}}$ found in $B^{u_{j}}$ and setting $m_{i j}=\min \left\{m_{i j}, b_{i k}^{u_{j}}+k\right\}$ for $i=0, \ldots, r_{u_{j}}$ and $j=1, \ldots, s_{u_{j}}$. If $r_{u_{j}}<\mu_{v}$ for some $j \in\left\{1, \ldots, s_{v}\right\}$, we set $m_{i j}=1$ for $i=r_{u_{j}}+1, \ldots, \mu_{v}$. The resulting matrix entries $m_{i j}$ represent the minimum attack number attainable in $T_{v}$ if $i$ filters are deployed.

We say an element $m_{i j}$ of the matrix $M$ is covered if we have at least $i+1$ filters deployed in the subtree $T_{u_{j}}$; otherwise, the element is uncovered. We denote the largest uncovered element in $M$ by $\hat{m}_{1}$ and the corresponding column index by $\hat{\jmath}_{1}$. Similarly, we denote the second-largest uncovered element in $M$ by $\hat{m}_{2}$ and its column index by $\hat{\jmath}_{2}$.

Solving for an element $b_{i 0}^{v}$ of the solution matrix $B^{v}$, for some $v \in N$, is equivalent to finding a distribution of $i-1$ filters amongst the columns of the matrix $M$ so that $\hat{m}_{1}$ is minimized, and the sum of $\hat{m}_{1}$ and $\hat{m}_{2}$ is at most $b+2$. We denote such a filter distribution by a vector $f^{i}=\left(f_{1}^{i}, \ldots, f_{s_{v}}^{i}\right)$, where each component $f_{j}^{i}$ represents the number of filters deployed in the subtree $T_{u_{j}}, u_{j} \in S_{v}$, from a total of $i$ filters available for deployment in $T_{v}$. If a feasible $f^{i}$ exists, we set $b_{i 0}^{v}=\hat{m}_{1}$; otherwise, we set $b_{i 0}^{v}=\infty$. An example of the matrix $M$ is shown in Table 4.1. In this example, $r_{u_{1}}=4, r_{u_{2}}=4, r_{u_{3}}=5, r_{u_{4}}=6, r_{u_{5}}=4$, and $\mu_{v}=6$.

We start the process with $i=r_{v}=1+\sum_{j=1}^{s_{v}} r_{u_{j}}$, implying that all non-leaf nodes are filtered. Hence, we set $\hat{m}_{1}=\hat{m}_{2}=1$ and (arbitrarily) set $\hat{\jmath}_{1}=1$ and $\hat{\jmath}_{2}=2$. 
Table 4.1: Example of a matrix $M$.

\begin{tabular}{c|ccccc} 
& 1 & 2 & 3 & 4 & 5 \\
\hline 0 & 4 & 8 & 10 & 28 & 8 \\
1 & 3 & 7 & 6 & 10 & 4 \\
2 & 2 & 4 & 4 & 9 & 3 \\
3 & 2 & 3 & 4 & 8 & 2 \\
4 & 1 & 1 & 2 & 4 & 1 \\
5 & 1 & 1 & 1 & 3 & 1 \\
6 & 1 & 1 & 1 & 1 & 1
\end{tabular}

We initialize $b_{i 0}^{v}=\hat{m}_{1}$ and the corresponding solution vector $f^{i}=\left(f_{1}^{i}, \ldots, f_{s_{v}}^{i}\right)=$ $\left(r_{u_{1}}, \ldots, r_{u_{s_{v}}}\right)$. We then proceed to calculate the entry $b_{i-1,0}^{v}$ from $f^{i}$ as follows. Let $\bar{\jmath} \in \operatorname{argmin}_{j \in J^{i}}\left\{m_{f_{j}^{i}-1, j}\right\}$, where $J^{i}$ is the set of all $j \in\left\{1, \ldots, s_{v}\right\}$ such that $f_{j}^{i}>0$. If $\hat{\jmath}_{1} \neq \bar{\jmath}$, set $\hat{\jmath}_{2}=\hat{\jmath}_{1}, \hat{m}_{2}=\hat{m}_{1}$, and $\hat{\jmath}_{1}=\bar{\jmath}$. Set $\hat{m}_{1}=m_{f_{\hat{\jmath}_{1}}-1, \hat{\jmath}_{1}}$ and $i=i-1$. If $\hat{m}_{1}+\hat{m}_{2} \leq b+2$, then set $b_{i 0}^{v}=\hat{m}_{1}$, and set the corresponding solution vector $f^{i}$ identical to $f^{i+1}$ except that $f_{\hat{\jmath}_{1}}^{i+1}$ is replaced with $f_{\hat{\jmath}_{1}}^{i+1}-1$. Record the corresponding filter placement as well. Repeat the process until we get $\hat{m}_{1}+\hat{m}_{2}>b+2$ or $i=1$. When $i=1$, all entries in column 0 of the solution matrix $B^{v}$ have been calculated and the process terminates. On the other hand, if $\hat{m}_{1}+\hat{m}_{2}>b+2$, at some index $i=i^{*}$, we attempt to find a feasible filter placement that minimizes $\hat{m}_{1}$ by rearranging the filters. (In our example in Table 4.1, if $b=10$, then $i^{*}=8$ with a solution vector $f^{8}=(0,1,1,4,1)$ and corresponding values $\hat{\jmath}_{1}=2, \hat{\jmath}_{2}=3$, $\hat{m}_{1}=7$, and $\hat{m}_{2}=6$.) If we cannot get a feasible filter placement after the filter rearrangement, we set $b_{i 0}^{v}=\infty$, for $i=0, \ldots, i^{*}$. If we do find a filter placement such that $\hat{m}_{1}+\hat{m}_{2} \leq b+2$, we store the solution and continue to decrement $i$.

The process of rearranging filters to obtain a feasible solution is accomplished by sequentially designating a column $j_{s}$ of $M, j_{s}=1, \ldots, s_{v}$, that is responsible for distributing filters to other columns. We make a temporary copy $\tilde{f}$ of the solution vector $f^{i}$ and initialize an incumbent solution vector $\bar{f}$ as a blank vector with an objective value of $\bar{m}=\infty$. We move a filter from column $j_{s}$ in solution $\tilde{f}$ to a column $j_{t} \in \operatorname{argmax}_{j \neq j_{s}}\left\{m_{\tilde{f}_{j}, j}\right\}$ by updating the values $\tilde{f}_{j_{s}}=\tilde{f}_{j_{s}}-1, \tilde{f}_{j_{t}}=\tilde{f}_{j_{t}}+1$, setting 
$\hat{m}_{1}=m_{\tilde{f}_{j_{s}}, j_{s}}$, and recomputing the value of $\hat{m}_{2}$. If $\hat{m}_{1}+\hat{m}_{2} \leq b+2$, we compare $\hat{m}_{1}$ to $\bar{m}$ and set $\bar{f}=\tilde{f}$ if $\hat{m}_{1}<\bar{m}$. We update $j_{s}=j_{s}+1$, reset $\tilde{f}=f^{i}$ and continue searching for a better solution, until $j_{s}=s_{v}$. On the other hand, if $\hat{m}_{1}+\hat{m}_{2}>b+2$, we continue taking filters from column $j_{s}$, one at a time, each time recomputing $j_{t}$ and updating the values of $\tilde{f}_{j_{s}}, \tilde{f}_{j_{t}}, \hat{m}_{1}$, and $\hat{m}_{2}$ until one of the following three conditions is true: 1) we have a feasible solution, 2) $\tilde{f}_{j_{s}}=0$, or 3) $\hat{m}_{2}=1$. In the latter two cases, we update $j_{s}=j_{s}+1$, reset $\tilde{f}=f^{i}$ and restart the process. When this step has been completed for $j_{s}=s_{v}$ and the procedure terminates, we return $\bar{f}$ as our solution if $\bar{m}<\infty$.

We illustrate the filter rearranging process by calculating the entry $b_{8,0}$ from the matrix $M$ in Table 4.1 using $f^{8}=(0,1,1,4,1)$ and $b=10$. At this stage $\hat{m}_{1}=7$ and $\hat{m}_{2}=6$, which violates the traceback requirement. We start by setting $\tilde{f}=(0,1,1,4,1), \bar{m}=\infty$, and $j_{s}=1$. Since $\tilde{f}_{1}=0$, we set $j_{s}=2$, and move a filter from column 2 to column $j_{t}=3$. After updating the values we have $\tilde{f}=(0,0,2,4,1)$, $\hat{m}_{1}=8$, and $\hat{m}_{2}=4$, which is feasible. Since $\hat{m}_{1}<\bar{m}$, we update the incumbent solution $\bar{f}=\tilde{f}$ and the objective $\bar{m}=8$. We reset $\tilde{f}=f^{8}$ and update $j_{s}=3$. We move a filter from column $j_{s}=3$ to column $j_{t}=2$ which yields the values $\tilde{f}=(0,2,0,4,1), \hat{m}_{1}=10$, and $\hat{m}_{2}=6$. This solution is infeasible, and since $\tilde{f}_{3}=$ 0 , we reset $\tilde{f}=f^{8}$ and update $j_{s}=4$. We now move filters from column $j_{s}=4$ to column $j_{t}=2$, then 3 , then 5 , and then 3 , without attaining feasibility. At this point $\tilde{f}_{4}=0$, we reset $\tilde{f}$ to $f^{8}$ and increment $j_{s}$ to 5 . We then move a filter from column 5 to column 2, but again we do not attain a feasible solution. The procedure terminates with solution $\bar{f}$ and objective $\bar{m}=8$.

The column 0 computation procedure is summarized in the following algorithm:

Step 0: Initialization. Set $i=1+\sum_{j=1}^{s_{v}} r_{u_{j}}, \hat{m}_{1}=\hat{m}_{2}=1, \hat{\jmath}_{1}=1, \hat{\jmath}_{2}=2$, $f^{i}=\left(f_{1}^{i}, \ldots, f_{s_{v}}^{i}\right)=\left(r_{u_{1}}, \ldots, r_{u_{s_{v}}}\right)$. Set $b_{i 0}^{v}=\hat{m}_{1}$ and go to step 1 .

Step 1: Let $\bar{\jmath} \in \operatorname{argmin}_{j \in J^{i}}\left\{m_{f_{j}^{i}-1, j}\right\}$, where $J^{i}=\{$ all $j \in$ $\left\{1, \ldots, s_{v}\right\}$ such that $\left.f_{j}^{i}>0\right\}$. If $\hat{\jmath}_{1} \neq \bar{\jmath}$, set $\hat{\jmath}_{2}=\hat{\jmath}_{1}, \hat{m}_{2}=\hat{m}_{1}$, and $\hat{\jmath}_{1}=\bar{\jmath}$. Set $\hat{m}_{1}=m_{{\hat{\jmath_{1}}}_{1}-1, \hat{\jmath}_{1}}, f^{i-1}=f^{i}$ with the component $f_{\hat{\jmath}_{1}}^{i-1}$ replaced by 
$f_{\hat{\jmath}_{1}}^{i}-1$, and $i=i-1$. If $\hat{m}_{1}+\hat{m}_{2}>b+2$ go to step 2. Otherwise, set $b_{i 0}^{v}=\hat{m}_{1}$. If $i=1$, terminate the process; otherwise, repeat step 1 .

Step 2: Let $\tilde{f}=f^{i}, j_{s}=1, \bar{f}=\emptyset$, and $\bar{m}=\infty$. Go to step 3 .

Step 3: If $\tilde{f}_{j_{s}}=0$ or $\hat{m}_{2}=1$, go to step 4 , otherwise let $j_{t} \in \operatorname{argmax}_{j \neq j_{s}}\left\{m_{\tilde{f}_{j}, j}\right\}$. Update the values $\tilde{f}_{j_{s}}=\tilde{f}_{j_{s}}-1, \tilde{f}_{j_{t}}=\tilde{f}_{j_{t}}+1$. Recompute the values of $\hat{j}_{2}$ and $\hat{m}_{2}$. If $\hat{m}_{1}+\hat{m}_{2}>b+2$ repeat step 3 . Otherwise we have a feasible solution with attack number $\hat{m}_{1}$. If $\hat{m}_{1}<\bar{m}$, set $\bar{f}=\tilde{f}$ and $\bar{m}=\hat{m}_{1}$. Go to step 4 .

Step 4: If $j_{s}<s_{v}$ set $j_{s}=j_{s}+1, \tilde{f}=f^{i}$, and go to step 3. Else, if $\bar{m}=\infty$ then set $b_{k 0}^{v}=\infty$ for $k=0, \ldots, i$ and terminate; otherwise, set $b_{i 0}^{v}=\bar{m}$ and $f^{i-1}=f^{i}$ with component $f_{k}^{i-1}=f_{k}^{i}-1$ for $k \in \operatorname{argmin}_{j \in J^{i}}\left\{m_{f_{j}^{i}-1, j}\right\}$. Set $i=i-1$. If $i=1$, terminate the procedure; otherwise, go to step 2 .

We now discuss why the column 0 computation procedure yields the optimal set of minimum attack numbers $b_{i 0}^{v}$ for $i=1, \ldots, 1+\sum_{j=1}^{s_{v}} r_{u_{j}}$, for any $v \in N$. Note that the initial solution vector $f^{i}$, for some $i \in\left\{1, \ldots, 1+\sum_{j=1}^{s_{v}} r_{u_{j}}\right\}$ is obtained by greedily minimizing the maximum attack number in any column with no regard to the feasibility condition that $\hat{m}_{1}+\hat{m}_{2} \leq b+2$. Hence, if $f^{i}$ is feasible, it must represent an optimal allocation of filters across the subtrees $T_{u_{j}}$ for $j=1, \ldots, s_{v}$.

Otherwise, note that while $f^{i}$ minimizes the value for $\hat{m}_{1}$, it does not necessarily minimize $\hat{m}_{1}+\hat{m}_{2}$. However, for any solution vector $\tilde{f}$ such that $\tilde{f}_{j_{1}}<f_{j_{1}}^{i}$ and $\tilde{f}_{j_{2}}<$ $f_{j_{2}}^{i}$ for $1 \leq j_{1}<j_{2} \leq s_{v}$, we have that $\tilde{f}$ must be infeasible. This claim follows from the fact that by construction of $f^{i}$, we have that $m_{f_{j}^{i}-1, j} \geq \hat{m}_{1}$ for each $j=1, \ldots, s_{v}$, and hence $m_{f_{j_{1}}^{i}-1, j_{1}}+m_{f_{j_{2}}^{i}-1, j_{2}}>b+2$. Therefore, if $\hat{m}_{1}+\hat{m}_{2}>b+2$ in the solution $f^{i}$, any feasible solution $\bar{f}$ must have that $\bar{f}_{j_{1}}<f_{j_{1}}^{i}$ for some $j_{1} \in\left\{1, \ldots, s_{v}\right\}$, and $\bar{f}_{j} \geq f_{j}^{i}$ for all other $j \in\left\{1, \ldots, s_{v}\right\}, j \neq j_{1}$. The column 0 computation procedure in steps $2-4$ selects one column $j_{1}$ at a time to be $\hat{\jmath}_{1}$, and then minimizes $\hat{m}_{2}$ in a greedy fashion. Hence, if an optimal solution exists, it is found during the examination of one such $j_{1} \in\left\{1, \ldots, s_{v}\right\}$. 


\subsubsection{Complexity of the dynamic program}

We first derive the complexity of the procedure that computes columns $1, \ldots, c_{v}$ of the matrix $B^{v}$. For any $v \in N, B^{v}$ could have at most $n^{2}$ finite elements. In addition, Proposition 2 limits the number of nondominated records at the end of stage $k, k=1, \ldots, s_{v}$, to at most $n^{2}$. Therefore, we can calculate stage $k+1$ records, $k=1, \ldots, s_{v}-1$, in $O\left(n^{4}\right)$ computations. Since the number of stages in our recordkeeping scheme is determined by $s_{v}$, only $O(n)$ of these stages are required to solve the entire tree. This results in a computational complexity of $O\left(n^{5}\right)$ for computing columns $1, \ldots, s_{v}$ of all matrices $B^{v}, v \in N$.

Next, we compute the complexity of the procedure that populates column 0 of a matrix $B^{v}, v \in N$. When solving for an entry of column 0 , if we determine the solution within step 1 , then we expend only $O(n)$ computations to find this value, due to a single search over $O(n)$ columns of the matrix $M$. On the other hand, determining the solution in steps $2-4$ involves rearranging the deployed filters. The process of moving a filter from column $j_{s}$ to column $j_{t}$ is accomplished in $O(n)$ computations. Since there are $O(n)$ filters in columns $1, \ldots, s_{v}$, computing a single entry of $B^{v}, v \in N$, requires $O\left(n^{2}\right)$ computations. Therefore, solving for all column 0 elements of a solution matrix $B^{v}$ is done in $O\left(n^{3}\right)$ computations. The computational complexity of solving for column 0 of all solution matrices in the tree graph is thus only $O\left(n^{4}\right)$, and so the overall complexity of the dynamic programming algorithm is $O\left(n^{5}\right)$.

\subsection{Computational Results}

In this section, we present computational results for both the dynamic programming procedure and the mixed-integer programming model. Seven sets of randomly generated problem instances were used to evaluate the performance of the dynamic programming procedure. For each instance set, 10 instances were generated, for a total of 70 instances. Each instance was obtained by computing a minimum spanning tree of a randomly generated graph with random edge weights. We refer to 
an instance set containing instances with $n$ nodes as "Inst_ $n$." Thus, we denote the seven instance sets we generated by Inst_15, Inst_30, Inst_45, Inst_100, Inst_150, Inst_200, and Inst_250. We assume complete communication in all instances as required by the dynamic programming algorithm for trees.

Instance sets Inst_15, Inst_30, and Inst_45 were solved by both mixed-integer programming and the dynamic programming algorithm, given several different traceback limit values $b$. Tables $4.2-4.4$ show average computational times and standard deviations for the three instance sets, given in units of CPU seconds. The column "MIP CPU" gives the average CPU times to solve problem instance, while "MIP Std Dev" gives the standard deviation of the CPU times over the 10 instances. The columns "DP CPU" and "DP Std Dev" are defined similarly for the dynamic programming algorithm. Entries in the tables with a value of zero correspond to those values that were less than 0.005 seconds. From these tables, we observe that the computational times for the dynamic programming algorithm change very little as the instance sizes increase. By contrast, there is clearly a sharp increase in computational time required to solve the same instances by mixed-integer programming as the problem size increase from 15 nodes to 30 nodes, and from 30 nodes to 45 nodes.

Another observation from Tables 4.2-4.4 regards the peak of computation times for the two procedures. For the mixed-integer programming model, this peak occurs when $b$ is roughly about half of $n$. This is because few filters are required when $b$ becomes moderately large, and many small subsets of $N$ serve as alternate optimal solutions. Towards the extreme values of $b$, the number of such subsets diminishes, more variables can be fixed to 0 or 1 using standard preprocessing algorithms, and hence lower computational times are obtained as a result. The dynamic programming algorithm on the other hand shows increasing computational times as $b$ increases. This trend is due to the fact that as the traceback limit increases, the number of feasible records also increases. As a result, the amount of computational effort required to compute subsequent records also increases, approaching the worst-case analysis as described in Section 4.2.3. 
The set of computational runs executed for Table 4.5 demonstrates the efficacy of our dynamic programming algorithm on larger instances. Clearly, our dynamic programming algorithm is capable of solving much larger instances than the mixedinteger programming approach given the same amount of computational time. Also, the set of problems for which our dynamic programming algorithm runs most efficiently are once again those for which $b$ is relatively small, as we would expect to encounter in practical settings.

Table 4.2: Average CPU times and standard deviations for Inst_15, in seconds.

\begin{tabular}{|c|c|c|c|c|}
\hline$b$ & MIP CPU & MIP Std Dev & DP CPU & DP Std Dev \\
\hline 0 & 0.18 & 0.01 & 0.01 & 0.01 \\
1 & 0.41 & 0.08 & 0.01 & 0.00 \\
2 & 0.29 & 0.02 & 0.01 & 0.00 \\
3 & 0.30 & 0.03 & 0.01 & 0.00 \\
4 & 0.37 & 0.09 & 0.01 & 0.00 \\
5 & 0.46 & 0.11 & 0.01 & 0.01 \\
6 & 0.53 & 0.27 & 0.01 & 0.01 \\
7 & 0.51 & 0.11 & 0.01 & 0.01 \\
8 & 0.55 & 0.15 & 0.01 & 0.00 \\
9 & 0.52 & 0.17 & 0.01 & 0.00 \\
10 & 0.56 & 0.24 & 0.01 & 0.01 \\
11 & 0.33 & 0.02 & 0.01 & 0.00 \\
12 & 0.33 & 0.01 & 0.01 & 0.01 \\
13 & 0.20 & 0.01 & 0.00 & 0.01 \\
\hline
\end{tabular}


Table 4.3: Average CPU times and standard deviations for Inst_30, in seconds.

\begin{tabular}{|c|c|c|c|c|}
\hline$b$ & MIP CPU & MIP Std Dev & DP CPU & DP Std Dev \\
\hline 0 & 1.58 & 0.06 & 0.01 & 0.01 \\
2 & 3.82 & 0.78 & 0.01 & 0.01 \\
4 & 5.58 & 3.26 & 0.00 & 0.00 \\
6 & 10.95 & 5.39 & 0.01 & 0.00 \\
8 & 13.02 & 4.77 & 0.01 & 0.01 \\
10 & 21.96 & 8.99 & 0.01 & 0.01 \\
12 & 26.87 & 22.26 & 0.01 & 0.00 \\
14 & 23.10 & 11.10 & 0.01 & 0.01 \\
16 & 17.82 & 14.58 & 0.01 & 0.01 \\
18 & 23.75 & 15.55 & 0.01 & 0.01 \\
20 & 24.60 & 14.41 & 0.01 & 0.01 \\
22 & 19.69 & 9.79 & 0.01 & 0.01 \\
24 & 5.32 & 3.07 & 0.02 & 0.01 \\
26 & 4.37 & 0.06 & 0.02 & 0.01 \\
\hline
\end{tabular}

Table 4.4: Average CPU times and standard deviations for Inst_45, in seconds.

\begin{tabular}{|c|c|c|c|c|}
\hline$b$ & MIP CPU & MIP Std Dev & DP CPU & DP Std Dev \\
\hline 0 & 6.23 & 0.28 & 0.01 & 0.00 \\
3 & 89.49 & 57.81 & 0.01 & 0.00 \\
6 & 107.83 & 105.02 & 0.01 & 0.00 \\
9 & 205.91 & 90.29 & 0.01 & 0.00 \\
12 & 224.98 & 150.51 & 0.01 & 0.01 \\
15 & 219.74 & 169.18 & 0.02 & 0.01 \\
18 & 268.64 & 112.81 & 0.02 & 0.01 \\
21 & 257.80 & 212.23 & 0.02 & 0.02 \\
24 & 305.86 & 157.66 & 0.03 & 0.02 \\
27 & 346.70 & 207.91 & 0.04 & 0.03 \\
30 & 331.24 & 312.18 & 0.05 & 0.03 \\
33 & 200.67 & 141.39 & 0.05 & 0.04 \\
36 & 218.07 & 73.15 & 0.06 & 0.04 \\
39 & 27.71 & 0.92 & 0.05 & 0.05 \\
\hline
\end{tabular}


Table 4.5: Average CPU seconds required to solve instance sets with 100, 150, 200, and 250 nodes.

\begin{tabular}{|c|c||c|c||c|c||c|c|}
\hline \multicolumn{2}{|c||}{ Inst_100 } & \multicolumn{2}{c||}{ Inst_150 } & \multicolumn{2}{c||}{ Inst_200 } & \multicolumn{2}{c|}{ Inst_250 } \\
\hline$b$ & Avg CPU & $b$ & Avg CPU & $b$ & Avg CPU & $b$ & Avg CPU \\
\hline 0 & 0.01 & 0 & 0.01 & 0 & 0.01 & 0 & 0.02 \\
7 & 0.01 & 10 & 0.03 & 14 & 0.13 & 17 & 0.36 \\
14 & 0.05 & 20 & 0.22 & 28 & 1.36 & 34 & 4.75 \\
21 & 0.15 & 30 & 0.80 & 42 & 5.00 & 51 & 19.06 \\
28 & 0.35 & 40 & 1.89 & 56 & 11.02 & 68 & 46.28 \\
35 & 0.62 & 50 & 3.48 & 70 & 18.98 & 85 & 82.59 \\
42 & 0.94 & 60 & 5.41 & 84 & 27.80 & 102 & 125.23 \\
49 & 1.28 & 70 & 7.51 & 98 & 36.36 & 119 & 169.55 \\
56 & 1.60 & 80 & 9.56 & 112 & 44.22 & 136 & 209.79 \\
63 & 1.89 & 90 & 11.53 & 126 & 50.91 & 153 & 244.47 \\
70 & 2.14 & 100 & 13.14 & 140 & 56.42 & 170 & 275.86 \\
77 & 2.32 & 110 & 14.44 & 154 & 60.49 & 187 & 297.79 \\
84 & 2.42 & 120 & 15.51 & 168 & 63.46 & 204 & 312.72 \\
91 & 2.49 & 130 & 16.11 & 182 & 65.78 & 221 & 323.29 \\
98 & 2.54 & 140 & 16.59 & 196 & 67.18 & 238 & 331.51 \\
\hline
\end{tabular}

\subsection{Summary}

In this Chapter, we presented a polynomial-time dynamic programming algorithm for solving the GMFPP on tree networks. The algorithm works by examining subtrees starting from leaf nodes, and progressing systematically towards the root node. We showed that the computational complexity of the dynamic program is $O\left(n^{5}\right)$. Computational results showed that the algorithm can solve much larger problem instances within reasonable computational limits than can a mixed-integer programming technique. 


\section{CHAPTER 5}

\section{Valid Inequalities and Heuristic Algorithms for the GMFPP.}

In this chapter, we derive some valid inequalities for the GMFPP mixed-integer programming model by identifying polynomially solvable subgraphs of the underlying communication. We show that these inequalities are facet-inducing on the respective subgraphs. In addition, we derive valid inequalities based on the dynamic programming procedure presented in Chapter 4. Finally we provide three heuristics for solving the GMFPP. We evaluate the effectiveness of adding these inequalities to the mixed-integer programming model and assess the performance of the heuristics against optimal solutions from the mixed-integer programming model.

\subsection{Independent Subgraphs and Valid Inequalities}

In this section, we focus our attention on tightening (3.1) by the addition of valid inequalities. These inequalities are derived by examining polynomially-solvable independent subgraphs of the communication network. An independent subgraph $G^{\prime}=\left(N^{\prime}, A^{\prime}\right)$ is a subgraph of a communication network $G=(N, A)$ such that if nodes $u \in N^{\prime}$ and $v \in N^{\prime}$ communicate, i.e., $(u, v) \in C$, then all arcs in each $u-v$ path belong to $A^{\prime}$. All subgraphs in this section are assumed to be independent subgraphs, and the results we present are valid for both maximal and semi-maximal filters.

In Chapter 4, we identified some special network topologies that have a closedform solution to the GMFPP. These include the chain, star, and clique network topologies, and assume that all message pathways are bidirectional (hence graphs contain undirected edges instead of directed arcs). We make the same assumption for the subgraphs investigated in this section. The chain and star graphs are special cases of a tree network. A chain is a tree with only two leaf nodes while a star 
is a tree in which all but one node are leaf nodes. A clique is a graph in which there exists an edge between all distinct node pairs. Closed-form solutions for these special network topologies are briefly summarized here for completeness.

For a given traceback limit $b$ such that $b \leq n-3$, the minimum number of filters needed to attain the level of security dictated by $b$ in a chain network is $|F|=n-b-2$. These filters are placed on any subset of nodes that does not include leaf nodes. For a star topology, if $b \leq n-3$, we only need $|F|=1$, since placing a filter on the non-leaf node of the star network provides perfect security. In a clique network topology, to achieve a security level prescribed by the traceback limit $b \leq n-3$, we must deploy $|F|=n-b-1$ filters.

Define $Y$ as the GMFPP polytope projected onto the $y$-variables, i.e., $Y$ is the convex hull of all $y$-vectors such that there exists a feasible completion $w$ given $y$ in model (3.1). (We refer to $Y$ as the GMFPP $y$-polytope in the following discussion.) The following proposition establishes the dimensionality of $Y$.

Proposition 3. For a given traceback limit $b \geq 1$, the dimension of $Y$ equals $n$.

Proof. We prove this claim by identifying $n+1$ affinely independent vectors that belong to $Y$. First observe that filtering all nodes but one results in a traceback number of at most one, and hence, is feasible when $b \geq 1$. Let $y^{i}$ denote the solution in which all nodes except node $i$ are filtered, and let $y^{0}$ denote the solution in which all nodes are filtered. Now consider a set of vectors, $y^{1}-y^{0}, \ldots, y^{n}-y^{0}$. Observe that $y^{i}-y^{0}=-e_{i}$, where $e_{i}$ is an $n$-dimensional vector with all components equal to zero except for the $i^{\text {th }}$ component, which equals 1 . These vectors are clearly linearly independent, implying that our $n+1$ feasible points are affinely independent. This completes the proof.

Remark 1. When $b=0$, the dimension of the GMFPP polytope is not necessarily $n$. For example, a chain network has only four feasible points when $b=0$. These correspond to filtering all non-leaf nodes and 1) none of the leaf nodes, 2) one leaf node, 3) the other leaf node, and 4) both leaf nodes. Thus, for $n \geq 4$, the polytope $Y$ is not full dimensional. 
The process of deriving valid inequalities to tighten formulation (3.1) involves identifying star, chain, and clique independent subgraphs of the communication network. For a star subgraph on nodes $N_{s}$ centered at node $c$ such that $\left|N_{s}\right| \geq b+3$, the following star inequality is valid to formulation (3.1).

$$
\left(\left|N_{s}\right|-b\right) y_{c}+\sum_{i \in N_{s} \backslash\{c\}} y_{i} \geq\left|N_{s}\right|-b .
$$

The validity of the star inequality follows from the following observation. Suppose by contradiction that there exists a feasible solution $y^{*}$ in which $\left(\left|N_{s}\right|-b\right) y_{c}^{*}+$ $\sum_{i \in N_{s} \backslash\{c\}} y_{i}^{*}<\left|N_{s}\right|-b$. Let $L_{s}$ denote the set of filtered leaf nodes of the independent star in this solution. Clearly, $y_{c}^{*}=0$ since $y^{*}$ does not satisfy (5.1), and $\left|L_{s}\right| \geq 2$, since otherwise one leaf node could be attacked by all but two nodes, and $b \leq\left|N_{s}\right|-3$. Let $\ell_{1}$ and $\ell_{2}$ belong to $L_{s}$, and consider an attack on $\ell_{1}$ in which nodes forge their origin address as node $\ell_{2}$. All nodes in $N_{s} \backslash L_{s}$ can make such an attack without being filtered, contradicting the feasibility of $y^{*}$ as it violates the constraint set (3.1b).

Proposition 4. The star inequality (5.1) is facet-inducing to the GMFPP y-polytope of star subgraphs for traceback limit values of $2 \leq b \leq\left|N_{s}\right|-3$.

Before proving Proposition 4, we state and prove the following lemma as a precursor.

Lemma 3. For a given inequality $\sum_{j=1}^{p} y_{j} \geq \hat{b}, y_{j} \in\{0,1\} \forall j=1, \ldots, p$, and $1 \leq \hat{b} \leq p-1$, if setting any subset of $\hat{b} y$-variables to 1 is feasible to some polyhedral $\tilde{Y}$, then the inequality defines a $(p-1)$-dimensional face to $\tilde{Y}$.

Proof. We prove our claim by identifying $p$ linearly independent points satisfying $\sum_{j=1}^{p} y_{j}=\hat{b}$. Consider the following two sets of points: a) $y^{i}$ such that $y_{j}^{i}=1$ for $j \in\{1, \ldots, \hat{b}+1\} \backslash\{i\}$, and $y_{j}^{i}=0$ otherwise, for $i=1, \ldots, \hat{b}+1$, and b) $y^{i}$ such that $y_{j}^{i}=1$, for $j \in\{1, \ldots, \hat{b}-1\} \cup\{i\}$, and $y_{j}^{i}=0$ otherwise, for $i=\hat{b}+2, \ldots, p$. Now consider the system of linear equations $\sum_{i=1}^{p} y_{j}^{i} \alpha_{i}=0$ for $j=1, \ldots, p$, where $\alpha_{i} \in \mathbb{R}$ for $i=1, \ldots, p$. We obtain $\alpha_{i}=0$ for $i=\hat{b}+2, \ldots, p$ by simply inspecting equations $\hat{b}+2, \ldots, p$. From equations $1, \ldots, \hat{b}+1$, we obtain $\alpha_{1}=\alpha_{2}=\cdots=\alpha_{\hat{b}+1}$ 
by subtracting the equation $\sum_{i=1}^{p} y_{k}^{i} \alpha_{i}=0$ from $\sum_{i=1}^{p} y_{1}^{i} \alpha_{i}=0$, for $k=2, \ldots, \hat{b}+1$. Substituting these $\alpha$-values into the equation $\sum_{i=1}^{p} y_{k}^{i} \alpha_{i}=0$, where $k \in\{2, \ldots, \hat{b}+$ $1\}$, yields $\alpha_{i}=0$, for $i=1, \ldots, p$ as the unique solution to the system of equations. Hence, these $p$ points are affinely (in fact, linearly) independent, and form a $(p-1)$ dimensional face on $\tilde{Y}$. This completes the proof.

Proof of Proposition 4. Without loss of generality, assume that $N_{s}=\{1, \ldots, c=$ $\left.\left|N_{s}\right|\right\}$. We generate the first $c-1$ points according to Lemma 3 using $\hat{b}=c-b$ and we choose $y^{c}$ such that $y_{c}^{c}=1$ and $y_{j}^{c}=0$, for $j=1, \ldots, c-1$. Consider the system of equations $\sum_{i=1}^{c} y_{j}^{i} \alpha_{i}=0$, for $j=1, \ldots, c$. We obtain $\alpha_{c}=0$ from equation $c$ by inspection, and the result $\alpha_{1}=\alpha_{2}=\cdots=\alpha_{\left|N_{s}\right|-1}=0$ immediately follows from Lemma 3. This completes the proof.

Remark 2. Observe that for $b=0$ and $b=1$, the star inequality (5.1) is not facetinducing to the GMFPP $y$-polytope of a star subgraph. When $b=0$, the only binding feasible point to (5.1) is $y^{c}$, and when $b=1$, there are only two feasible binding points to (5.1): $y^{c}$ and the point $\bar{y}^{c}$, where $\bar{y}^{c}$ is a point with all components equal to one except $\bar{y}_{c}^{c}=0$, corresponding to filtering only leaf nodes. If $b=0, y_{c} \geq 1$ is a facet that dominates the star inequality, whereas if $b=1$, the star inequality can be disaggregated into the stronger representation $y_{i} \geq\left(1-y_{c}\right), \forall i \in N_{s} \backslash\{c\}$.

For a chain subgraph on node set $N_{h}$ with leaf nodes $\ell_{1}$ and $\ell_{2}$ such that $\left|N_{h}\right| \geq$ $b+3$, the following chain inequality is valid to the GMFPP:

$$
\sum_{i \in N_{h} \backslash\left\{\ell_{1}, \ell_{2}\right\}} y_{i} \geq\left|N_{h}\right|-b-2 .
$$

The validity of the chain inequality can be verified as follows. Suppose there exists a feasible solution to the GMFPP with $\sum_{i \in N_{h} \backslash\left\{\ell_{1}, \ell_{2}\right\}} y_{i}<\left|N_{h}\right|-b-2$. Denote the set of unfiltered non-leaf nodes on the chain subgraph by $\bar{N}_{h}$. Observe that $\sum_{i \in N_{h} \backslash\left\{\ell_{1}, \ell_{2}\right\}} y_{i}<\left|N_{h}\right|-b-2$ implies that $\left|\bar{N}_{h}\right| \geq b+1$. Now consider the possible attacks on node $\ell_{1}$ with purported origin address of $\ell_{2}$. All the nodes in $\bar{N}_{h}$ can attack $\ell_{1}$ with forged origin address of node $\ell_{2}$ regardless of the filter placement 
(and regardless of the presence of filters on leaf nodes) since deployed filters cannot distinguish legitimate packets from node $\ell_{2}$ from forged ones. However, this contradicts the feasibility of such a point with respect to constraint (3.1b).

Proposition 5. The chain inequality (5.2) is facet-inducing to the GMFPP $y$ polytope of chain subgraphs for $0 \leq b \leq\left|N_{h}\right|-3$.

Proof. Assume without loss of generality that $N_{h}=\left\{1, \ldots,\left|N_{h}\right|\right\}$, with nodes 1 and $\left|N_{h}\right|$ being leaf nodes. When $b=0$, the proof follows immediately since all points feasible to the chain subgraph are binding on the chain inequality. For $b \geq 1$, consider the points $y^{1}$ such that $y_{j}^{1}=1$ for $j=1, \ldots,\left|N_{h}\right|-b-1$ and $y_{j}^{1}=0$ otherwise; $y^{\left|N_{h}\right|}$ such that $y_{j}^{\left|N_{h}\right|}=1$ for $j \in\left\{2, \ldots,\left|N_{h}\right|-b-1\right\} \cup\left\{\left|N_{h}\right|\right\}$, and $y_{j}^{\left|N_{h}\right|}=0$ otherwise; and $y^{i}$, for $i=2, \ldots,\left|N_{h}\right|-1$, generated according to Lemma 3 with $\hat{b}=\left|N_{h}\right|-b-2$ on the index set $\left\{2, \ldots,\left|N_{h}\right|-1\right\}$. (Thus, we have $y_{j}^{i}=1$ for $j \in\{2, \ldots, \hat{b}+2\} \backslash\{i\}$, and $y_{j}^{i}=0$ otherwise, for $i=2, \ldots, \hat{b}+2$; and $y_{j}^{i}=1$ for $j \in\{2, \ldots, \hat{b}\} \cup\{i\}$, and $y_{j}^{i}=0$ otherwise, for $i=\hat{b}+3, \ldots,\left|N_{h}\right|-1$.) Now consider the system of equations $\sum_{i=1}^{\left|N_{h}\right|} y_{j}^{i} \alpha_{i}=0$ for $j=1, \ldots,\left|N_{h}\right|$. We obtain $\alpha_{1}=\alpha_{\left|N_{h}\right|}=0$ simply by inspecting equations 1 and $\left|N_{h}\right|$, respectively, and we obtain $\alpha_{2}=\cdots=\alpha_{\left|N_{h}\right|-1}=0$ using the results from Lemma 3. The dimension of the face induced by (5.2) equals to $\left|N_{h}\right|-1$, and thus (5.2) is facet-inducing to the GMFPP $y$-polytope on a chain. This completes the proof.

For a clique with node set $N_{q}$ such that $\left|N_{q}\right| \geq b+3$, the following clique inequality is valid to (3.1).

$$
\sum_{i \in N_{q}} y_{i} \geq\left|N_{q}\right|-b-1
$$

This inequality is valid, since if $\left|N_{q}\right|-b-2$ or fewer filters are deployed, then there exist at least $b+2$ unfiltered nodes. However, $b+1$ of the unfiltered nodes could attack the other unfiltered node using any origin address other than those in their set of $b+2$ nodes. (Another such node exists since $\left|N_{q}\right| \geq b+3$.) Hence (5.3) is a valid inequality. 
Proposition 6. The clique inequality (5.3) is facet-inducing to the GMFPP $y$ polytope of clique subgraphs for $b \leq\left|N_{q}\right|-3$.

Proof. Follows directly from Lemma 3 using $\hat{b}=\left|N_{q}\right|-b-1$

In addition to independent subgraphs, if the routing policy is such that for every node $d \in N$, the routing of nodes $u \in N \backslash\{d\}$ to $d, \forall(u, d) \in C$, form an in-tree rooted at node $d$, we can modify the dynamic programming procedure in Chapter 4 for solving tree networks to derive some valid inequalities and compute a lower bound on the optimal number of filters needed to solve the GMFPP. We examine the in-tree subgraph corresponding to each destination node $d \in N$ to determine a minimum cardinality set of nodes $F_{d}$ such that the traceback number of any attack on node $d$ is not more than $b$. A lower bound on the optimal number of filters required to solve the GMFPP is then computed as $L B=\max _{d \in N}\left\{\left|F_{d}\right|\right\}$.

This lower-bounding concept can be strengthened by the following procedure. For each node $u \in F_{d}, \forall d \in N$, we resolve the in-tree filtering problem with the assumption that node $u$ is left unfiltered. Letting $F_{d}^{u}$ denote the resulting new optimal solution, the following inequality is valid to (3.1):

$$
\sum_{i \in N \backslash\{u\}} y_{i}+\left(\left|F_{d}^{u}\right|-\left|F_{d}\right|+1\right) y_{u} \geq\left|F_{d}^{u}\right|
$$

One notable feature of the dynamic programming algorithm presented in the next section is that when $u=d$, the construction of inequality (5.4) does not require additional computational effort once we have solved for $F_{d}$. This feature stems from the fact that $F_{d}^{d}$ is readily available as a byproduct of the dynamic programming procedure.

\subsection{A Dynamic Programming Algorithm for Solving In-tree Subgraphs}

We employ a modification of the dynamic programming algorithm presented in Chapter 4 for solving tree networks. Let an in-tree corresponding to the destination node $d \in N$ be denoted by $T_{d}$. We define the level $\ell_{v}$ of node $v$ as the number of 
edges in the path from node $v$ to the root node $d\left(\ell_{d}=0\right)$. We let $S_{v}$ denote the children of node $v \in N$, which are the nodes on level $\ell_{v}+1$ that are adjacent to node $v$. The node at level $\ell_{v}-1$ that is adjacent to node $v$ is the parent of node $v$ and is denoted by $p^{(v)}$. We also define descendants of node $v$ to be a set of nodes $D_{v}, D_{v} \supseteq S_{v}$, such that a path from node $u \in D_{v}$ to the root node $d$ includes node $v$ as an intermediate node. Let $T_{v}$ be a subtree rooted at node $v$ induced by the node set $\{v\} \cup D_{v}$, and let $L_{v}$ be the set of leaf nodes in $T_{v}$.

Before presenting the details of the dynamic programming algorithm for solving in-tree networks, we first demonstrate how to calculate the traceback number with respect to node $d$. Accordingly, we describe a modification of the node aggregating procedure presented in Chapter 4, tailored to the in-tree problem encountered here.

Consider the network presented in Figure 5.1a in which the shaded nodes indicate filter placement. We aggregate contiguous unfiltered nodes into weighted nodes whose weights are equal to the number of aggregated nodes, and represent filtered leaf nodes with unit weighted nodes. Figure 5.1a shows sets of contiguous unfiltered nodes to be aggregated, and filtered leaf nodes, enclosed in dotted ellipses. The resulting weighted network after node aggregation is shown in Figure 5.1b. If we consider a given subtree $T_{v}$, for some $v \in N$, the root node $v$ will either be filtered or it will belong to an aggregated node in the corresponding aggregated network. In the latter case, we call the set of all unfiltered nodes included in the weighted node containing node $v$ an open set, denoted by $O_{v}$. If node $v$ is filtered, the open set for subtree $T_{v}$ is empty. The computation of the maximum traceback number in the aggregated network with respect to $d$ is reduced to finding a longest path (in terms of node weights) from a leaf node to the root node $d$. In our example in Figure 5.1, the longest path is highlighted by double edges in Figure 5.1c. From the total weight $w$ of a longest path to the root node $d$, we obtain the maximum traceback number as $w-\delta-1$, where $\delta=1$ if $\left|O_{d}\right|>0$, else, $\delta=0$.

We denote the set of all nodes that can successfully send packets to the root node $v$ of some subtree $T_{v}$ with purported origin address $i \in T_{v}$ under a given filter configuration $F$ by $N_{i v}(F)$. We also define a set $A_{v}$ related to a possible 


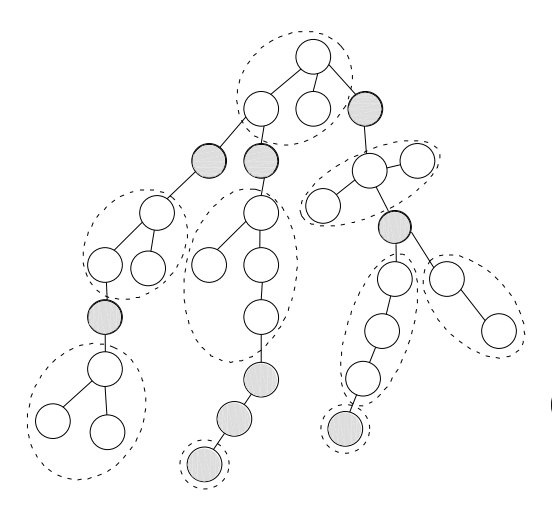

(a) Nodes to be aggregated.

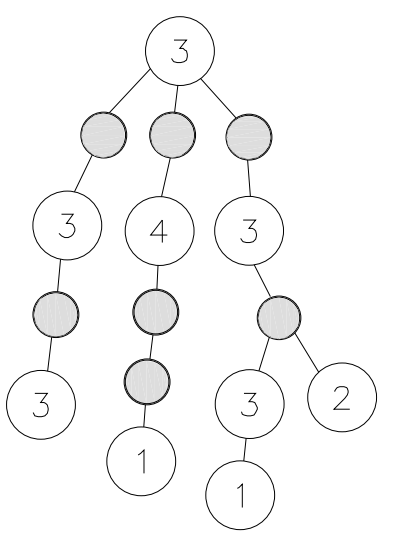

(b) Simplified network after node aggregation.

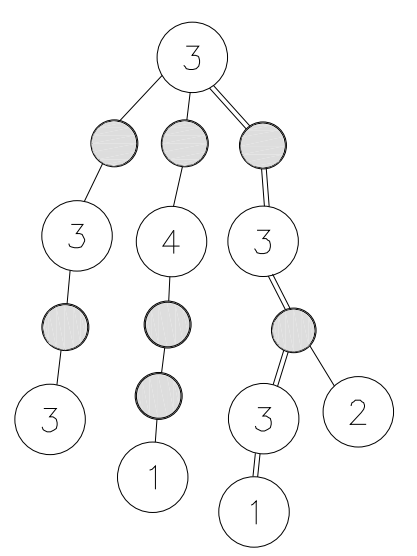

(c) Longest path highlighted with double edges.

Figure 5.1: The process of node aggregation for in-tree subgraphs.

attack on $v$ from a node within $T_{v}$ by $A_{v}=N_{\ell v}(F) \backslash\left(N_{\ell v}(F) \cap O_{v}\right)$, where $\ell \in$ $\operatorname{argmax}_{i \in L_{v}}\left\{\left|N_{i v}(F)\right|\right\}$. This is a largest set of nodes not in the open set $O_{v}$ that can send packets to the root node of the subtree $T_{v}$ with purported address of node $\ell \in L_{v}$. We refer to the cardinality of the set $A_{v}$ as the attack number. In the aggregated network, $A_{v}$ represents a longest weighted path from a leaf node (including the leaf node) to the aggregated cluster that includes node $v$, excluding 
the weight of the aggregated cluster containing $v$. At each stage of the procedure, we enforce the condition that $\left|A_{v}\right|+\left|O_{v}\right| \leq b+1$ if either $v \neq d$ or $O_{v}=0$, and otherwise, we require that $\left|A_{v}\right|+\left|O_{v}\right| \leq b+2$.

The algorithm starts from the leaf nodes and progresses systematically upward towards the root node $d$, creating a set of solutions for the subtree $T_{v}$, for some $v \in N$, from solutions of subtrees $T_{u}, \forall u \in S_{v}$. We keep pertinent features of candidate solutions to a subtree $T_{v}$ in an $\left(r_{v}+1\right) \times\left(c_{v}+1\right)$ matrix $B^{v}$, in which $0, \ldots, r_{v}=\left|D_{v}\right|-\left|L_{v}\right|+1$ represent the number of filters deployed in the subtree and the columns $0, \ldots, c_{v}=D_{v}+1$ represent the size of the open set for a candidate solution. An entry $b_{i j}^{v}$ of the matrix $B^{v}$ represents the minimum attack number for the subtree when $i$ filters are deployed so that the size of the open set is $j$. If $b_{i j}^{v}$ violates the traceback limit requirement, we set the corresponding matrix entry to infinity. The algorithm starts by analyzing base-case subtrees. A base-case subtree $T_{v}$ is a subtree that is either a maximal star or chain. Thus, $T_{v}$ is a base-case subtree if $S_{v}=L_{v}$ or if $T_{v}$ is a chain but $T_{p^{(v)}}$ is not. Column zero entries of the matrix $B^{v}$ corresponding to a chain base-case subtree are updated to $b_{i 0}^{v}=\left|D_{v}\right|-i$, for $i=1, \ldots,\left|D_{v}-1\right|$ such that $\left|D_{v}\right|-i-1 \leq b$. The entries corresponding to a positive open set for $i=1, \ldots,\left|D_{v}\right|-1$ are dominated by column zero entries and are thus left at infinity. The entry $b_{0\left|D_{v}\right|}^{v}$ is set to zero if $\left|D_{v}\right|-1 \leq b$.

We construct the matrix $B^{v}$ for the tree $T_{v}$ in two stages. In the first stage, we populate columns 1 through $c_{v}$ by allowing for positive open set sizes, and in the second stage we populate column 0 using a procedure that assumes the placement of a filter at node $v$. For base-case subtrees, the first stage involves updating the entry $b_{0\left|D_{v}\right|}^{v}$ to zero if $D_{v}-1 \leq b$, otherwise, we skip the first stage since the remaining entries with positive open set sizes are dominated by some column zero entry with fewer or the same number filters. In stage two of chain base-case subtrees, we update the entries $b_{i 0}^{v}$ to $\left|D_{v}\right|-i-1$ for $i=1, \ldots,\left|D_{v}\right|-1$ such that $\left|D_{v}\right|-1 \leq b$. For star base-case subtrees, stage two involves updating the entry $b_{10}^{v}$ to one; the rest of column zero entries will be dominated by this entry. After processing base-case subtrees, the algorithm proceeds to solve those subtrees $T_{v}$ such that all subtrees 
$T_{u}, \forall u \in S_{v}$, have already been solved.

We populate columns $1, \ldots, c_{v}$ through an intermediate procedure that utilizes a record-keeping scheme. Each record has four fields and is of the form $a=\left(a_{1}, a_{2}, a_{3}, a_{4}\right)$, where $a_{1}=\left|A_{v}\right|$ is the attack number, $a_{2}=\left|O_{v}\right|$ is the size of the open set, $a_{3}$ is the number of filters deployed in the subtree, and $a_{4}$ is the number of child subtrees used to obtain the current record. Since we update records by considering the addition of one child subtree solution at a time, we refer to $a_{4}$ as the stage of the intermediate procedure. For notational ease, we assume nodes in $S_{v}$ are labeled as $u_{1}, \ldots, u_{s_{v}}$, where $s_{v}=\left|S_{v}\right|$.

We create stage 1 records corresponding to each finite element of the solution matrix $B^{u_{1}}$ as follows: $a_{1}=b_{i j}^{u_{1}}, a_{2}=j+1, a_{3}=i$, and $a_{4}=1$. For a given traceback limit $b$, we only keep a record $a$ if $a_{1}+a_{2} \leq b+1$ (or $a_{1}+a_{2} \leq b+2$ if $v=d$ and $\left.a_{2}>0\right)$. Records in stage $k, k=2, \ldots s_{k}$, are computed by combining records from stage $k-1$ and finite elements from the solution matrix $B^{u_{k}}$. Let a record from stage $k$ be denoted by $a^{k}=\left(a_{1}^{k}, a_{2}^{k}, a_{3}^{k}, a_{4}^{k}\right)$. Then for each record previously recreated from stage $k-1$, and for each finite element $b_{i j}^{u_{k}}$ of $B^{u_{k}}$, we compute a stage $k$ record as follows: $a_{1}^{k}=\max \left\{a_{1}^{k-1}, b_{i j}^{u_{k}}\right\}, a_{2}^{k}=a_{2}^{k-1}+j, a_{3}^{k}=a_{3}^{k-1}+i$, and $a_{4}^{k}=k$. Of these records, we only keep feasible records that are not dominated by (or identical to) other records. A record $\tilde{a}$ dominates another record $\bar{a}$ if $\bar{a}_{1} \geq \tilde{a}_{1}, \bar{a}_{2} \geq \tilde{a}_{2}, \bar{a}_{3} \geq \tilde{a}_{3}$, and $\bar{a}_{4}=\tilde{a}_{4}$ with at least one strict inequality holding.

Once records for stage $s_{v}$ have been computed, we populate entries for the matrix $B^{v}$ in columns $1, \ldots, c_{v}$ corresponding to nondominated records of stage $s_{v}$. We also record the actual filter placement leading to each of these values. Initially, all entries of the matrix $B^{v}$ are initialized to infinity. From each nondominated record $a^{s_{v}}$, we obtain the matrix entry $b_{a_{3}^{s_{v}} a_{2}^{s_{v}}}^{v}=a_{1}^{s_{v}}$. The complexity for computing columns $1, \ldots, c_{v}$ for some $v \in N$ is $O\left(n^{4}\right)$ due to the required merging operation of $O\left(n^{2}\right)$ matrices (a similar argument for tree networks is made in Chapter 4).

We construct column 0 of the matrix $B^{v}$ through an intermediate $\mu_{v} \times s_{v}$ matrix $M$, where $\mu=\max _{j \in\left\{1, \ldots, s_{v}\right\}}\left\{r_{u_{j}}\right\}$, and $r_{u_{j}}$ is the minimum number of filters needed to ensure perfect security in a subtree $T_{u_{j}}$ (i.e., $r_{u_{j}}=\left|D_{u_{j}}\right|-\left|L_{u_{j}}\right|+1$ ). Initially, all 
entries of $M$ are set to infinity. We then compute finite entries of $M$ by iterating through each finite value $b_{i k}^{u_{j}}$ from the matrix $B^{u_{j}}$ and setting $m_{i j}=\min \left\{m_{i j}, b_{i k}^{u_{j}}+\right.$ $k\}$, for $i=0, \ldots, r_{u_{j}}$ and $j=1, \ldots, s_{u_{j}}$. If $r_{u_{j}}<\mu_{v}$ for some $j \in\left\{1, \ldots, s_{v}\right\}$, we set $m_{i j}=1$ for $i=r_{u_{j}}+1, \ldots, \mu_{v}$. The resulting matrix entries $m_{i j}$ represent the minimum attack number coming from the subtree $T_{u_{j}}$ if $i$ filters are deployed in $T_{u_{j}}$.

We say an element $m_{i j}$ of the matrix $M$ is covered if we have at least $i+1$ filters deployed in the subtree $T_{u_{j}}$; otherwise, the element is uncovered. We denote the largest uncovered element of $M$ by $\hat{m}$ and the corresponding column index by $\hat{\jmath}$. Solving for an element $b_{i 0}^{v}$ of the solution matrix $B^{v}$, for some $v \in N$, is equivalent to finding a distribution of $i-1$ filters amongst the columns of the matrix $M$ so that $\hat{m}$ is minimized. "(One filter is reserved for node $v$ itself since $O_{v}$ is assumed to be empty.) This objective can be achieved by greedily covering the largest element in $M$ until all allocated filters are exhausted. If the final attack number $\hat{m}$ is more than $b+1$ when using $i$ filters, we set $b_{i 0}^{v}=\infty$.

Once the matrix $B^{d}$ corresponding to the entire in-tree $T_{d}$, for some $d \in N$, has been populated, the minimum number of filters needed to achieve the level of security prescribed by $b$ is equal to the minimum row index of the matrix $B^{d}$ that contains a finite entry. After identifying the minimum number of filters required, we reconstruct the solution by backtracking through the subtrees from the root node down to the base-case subtrees.

The worst-case complexity for computing column 0 of the solution matrix $B^{v}$, for some $v \in N$, is $O\left(n^{2}\right)$, due to the process of populating the $M$ matrix from the $B$ matrices. Therefore, the computational complexity for solving all columns 0 of for the entire in-tree is $O\left(n^{3}\right)$. Thus, the overall complexity for solving an in-tree is $O\left(n^{5}\right)$, because of the bottleneck operation required to fill columns $1, \ldots, c_{v}$ of $B^{v}$ matrices for $v \in N$. 


\subsection{Heuristic Algorithms}

We provide three heuristics for solving the GMFPP in this section. In Section 5.3.1 we discuss a linear programming-based heuristic. A heuristic based on the dynamic programming algorithm for solving tree networks given in Chapter 4 is presented in Section 5.3.2, and finally a greedy heuristic that utilizes network routing information to estimate the optimal solution to the GMFPP is discussed in Section 5.3.3.

\subsubsection{LP-based Heuristic}

First, we prescribe a standard rounding heuristic based on solving the linear relaxation of (3.1). Given a solution $\hat{y}=\left(\hat{y}_{1}, \ldots, \hat{y}_{n}\right)$ of the linear relaxation of $(3.1)$, define $I_{f}$ to be a set of indices of all fractional components of this solution vector. If $I_{f}=\emptyset$, we return the solution $\hat{y}$ as the heuristic solution. Else, we fix $\hat{y}_{m}=1$, where $m \in \operatorname{argmax}_{i \in I_{f}}\left\{\hat{y}_{i}\right\}$, in the linear programming relaxation and resolve the resulting linear program. We repeat the process until $I_{f}=\emptyset$, after which we have obtained an integer feasible solution $\hat{y}$ to (3.1). Figure 5.2 summarizes the LP-based heuristic.

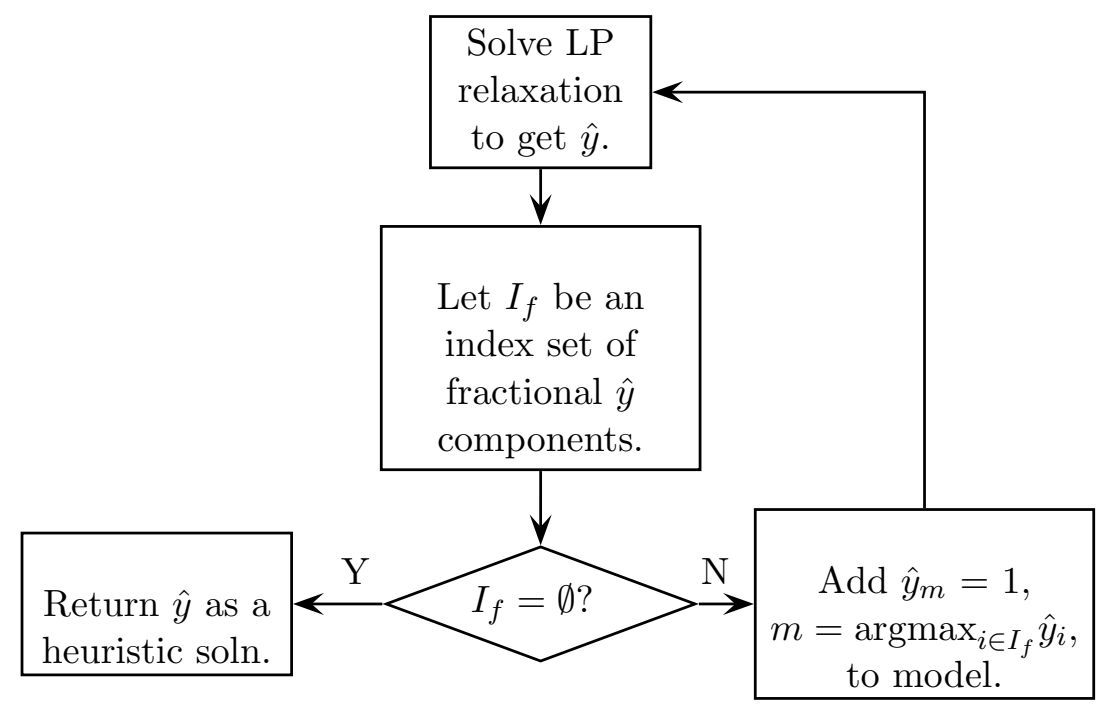

Figure 5.2: Schematic of the LP-based heuristic. 


\subsubsection{Dynamic Programming-based Heuristic}

In this section we describe a heuristic based on the dynamic programming algorithm for solving the GMFPP on tree networks. The heuristic is based on an assumption that if we consider only the routing of packets to a node $d \in N$, from all other nodes in the set $N \backslash\{d\}$, we obtain a directed in-tree rooted at node $d$. We can approach a heuristic for communication networks satisfying this requirement by iteratively solving in-tree networks corresponding to each destination node $d \in N$.

This heuristic utilizes the dynamic programming algorithm for solving in-trees given in the appendix to estimate the solution to a GMFPP. (The details of this algorithm can be skipped on a casual reading of this section.) We describe three variations of the dynamic programming-based heuristic.

In the first variation, which we call DP1, the heuristic proceeds as follows. We initialize the heuristic solution $\bar{F}=\emptyset$. For each node $d \in N$, we solve the GMFPP on the in-tree $T_{d}$ to obtain an optimal solution $F_{d}$. For each node $v \in N$, let $n_{v}$ denote the number of solutions $F_{d}, d \in N$, in which node $v$ appears. We add node $\bar{v} \in \operatorname{argmax}_{v \in(N \backslash \bar{F})}\left\{n_{v}\right\}$ to the solution $\bar{F}$. We then resolve all the in-trees $T_{d}$ such that $\bar{v} \notin F_{d}$ with the stipulation that all the nodes in the set $\bar{F}$ are already filtered. We repeat this process until $\bar{F}$ is a feasible solution to each in-tree $T_{d}, \forall d \in N$. At this point, we return $\bar{F}$ as the heuristic solution. The DP1 heuristic is summarized in Figure 5.3.

The second version (DP2) differs from DP1 in the way we pick nodes to be included in the heuristic solution. The motivation behind this heuristic is that several optimal solutions may exist to each in-tree. Hence, it might be more appropriate to select a node to belong to $\bar{F}$ that appears in some alternative optimal solution. We again initialize the heuristic solution $\bar{F}=\emptyset$ and solve all in-trees $T_{d}, \forall d \in N$, to obtain corresponding solutions $F_{d}$. For each node $u \in N \backslash \bar{F}$, we resolve all in-trees $T_{d}, d \in N$, such that $u \notin F_{d}$ subject to the condition that node $u$ is filtered. Let $F_{d}^{u}$ denote the new optimal solution for the in-tree $T_{d}$ (note that $\left|F_{d}^{u}\right| \geq\left|F_{d}\right|$ ). Let $\tilde{n}_{u}$ be the number of in-trees $T_{d}, d \in N$, such that $\left|F_{d}^{u}\right|=\left|F_{d}\right|$, plus the number of 


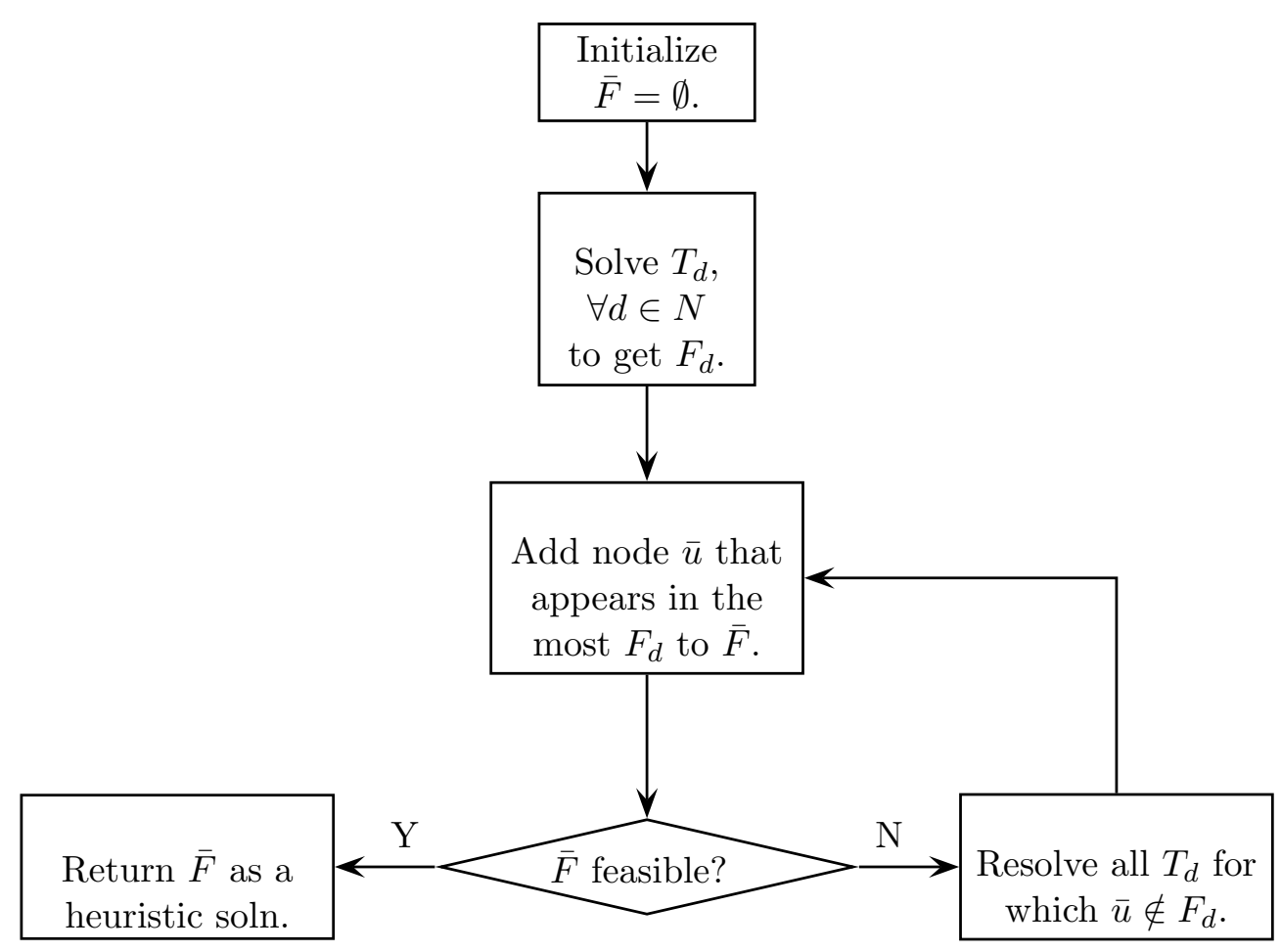

Figure 5.3: Schematic of the DP1 heuristic.

those in-trees for which $u \in F_{d}$; the value $\tilde{n}_{u}$ now represents the number of solutions in which $u$ is filtered in at least one alternative optimal solution. We add a node $\bar{u} \in \operatorname{argmax}_{u \in N \backslash \bar{F}}\left\{\tilde{n}_{u}\right\}$ to the heuristic solution $\bar{F}$, and then proceed as in DP1. A schematic representation of DP2 is shown in Figure 5.4

The third version of the heuristic (DP3) is an extension of DP2 through a two-opt post-processing of the solution. The first stage of post-processing checks whether removing any node $v \in \bar{F}$ will result in a feasible solution. If for some $v \in \bar{F}$, we have that $\bar{F} \backslash\{v\}$ is feasible, we update $\bar{F}=\bar{F} \backslash\{v\}$. In the second stage of post-processing, we examine possible swaps in which a node $i \in \bar{F}$ is swapped with a node $j \in N \backslash \bar{F}$. Let $\bar{F}_{a}$ be the solution we obtain by executing such a swap. If $\bar{F}_{a}$ is not feasible, we "reject" $\bar{F}_{a}$ and revert to $\bar{F}$. Also, if $\bar{F}_{a}$ is feasible, but phase one post-processing indicates that no filter in $\bar{F}_{a}$ can feasibly be removed, we also 




Figure 5.4: Schematic of the DP2 heuristic.

reject $\bar{F}_{a}$. Else, we identify a node $v \in \bar{F}_{a}$ that can feasibly be removed from $\bar{F}_{a}$, update $\bar{F}=\bar{F}_{a} \backslash\{v\}$, and restart the second phase of postprocessing. We continue with this procedure until all node pair swaps from the current solution $\bar{F}$ have been rejected.

\subsubsection{Greedy Multiple Restart Heuristic}

The heuristic we present in this section uses routing information to construct an auxiliary graph $\bar{G}=(\bar{N}, \bar{E})$, where $|\bar{N}|=n+n(n-1)+n(n-1)(n-2)$. For ease of notation and presentation, we partition the node set $\bar{N}$ into three subsets, $\bar{N}_{1}$, $\bar{N}_{2}$, and $\bar{N}_{3}$, with $\left|\bar{N}_{1}\right|=n,\left|\bar{N}_{2}\right|=n(n-1)$ and $\left|\bar{N}_{3}\right|=n(n-1)(n-2)$. Nodes in the set $\bar{N}_{1}$ represent the original nodes in the communication network. Each node in $\bar{N}_{2}$ represents a forged address/victim pair $(o, d) \in C$, and a node in $\bar{N}_{3}$ represents an attack on node $d \in N$ from node $s \in N \backslash\{d\}$ with purported origin 
address $o \in N \backslash\{s, d\}$, where $(s, d) \in C$ and $o \in N \backslash\{s, d\}$ such that $(o, d) \in C$. We partition the edge set $\bar{E}$ into sets $\bar{E}_{1}$ and $\bar{E}_{2}$. We construct an edge $(i, j) \in \bar{E}_{1}$ if and only if putting a filter on node $i \in \bar{N}_{1}$ can stop an attack represented by node $j \in \bar{N}_{3}$. Edges in $\bar{E}_{2}$ are constructed between each node $k \in \bar{N}_{2}$ representing a forged address/victim pair $(o, d)$ and all nodes $j \in \bar{N}_{3}$ representing an attack on node $d$ with forged address $o$ by node $s, \forall s \in \bar{N}_{1} \backslash\{o, d\}$.

The graph $\bar{G}=(\bar{N}, \bar{E})$ relates to the GMFPP as follows. The degree of each node $v \in \bar{N}_{1}$ represents the number of attacks that can be stopped by placing a filter on node $v$. The set of all nodes in $\bar{N}_{1}$ that are adjacent to a node $v \in \bar{N}_{3}$ is the $G_{\text {sod }}$ set for the attack represented by node $v$. The degree of a node $v \in \bar{N}_{2}$ can be interpreted as the traceback number of the $(o, d)$ pair that node $v$ represents.

For a given traceback limit $0 \leq b \leq n-3$, the heuristic proceeds as follows. We initialize the solution set $F=\emptyset$. Assume that nodes in $\bar{N}_{1}$ are labeled $1, \ldots, n$. We designate node 1 as the initial node and add it to $F$. Delete node 1 and all nodes $j \in \bar{N}_{3}$ adjacent to it, and update the edge sets $\bar{E}_{1}$ and $\bar{E}_{2}$ by deleting edges that were incident to the deleted nodes. We then scan the nodes in $\bar{N}_{2}$ and delete all those nodes that have degree less than or equal to the traceback limit $b$. This in turn allows us to remove all nodes in $\bar{N}_{3}$ that were adjacent to the deleted nodes in $\bar{N}_{2}$, updating the edge sets $\bar{E}_{1}$ and $\bar{E}_{2}$ accordingly. If the node set $\bar{N}_{2}$ is empty, we terminate with $F$ as an incumbent solution $\bar{F}$. Otherwise, we find a node in $\bar{N}_{1}$ that has the highest degree and add it to $F$, and repeat as above until $\bar{N}_{2}$ is empty, at which point we terminate with an incumbent $\bar{F}=F$. We then resolve the problem using node $j, j=2, \ldots, n$, as the initial node, each time updating the incumbent solution $\bar{F}$ to $F$ if $|\bar{F}|>|F|$. We return $\bar{F}$ as the heuristic solution.

Restarting the procedure using a different initial node each time increases the chances of getting a good solution. These chances can even be improved further by designating $k \geq 2$ initial nodes instead of a single initial node. If we use $k$ initial nodes, we need to restart the procedure $\left(\begin{array}{l}n \\ k\end{array}\right)$ times corresponding to the $k$ node distinct subsets of $\bar{N}_{1}$. In this study, we explored the use of $k=0,1,2$ as the size of the designated initial solution. (Solving the GMFPP with $k=0$ corresponds 
to solving the heuristic only once using a maximum degree node in $\bar{N}_{1}$ as the initial node.)

\subsection{Computational Tests}

In this section, we present experimental results to evaluate the effectiveness of the valid inequalities discussed in this chapter. We also evaluate the performance of proposed heuristics against the optimal solutions of the mixed-integer programming model. We briefly discuss the implementation of programs and the generation of test instances in Section 5.4.1, followed by our computational results in Section 5.4.2

\subsubsection{Generation of Instances}

We generated two series of instance sets. In the first series, we randomly generated six sets of problem instances to evaluate the effectiveness of valid inequalities and the performance of heuristic algorithms. Ten instances were generated for each instance set, for a total of 60 instances. We refer to an instance set containing instances with $n$ nodes as "Inst_n." Using this nomenclature, the six instance sets we generated were Inst_15, Inst_20, Inst_25, Inst_30, Inst_35, and Inst_40.

For each instance, we generated an undirected graph with an edge density of 0.4. This was accomplished by generating a uniform random number $U(0,1)$ for each distinct node pair $(i, j), i \in N$ and $j \in N \backslash\{i\}$. The edge $(i, j)$ was constructed if and only if the generated random number was at most 0.4. A single routing path between every distinct node pair was generated for each test instance, i.e., $C$ is complete and $|R(o, d)|=1$ for all $o \in N, d \in N, o \neq d$. The routing information was obtained by randomly generating a positive weight for each edge $(i, j) \in E$. The routing of packets to a given node $d \in N$ from all other nodes in the set $N \backslash\{d\}$ was conducted along shortest paths in terms of the edge weights. (Since floating-point numbers were used for edge weights, ties in shortest paths were not encountered.) The shortest path routing policy ensured that the routing of packets to a node $d \in N$ from all other nodes form an in-tree rooted at node $d$, as required by the dynamic 
programming heuristic.

The second series of instance sets was aimed at evaluating the effectiveness of valid inequalities from independent subgraphs at high traceback values since the randomly generated instances from the first series did not contain large enough independent subgraphs. For instance, the highest traceback number for which could construct chain and star inequalities was eight, and zero for clique inequalities. These instance sets were generated by starting with a backbone network consisting of the relevant independent subgraphs (chain, star, or clique). We first generated a 20-node backbone and then added 10 nodes to the network linked by a set of randomly generated edges using an edge density of 0.5 . The communication of each of the 10 additional nodes with all other nodes was conducted according to the shortest path routing policy we employed in the first series of instance sets. Each instance set had five instances for each of the three independent subgraphs for a total of 15 instances. We refer to an instance with $n$ total nodes whose backbone network has $m$ nodes as Inst_n_ind_m.

The identification of independent star subgraphs was implemented according to the following procedure. For each node $v \in N$, define $H_{v}$ to be the nodes adjacent to $v$. We initialize the set of leaf nodes $N_{v}$ that belong to the final independent star subgraph to be the empty set. The main step proceeds as follows. Create a set $S$ consisting of all $(o, d) \in C$ that meet the following requirements: (a) $o \in H_{v}-N_{v}$ and $d \in H_{v}-N_{v}$, (b) $R(o, d)=\{o, v, d\}$, and (c) for all $k \in N_{v}$, both $R(o, k)=\{o, v, k\}$ and $R(d, k)=\{d, v, k\}$. If $S \neq \emptyset$, then determine a node $\hat{\imath}$ that appears in the most pairs in $S$, add $\hat{\imath}$ to $N_{v}$, and repeat the main step. Else, determine if there exists a node $o \in H_{v}-N_{v}$ such that $R(o, k)=\{o, v, k\}$ for all $k \in H_{v}$. If so, add $o$ to $N_{v}$ and terminate; else, simply terminate the algorithm. If $\left|N_{v}\right| \geq b+2$ then a valid inequality can be added based on the independent star centered at node $v$ with leaf nodes $N_{v}$.

Independent chain subgraphs were identified by simply inspecting each path in the set of routes. Routing among nodes in any path $p$ is implied by the shortest path routing policy, and thus the chain represented by this path is indeed independent. 
If $|N(p)| \geq b+3$, an inequality corresponding to the independent chain represented by the path $p$ was generated.

Since the problem of identifying a maximum clique in a graph is NP-hard [31], we employed the following heuristic to identify independent cliques. For a given traceback number $b$, we identified a set of nodes $Q$ such that a node $v \in Q$ if and only if $v$ communicates with at least $b+3$ nodes along single-arc paths. Denote $\mathcal{K}$ as the set of nodes that belong to the final independent clique ( $\mathcal{K}$ is initially empty). The main step proceeds as follows. Select a node $\tilde{v}$ from the set $Q$ that meets the following two criteria: (1) $\tilde{v}$ communicates with each of the nodes in the set $\mathcal{K}$ along a single-arc path, and (2) $\tilde{v}$ communicates with the most number of nodes in $Q$ along single-arc paths. We update the sets $\mathcal{K}=\mathcal{K} \cup\{\tilde{v}\}$ and $Q=Q \backslash\{\tilde{v}\}$. We repeat the main step until either one of the following two cases happens: (1) $Q$ is empty, in which case we construct an inequality from the independent clique $\mathcal{K},(2)$ we cannot find a $\tilde{v}$ that routes to all nodes in $\mathcal{K}$ along a single-arc path. In this case we construct an inequality if $|\mathcal{K}| \geq b+3$, otherwise we try to find a different clique by reinitializing $\mathcal{K}$ with a node from $Q$ that does not belong to the current clique, and proceeding according to the main step.

\subsubsection{Computational Results}

We present computational results for the mixed-integer programming model and evaluate the performance of the heuristic algorithms presented in Section 5.3 against the mixed-integer programming solutions. All computational results presented in this section assume the use of maximal filters. Each program was allowed a maximum of three hours per problem instance after which the program was terminated.

Tables 5.1-5.6 show the effect of adding chain and star inequalities to the mixedinteger programming model on the computational times. (For these instances, it is impractical to analyze the impact of clique inequalities, because it is very unlikely that cliques exist in the graph that are large enough to give rise to a nontrivial inequality.) The column labeled "MIP" shows the average CPU times of the mixedinteger programming model with no cuts, while the columns labeled "Chain Cuts", 
"Star Cuts", and "Combined" show the average CPU times when the chain inequalities, the star inequalities, and both the chain and star inequalities were added to the mixed-integer programming model, respectively. The subcolumn labeled "Count" shows the number of instances out of ten for which corresponding independent subgraphs were identified that were large enough to warrant the construction of nontrivial associated valid inequalities. The MIP CPU times were averaged only over the same instances for which the inequalities were generated. Blank entries in the tables represent cases for which the node set of the largest independent subgraph identified over the ten instances had a cardinality less than $b+3$, and thus we could not generate nontrivial inequalities for the entire instance set at the corresponding traceback limit.

Tables 5.1-5.6 demonstrate that the chain inequalities actually hinder the progress of the CPLEX branch-and-bound solver when $b=1$, and otherwise provide inconsistent results. The star inequalities serve to slightly improve the solvability of the mixed-integer programming model, but only for the smaller values of $b$ for which such inequalities could be found. We also attempted to limit chain inequalities to the $k$ biggest chains for different values of $k$, and to use these inequalities in a cutting-plane fashion. However, none of these strategies appears to provide any advantage over the use of star inequalities alone.

One reason for the lack of effectiveness of these cuts is that the subgraphs generated are small portions of the overall network. By contrast, these subgraphs are much more prominent in our Inst_30_ind_20 instances. For this case, Table 5.7 demonstrates that the chain inequalities now provide a modest computational benefit (an average CPU reduction of about 5\%), and the star inequalities reduce the average CPU time by about $38 \%$. The clique inequalities are extremely effective for relatively small values of $b$ (in this case, $b \leq 4$ ), but no clear pattern exists for larger values of $b$.

Tables 5.8-5.13 show that the average CPU times increased with the addition of dynamic programming-based inequalities. The increase in the average CPU times is more prominent at larger traceback limits relative to the instance size. This can 
be attributed to the architecture of the dynamic programming algorithm coupled with the increase in the size of the mixed-integer programming model due to the addition of inequalities. As the traceback limit increases, the dynamic programming algorithm's computational complexity approaches the theoretical bound of $O\left(n^{5}\right)$; hence, it becomes relatively expensive to construct the corresponding inequalities. In addition, we are typically adding on the order of $O(n)$ inequalities in the vicinity of the linear relaxation optimal solution. This increases the number of extreme points that the linear programming solver has to traverse before finding an optimal solution. The results imply that the amount of tightening of the linear relaxation feasible region we obtain by the addition of dynamic programming-based inequalities is not enough to offset the required increase in computational effort.

Heuristic results are presented in Tables 5.14-5.20. Tables 5.14-5.19 show the suboptimality of each heuristic algorithm at varied traceback limits for each of the six instance sets Inst_15-Inst_40. The suboptimality of a heuristic solution is defined as the difference between the heuristic objective value and the optimal objective value. Thus, the columns under the heading "Total Suboptimality" show the sum of differences between the heuristic objective value and the optimal mixed-integer programming objective value over the 10 instances in each instance set at the specified traceback limits. The columns under the heading "Maximum Suboptimality" show the corresponding maximum difference. Individual heuristic results are shown in the subcolumns labeled "LP" for the linear programming-based heuristic, "DP1", "DP2", and "DP3" for the three versions of the dynamic programming-based heuristics, and "MRGH" for multiple restart greedy heuristic. The MRGH has three subcolumns labeled with the size of the initial solution used. The results show that the multiple restart greedy heuristic with $k=2$ outperformed the other heuristics in terms of solution quality; however, it was computationally very expensive relative to the other heuristics. DP3 performed better than the LP-based heuristic both in terms of solution quality and the amount of average computational time expended. DP3 also performed better than the multiple restart greedy heuristic at $k=1$ in terms of solution quality although it took about twice the CPU time on 
average. Another observation from Tables 5.14-5.19 is the amount of improvement achieved by the post-processing of DP2 solutions (given by the difference between the DP3 total suboptimality and the corresponding DP2 total suboptimality). The results show that, on average, post-processing of DP2 solutions reduced the total suboptimality by more than half. Table 5.20 shows the average CPU seconds each algorithm took to solve an instance from each of the six instance sets. The computational times were averaged over the $10(n-2)$ combinations of instance topology and traceback limit (each of the ten instances solved using a different traceback limit $b=0, \ldots, n-3)$.

Figures 5.5-5.7 show the relationship between the total suboptimality and the CPU time for the heuristic procedures for the instance sets Inst_20, Inst_30, and Inst_40. These figures depict the tradeoff observed in heuristic execution time and total suboptimality, with the exception of the LP-based heuristic. The LP-based heuristic consumed more CPU time than did the DP3, MRGH with $k=1$, and DP2 heuristics but yielded higher total suboptimality. 
Table 5.1: Average CPU times for Inst_15 in seconds.

\begin{tabular}{|c|c|c|c||c|c|c||c|c|c|}
\hline$b$ & MIP & \multicolumn{2}{|c||}{ Chain Cuts } & MIP & \multicolumn{2}{c||}{ Star Cuts } & \multicolumn{2}{c|}{ MIP } & \multicolumn{2}{c|}{ Combined } \\
\cline { 2 - 9 } & CPU & CPU & Count & CPU & CPU & Count & CPU & CPU & Count \\
\hline 0 & 0.20 & 0.21 & 10 & 0.20 & 0.20 & 10 & 0.20 & 0.19 & 10 \\
1 & 0.48 & 0.61 & 10 & 0.48 & 0.49 & 10 & 0.48 & 0.64 & 10 \\
2 & 0.64 & 0.70 & 10 & 0.51 & 0.55 & 9 & 0.64 & 0.69 & 10 \\
3 & 1.02 & 1.12 & 9 & 0.82 & 0.71 & 5 & 1.00 & 1.06 & 10 \\
4 & 0.69 & 0.67 & 3 & & & & 0.69 & 0.69 & 3 \\
\hline
\end{tabular}

Table 5.2: Average CPU times for Inst_20 in seconds.

\begin{tabular}{|c|c|c|c||c|c|c||c|c|c|}
\hline$b$ & MIP & \multicolumn{2}{|c||}{ Chain Cuts } & MIP & \multicolumn{2}{c||}{ Star Cuts } & \multicolumn{2}{c|}{ MIP } & \multicolumn{2}{c|}{ Combined } \\
\cline { 2 - 9 } & CPU & CPU & Count & CPU & CPU & Count & CPU & CPU & Count \\
\hline 0 & 0.47 & 0.45 & 10 & 0.47 & 0.46 & 10 & 0.47 & 0.44 & 10 \\
1 & 2.15 & 2.92 & 10 & 2.15 & 1.87 & 10 & 2.15 & 3.08 & 10 \\
2 & 4.04 & 3.73 & 10 & 3.89 & 3.71 & 9 & 4.04 & 3.53 & 10 \\
3 & 4.34 & 3.97 & 10 & 4.70 & 4.14 & 5 & 4.34 & 3.71 & 10 \\
4 & 5.30 & 5.68 & 8 & 2.30 & 2.00 & 1 & 5.30 & 5.51 & 8 \\
5 & 4.18 & 3.99 & 5 & & & & 4.18 & 3.99 & 5 \\
6 & 3.90 & 4.25 & 2 & & & & 3.90 & 4.28 & 2 \\
7 & 6.74 & 8.82 & 1 & & & & 6.74 & 8.90 & 1 \\
8 & 7.01 & 6.28 & 1 & & & & 7.01 & 6.34 & 1 \\
\hline
\end{tabular}

Table 5.3: Average CPU times for Inst_25 in seconds.

\begin{tabular}{|c|c|c|c||c|c|c||c|c|c|}
\hline$b$ & MIP & \multicolumn{2}{|c||}{ Chain Cuts } & MIP & \multicolumn{2}{c||}{ Star Cuts } & \multicolumn{2}{c|}{ MIP } & \multicolumn{2}{c|}{ Combined } \\
\cline { 2 - 9 } & CPU & CPU & Count & CPU & CPU & Count & CPU & CPU & Count \\
\hline 0 & 0.91 & 0.91 & 10 & 0.91 & 0.92 & 10 & 0.91 & 0.90 & 10 \\
1 & 16.61 & 18.72 & 10 & 16.61 & 9.62 & 10 & 16.61 & 14.23 & 10 \\
2 & 44.63 & 43.79 & 10 & 44.63 & 44.23 & 10 & 44.63 & 44.81 & 10 \\
3 & 70.27 & 64.85 & 10 & 74.86 & 66.93 & 9 & 70.27 & 63.33 & 10 \\
4 & 75.19 & 76.68 & 7 & 66.14 & 65.76 & 4 & 78.47 & 79.47 & 9 \\
5 & 87.81 & 86.77 & 2 & 49.72 & 50.49 & 2 & 68.76 & 88.20 & 4 \\
\hline
\end{tabular}


Table 5.4: Average CPU times for Inst_30 in seconds.

\begin{tabular}{|c|c|c|c||c|c|c||c|c|c|}
\hline$b$ & MIP & \multicolumn{2}{|c||}{ Chain Cuts } & MIP & \multicolumn{2}{c||}{ Star Cuts } & \multicolumn{2}{c|}{ MIP } & \multicolumn{2}{c|}{ Combined } \\
\cline { 2 - 9 } & CPU & CPU & Count & CPU & CPU & Count & CPU & CPU & Count \\
\hline 0 & 1.65 & 1.62 & 10 & 1.65 & 1.67 & 10 & 1.65 & 1.62 & 10 \\
1 & 28.84 & 45.80 & 10 & 28.84 & 23.12 & 10 & 28.84 & 38.21 & 10 \\
2 & 107.06 & 105.48 & 10 & 107.06 & 95.76 & 10 & 107.06 & 103.21 & 10 \\
3 & 206.80 & 196.86 & 10 & 206.80 & 206.60 & 10 & 206.80 & 212.06 & 10 \\
4 & 219.88 & 221.85 & 8 & 249.98 & 265.41 & 5 & 246.73 & 226.46 & 9 \\
5 & 39.86 & 39.33 & 3 & & & & 39.86 & 39.97 & 3 \\
6 & 55.54 & 54.58 & 3 & & & & 55.54 & 55.38 & 3 \\
7 & 111.07 & 100.74 & 1 & & & & 111.07 & 100.98 & 1 \\
8 & 59.35 & 54.55 & 1 & & & & 59.35 & 55.04 & 1 \\
\hline
\end{tabular}

Table 5.5: Average CPU times for Inst_35 in seconds.

\begin{tabular}{|c|c|c|c||c|c|c||c|c|c|}
\hline$b$ & MIP & \multicolumn{2}{|c|}{ Chain Cuts } & MIP & \multicolumn{2}{c||}{ Star Cuts } & MIP & \multicolumn{2}{c|}{ Combined } \\
\cline { 2 - 9 } & CPU & CPU & Count & CPU & CPU & Count & CPU & CPU & Count \\
\hline 0 & 2.74 & 2.68 & 10 & 2.74 & 2.78 & 10 & 2.74 & 2.71 & 10 \\
1 & 81.38 & 163.12 & 10 & 81.38 & 66.31 & 10 & 81.38 & 132.56 & 10 \\
2 & 302.97 & 285.38 & 10 & 302.97 & 300.35 & 10 & 302.97 & 316.56 & 10 \\
3 & 596.81 & 720.90 & 10 & 596.81 & 594.71 & 10 & 596.81 & 581.73 & 10 \\
4 & 1014.15 & 960.81 & 10 & & & & 1014.15 & 1016.99 & 10 \\
5 & 884.52 & 952.40 & 8 & & & & 884.52 & 974.01 & 8 \\
6 & 600.28 & 510.10 & 5 & & & & 600.28 & 513.53 & 5 \\
7 & 776.71 & 772.36 & 2 & & & & 776.71 & 783.87 & 2 \\
\hline
\end{tabular}


Table 5.6: Average CPU times for Inst_40 in seconds.

\begin{tabular}{|c|c|c|c||c|c|c||c|c|c|}
\hline$b$ & MIP & \multicolumn{2}{|c||}{ Chain Cuts } & MIP & \multicolumn{2}{c||}{ Star Cuts } & MIP & \multicolumn{2}{c|}{ Combined } \\
\cline { 2 - 9 } & CPU & CPU & Count & CPU & CPU & Count & CPU & CPU & Count \\
\hline 0 & 4.31 & 4.17 & 10 & 4.31 & 4.36 & 10 & 4.31 & 4.14 & 10 \\
1 & 165.05 & 375.35 & 10 & 165.05 & 127.35 & 10 & 165.05 & 278.37 & 10 \\
2 & 366.65 & 314.04 & 10 & 366.65 & 326.35 & 10 & 366.65 & 310.44 & 10 \\
3 & 1094.22 & 1167.51 & 10 & 1094.22 & 1101.72 & 10 & 1094.22 & 994.98 & 10 \\
4 & 1916.04 & 1910.23 & 10 & 576.87 & 514.01 & 2 & 1916.04 & 2046.26 & 10 \\
5 & 2603.60 & 2166.27 & 10 & & & & 2603.60 & 2185.18 & 10 \\
6 & 1113.29 & 1614.25 & 7 & & & & 1113.29 & 1654.53 & 7 \\
7 & 1634.21 & 2786.12 & 4 & & & & 1634.21 & 2810.22 & 4 \\
8 & 833.67 & 839.04 & 1 & & & & 833.67 & 848.98 & 1 \\
\hline
\end{tabular}

Table 5.7: Average CPU times for Inst_30_ind_20 in seconds.

\begin{tabular}{|c|c|c||c|c||c|c|}
\hline$b$ & \multicolumn{2}{|c||}{ Chain Backbone } & \multicolumn{2}{c||}{ Star Backbone } & \multicolumn{2}{c|}{ Clique Backbone } \\
\cline { 2 - 7 } & No Cuts & With Cuts & No Cuts & With Cuts & No Cuts & With Cuts \\
\hline 0 & 1.83 & 1.95 & 1.63 & 1.76 & 1.65 & 1.79 \\
1 & 22.96 & 23.33 & 8.65 & 8.67 & 194.52 & 16.12 \\
2 & 10.32 & 9.06 & 31.35 & 23.35 & 401.52 & 30.51 \\
3 & 25.01 & 12.17 & 40.38 & 35.20 & 536.82 & 109.27 \\
4 & 20.25 & 22.37 & 47.88 & 22.74 & 612.26 & 260.56 \\
5 & 37.97 & 32.90 & 72.80 & 41.00 & 925.39 & 961.75 \\
6 & 62.01 & 68.09 & 83.98 & 55.92 & 1350.49 & 647.18 \\
7 & 68.63 & 67.08 & 92.53 & 57.36 & 765.65 & 947.95 \\
8 & 120.50 & 109.32 & 95.92 & 79.96 & 1089.43 & 1234.84 \\
9 & 178.18 & 136.20 & 79.23 & 32.16 & 1470.90 & 1142.50 \\
10 & 213.72 & 197.20 & 79.51 & 36.14 & 2474.19 & 1397.30 \\
11 & 201.01 & 248.62 & 85.64 & 61.38 & 1698.89 & 1215.40 \\
12 & 331.33 & 370.07 & 67.12 & 28.69 & 1086.68 & 1466.06 \\
13 & 446.51 & 368.31 & 73.32 & 49.45 & 1208.74 & 1020.44 \\
14 & 570.77 & 505.44 & 71.92 & 27.48 & 1574.81 & 2049.90 \\
15 & 379.00 & 415.10 & 42.94 & 16.19 & 912.72 & 1097.82 \\
16 & 408.67 & 372.27 & 55.73 & 33.45 & 620.02 & 771.62 \\
17 & 255.42 & 209.53 & 43.47 & 48.92 & 835.59 & 873.19 \\
\hline
\end{tabular}


Table 5.8: Average CPU seconds for Inst_15 with DP inequalities.

\begin{tabular}{|c|c|c||c|c|c|}
\hline \multirow{3}{*}{$b$} & \multicolumn{2}{|c||}{ Average CPU } & & \multicolumn{2}{|c|}{ Average CPU } \\
\cline { 2 - 3 } \cline { 5 - 5 } & MIP No Cuts & DP Cuts & & MIP No Cuts & DP Cuts \\
\hline 1 & 0.48 & 0.52 & 7 & 1.12 & 1.49 \\
2 & 0.64 & 0.79 & 8 & 1.08 & 1.54 \\
3 & 1.00 & 1.25 & 9 & 0.65 & 0.79 \\
4 & 1.03 & 1.46 & 10 & 0.52 & 1.07 \\
5 & 0.91 & 1.44 & 11 & 0.51 & 2.04 \\
6 & 1.05 & 1.96 & 12 & 0.50 & 1.84 \\
\hline
\end{tabular}

Table 5.9: Average CPU seconds for Inst_20 with DP inequalities.

\begin{tabular}{|c|c|c||c|c|c|}
\hline \multirow{3}{*}{$b$} & \multicolumn{2}{|c||}{ Average CPU } & & \multicolumn{2}{|c|}{ Average CPU } \\
\cline { 2 - 5 } \cline { 4 - 5 } & MIP No Cuts & DP Cuts & $b$ & MIP No Cuts & DP Cuts \\
\hline 1 & 2.2 & 2.3 & 10 & 5.5 & 9.5 \\
2 & 4.0 & 4.1 & 11 & 5.6 & 6.4 \\
3 & 4.3 & 4.5 & 12 & 2.9 & 4.1 \\
4 & 6.0 & 7.6 & 13 & 4.2 & 6.7 \\
5 & 6.0 & 6.8 & 14 & 4.2 & 7.7 \\
6 & 4.7 & 6.9 & 15 & 1.5 & 16.4 \\
7 & 7.6 & 11.4 & 16 & 2.1 & 15.7 \\
8 & 5.2 & 8.2 & 17 & 1.6 & 14.3 \\
9 & 5.6 & 11.0 & & & \\
\hline
\end{tabular}


Table 5.10: Average CPU seconds for Inst_25 with DP inequalities.

\begin{tabular}{|c|c|c||c|c|c|}
\hline \multirow{2}{*}{$b$} & \multicolumn{2}{|c||}{ Average CPU } & & \multicolumn{2}{|c|}{ Average CPU } \\
\cline { 2 - 3 } \cline { 5 - 5 }$b$ & MIP No Cuts & DP Cuts & $b$ & MIP No Cuts & DP Cuts \\
\hline 1 & 16.6 & 17.5 & 12 & 34.8 & 54.5 \\
2 & 44.6 & 46.6 & 13 & 49.4 & 59.8 \\
3 & 70.3 & 69.4 & 14 & 28.0 & 31.2 \\
4 & 75.1 & 87.8 & 15 & 42.0 & 47.4 \\
5 & 84.8 & 92.3 & 16 & 43.4 & 57.6 \\
6 & 87.9 & 100.2 & 17 & 28.1 & 29.6 \\
7 & 59.9 & 53.7 & 18 & 16.5 & 44.0 \\
8 & 84.3 & 96.1 & 19 & 14.6 & 54.1 \\
9 & 100.1 & 109.6 & 20 & 14.8 & 60.5 \\
10 & 59.8 & 69.7 & 21 & 8.6 & 59.5 \\
11 & 68.4 & 83.0 & 22 & 4.4 & 56.0 \\
\hline
\end{tabular}

Table 5.11: Average CPU seconds for Inst_30 with DP inequalities.

\begin{tabular}{|c|c|c||c|c|c|}
\hline \multirow{2}{*}{$b$} & \multicolumn{2}{|c||}{ Average CPU } & & \multicolumn{2}{|c|}{ Average CPU } \\
\cline { 2 - 3 } & MIP No Cuts & DP Cuts & $b$ & MIP No Cuts & DP Cuts \\
\hline 1 & 29 & 30 & 15 & 227 & 291 \\
2 & 107 & 112 & 16 & 175 & 233 \\
3 & 207 & 212 & 17 & 146 & 193 \\
4 & 256 & 290 & 18 & 129 & 196 \\
5 & 265 & 272 & 19 & 90 & 94 \\
6 & 328 & 393 & 20 & 138 & 147 \\
7 & 380 & 412 & 21 & 114 & 109 \\
8 & 315 & 312 & 22 & 57 & 75 \\
9 & 176 & 235 & 23 & 51 & 168 \\
10 & 238 & 227 & 24 & 56 & 243 \\
11 & 272 & 343 & 25 & 12 & 230 \\
12 & 237 & 227 & 26 & 23 & 230 \\
13 & 172 & 245 & 27 & 12 & 206 \\
14 & 152 & 250 & & & \\
\hline
\end{tabular}


Table 5.12: Average CPU seconds for Inst_35 with DP inequalities.

\begin{tabular}{|c|c|c||c|c|c|}
\hline \multirow{3}{*}{$b$} & \multicolumn{2}{|c||}{ Average CPU } & & \multicolumn{2}{|c|}{ Average CPU } \\
\cline { 2 - 3 } \cline { 5 - 5 }$b$ & MIP No Cuts & DP Cuts & $b$ & MIP No Cuts & DP Cuts \\
\hline 1 & 81 & 81 & 17 & 1192 & 1108 \\
2 & 303 & 323 & 18 & 1062 & 1236 \\
3 & 597 & 627 & 19 & 1241 & 1084 \\
4 & 1014 & 1057 & 20 & 1154 & 886 \\
5 & 915 & 946 & 21 & 983 & 1094 \\
6 & 619 & 573 & 22 & 998 & 1032 \\
7 & 605 & 934 & 23 & 440 & 638 \\
8 & 1252 & 1228 & 24 & 546 & 607 \\
9 & 1116 & 1292 & 25 & 495 & 515 \\
10 & 1658 & 1790 & 26 & 352 & 487 \\
11 & 1334 & 1205 & 27 & 145 & 459 \\
12 & 1471 & 1103 & 28 & 224 & 1084 \\
13 & 1265 & 1364 & 29 & 98 & 814 \\
14 & 836 & 1011 & 30 & 60 & 723 \\
15 & 956 & 1086 & 31 & 70 & 709 \\
16 & 1293 & 1447 & 32 & 32 & 694 \\
\hline
\end{tabular}


Table 5.13: Average CPU seconds for Inst_40 with DP inequalities.

\begin{tabular}{|c|c|c||c|c|c|}
\hline \multirow{3}{*}{$b$} & \multicolumn{2}{|c||}{ Average CPU } & & \multicolumn{2}{|c|}{ Average CPU } \\
\cline { 2 - 3 }$b$ & MIP No Cuts & DP Cuts & $b$ & MIP No Cuts & DP Cuts \\
\hline 1 & 165 & 167 & 20 & 4995 & 4126 \\
2 & 367 & 301 & 21 & 5776 & 6093 \\
3 & 1094 & 1030 & 22 & 3148 & 2638 \\
4 & 1916 & 1956 & 23 & 2632 & 2912 \\
5 & 2604 & 2205 & 24 & 4008 & 3725 \\
6 & 1830 & 2589 & 25 & 2737 & 1373 \\
7 & 3048 & 3598 & 26 & 1371 & 1546 \\
8 & 3245 & 4258 & 27 & 1686 & 1928 \\
9 & 3237 & 3615 & 28 & 1145 & 1773 \\
10 & 2577 & 3198 & 29 & 521 & 936 \\
11 & 4822 & 5308 & 30 & 818 & 1307 \\
12 & 9044 & 7035 & 31 & 684 & 1914 \\
13 & 4322 & 5227 & 32 & 366 & 2767 \\
14 & 3282 & 3266 & 33 & 213 & 2375 \\
15 & 6616 & 6476 & 34 & 71 & 1972 \\
16 & 3540 & 6247 & 35 & 98 & 2056 \\
17 & 4128 & 5038 & 36 & 91 & 2186 \\
18 & 5479 & 5964 & 37 & 34 & 1769 \\
19 & 2172 & 3380 & & & \\
\hline
\end{tabular}


Table 5.14: Heuristic suboptimality for Inst_15

\begin{tabular}{|c|c|c|c|c|c|c|c|c|c|c|c|c|c|c|}
\hline \multirow{3}{*}{$b$} & \multicolumn{7}{|c|}{ Total Suboptimality } & \multicolumn{7}{|c|}{ Maximum Suboptimality } \\
\hline & \multirow[b]{2}{*}{ LP } & \multirow[b]{2}{*}{ DP1 } & \multirow[b]{2}{*}{ DP2 } & \multirow[b]{2}{*}{ DP3 } & \multicolumn{3}{|c|}{ MRGH } & \multirow[b]{2}{*}{ LP } & \multirow[b]{2}{*}{ DP1 } & \multirow[b]{2}{*}{ DP2 } & \multirow[b]{2}{*}{ DP3 } & \multicolumn{3}{|c|}{ MRGH } \\
\hline & & & & & 0 & $\overline{1}$ & 2 & & & & & 0 & 1 & 2 \\
\hline 0 & 0 & 0 & 0 & 0 & 1 & 0 & 0 & 0 & 0 & 0 & 0 & 1 & 0 & 0 \\
\hline 1 & 1 & 1 & 0 & 0 & 2 & 0 & 0 & 1 & 1 & 0 & 0 & 1 & 0 & 0 \\
\hline 2 & 2 & 1 & 0 & 0 & 3 & 1 & 1 & 1 & 1 & 0 & 0 & 1 & 1 & 1 \\
\hline 3 & 2 & 1 & 1 & 1 & 2 & 1 & 0 & 1 & 1 & 1 & 1 & 1 & 1 & 0 \\
\hline 4 & 2 & 1 & 1 & 0 & 2 & 0 & 0 & 1 & 1 & 1 & 0 & 1 & 0 & 0 \\
\hline 5 & 2 & 2 & 0 & 0 & 2 & 0 & 0 & 1 & 1 & 0 & 0 & 1 & 0 & 0 \\
\hline 6 & 3 & 2 & 1 & 1 & 2 & 0 & 0 & 1 & 1 & 1 & 1 & 1 & 0 & 0 \\
\hline 7 & 3 & 2 & 2 & 1 & 3 & 0 & 0 & 1 & 1 & 1 & 1 & 1 & 0 & 0 \\
\hline 8 & 1 & 0 & 0 & 0 & 0 & 0 & 0 & 1 & 0 & 0 & 0 & 0 & 0 & 0 \\
\hline 9 & 2 & 1 & 0 & 0 & 2 & 0 & 0 & 1 & 1 & 0 & 0 & 1 & 0 & 0 \\
\hline 10 & 1 & 1 & 1 & 0 & 0 & 0 & 0 & 1 & 1 & 1 & 0 & 0 & 0 & 0 \\
\hline 11 & 0 & 0 & 0 & 0 & 2 & 0 & 0 & 0 & 0 & 0 & 0 & 2 & 0 & 0 \\
\hline 12 & 3 & 0 & 0 & 0 & 3 & 0 & 0 & 1 & 0 & 0 & 0 & 1 & 0 & 0 \\
\hline
\end{tabular}


Table 5.15: Heuristic suboptimality for Inst_20

\begin{tabular}{|c|c|c|c|c|c|c|c|c|c|c|c|c|c|c|}
\hline \multirow{3}{*}{$b$} & \multicolumn{7}{|c|}{ Total Suboptimality } & \multicolumn{7}{|c|}{ Maximum Suboptimality } \\
\hline & \multirow[b]{2}{*}{ LP } & \multirow[b]{2}{*}{ DP1 } & \multirow[b]{2}{*}{ DP2 } & \multirow[b]{2}{*}{ DP3 } & \multicolumn{3}{|c|}{ MRGH } & \multirow[b]{2}{*}{ LP } & \multirow[b]{2}{*}{ DP1 } & \multirow[b]{2}{*}{ DP2 } & \multirow[b]{2}{*}{ DP3 } & \multicolumn{3}{|c|}{ MRGH } \\
\hline & & & & & 0 & 1 & 2 & & & & & 0 & 1 & 2 \\
\hline 0 & 0 & 0 & 0 & 0 & 1 & 0 & 0 & 0 & 0 & 0 & 0 & 1 & 0 & 0 \\
\hline 1 & 2 & 6 & 1 & 1 & 3 & 2 & 1 & 1 & 2 & 1 & 1 & 2 & 1 & 1 \\
\hline 2 & 6 & 3 & 1 & 0 & 3 & 0 & 0 & 2 & 1 & 1 & 0 & 1 & 0 & 0 \\
\hline 3 & 2 & 5 & 2 & 2 & 5 & 0 & 0 & 1 & 2 & 1 & 1 & 2 & 0 & 0 \\
\hline 4 & 1 & 2 & 1 & 0 & 4 & 0 & 0 & 1 & 1 & 1 & 0 & 2 & 0 & 0 \\
\hline 5 & 1 & 0 & 0 & 0 & 1 & 0 & 0 & 1 & 0 & 0 & 0 & 1 & 0 & 0 \\
\hline 6 & 0 & 3 & 1 & 0 & 4 & 0 & 0 & 0 & 1 & 1 & 0 & 1 & 0 & 0 \\
\hline 7 & 1 & 2 & 0 & 0 & 1 & 0 & 0 & 1 & 1 & 0 & 0 & 1 & 0 & 0 \\
\hline 8 & 2 & 4 & 2 & 1 & 6 & 0 & 0 & 1 & 1 & 1 & 1 & 2 & 0 & 0 \\
\hline 9 & 3 & 2 & 0 & 0 & 2 & 0 & 0 & 1 & 1 & 0 & 0 & 1 & 0 & 0 \\
\hline 10 & 1 & 3 & 1 & 0 & 5 & 1 & 0 & 1 & 1 & 1 & 0 & 3 & 1 & 0 \\
\hline 11 & 1 & 3 & 0 & 0 & 5 & 2 & 0 & 1 & 1 & 0 & 0 & 2 & 2 & 0 \\
\hline 12 & 1 & 2 & 0 & 0 & 5 & 0 & 0 & 1 & 1 & 0 & 0 & 1 & 0 & 0 \\
\hline 13 & 0 & 1 & 0 & 0 & 2 & 0 & 0 & 0 & 1 & 0 & 0 & 1 & 0 & 0 \\
\hline 14 & 3 & 1 & 1 & 1 & 3 & 2 & 0 & 1 & 1 & 1 & 1 & 1 & 1 & 0 \\
\hline 15 & 3 & 0 & 0 & 0 & 1 & 0 & 0 & 1 & 0 & 0 & 0 & 1 & 0 & 0 \\
\hline 16 & 0 & 0 & 0 & 0 & 1 & 0 & 0 & 0 & 0 & 0 & 0 & 1 & 0 & 0 \\
\hline 17 & 3 & 0 & 0 & 0 & 3 & 0 & 0 & 1 & 0 & 0 & 0 & 1 & 0 & 0 \\
\hline
\end{tabular}


Table 5.16: Heuristic suboptimality for Inst_25

\begin{tabular}{|c|c|c|c|c|c|c|c|c|c|c|c|c|c|c|}
\hline \multirow{3}{*}{$b$} & \multicolumn{7}{|c|}{ Total Suboptimality } & \multicolumn{7}{|c|}{ Maximum Suboptimality } \\
\hline & \multirow[b]{2}{*}{ LP } & \multirow[b]{2}{*}{ DP1 } & \multirow[b]{2}{*}{ DP2 } & \multirow[b]{2}{*}{ DP3 } & \multicolumn{3}{|c|}{ MRGH } & \multirow[b]{2}{*}{ LP } & \multirow[b]{2}{*}{ DP1 } & \multirow[b]{2}{*}{ DP2 } & \multirow[b]{2}{*}{ DP3 } & \multicolumn{3}{|c|}{ MRGH } \\
\hline & & & & & 0 & 1 & 2 & & & & & 0 & 1 & 2 \\
\hline 0 & 0 & 0 & 0 & 0 & 6 & 2 & 0 & 0 & 0 & 0 & 0 & 1 & 1 & 0 \\
\hline 1 & 8 & 9 & 5 & 4 & 6 & 2 & 1 & 2 & 2 & 2 & 1 & 3 & 1 & 1 \\
\hline 2 & 4 & 14 & 6 & 2 & 10 & 3 & 1 & 1 & 3 & 1 & 1 & 2 & 1 & 1 \\
\hline 3 & 8 & 8 & 7 & 4 & 10 & 3 & 1 & 2 & 1 & 1 & 1 & 2 & 1 & 1 \\
\hline 4 & 2 & 8 & 4 & 0 & 9 & 2 & 1 & 1 & 1 & 1 & 0 & 2 & 2 & 1 \\
\hline 5 & 5 & 11 & 4 & 1 & 8 & 3 & 1 & 2 & 2 & 1 & 1 & 1 & 1 & 1 \\
\hline 6 & 4 & 8 & 0 & 0 & 13 & 1 & 0 & 1 & 2 & 0 & 0 & 4 & 1 & 0 \\
\hline 7 & 7 & 10 & 4 & 0 & 8 & 2 & 0 & 2 & 2 & 2 & 0 & 2 & 1 & 0 \\
\hline 8 & 4 & 7 & 4 & 0 & 7 & 1 & 0 & 1 & 2 & 1 & 0 & 2 & 1 & 0 \\
\hline 9 & 3 & 2 & 2 & 0 & 4 & 1 & 0 & 1 & 1 & 1 & 0 & 1 & 1 & 0 \\
\hline 10 & 4 & 5 & 3 & 0 & 6 & 0 & 0 & 1 & 1 & 1 & 0 & 1 & 0 & 0 \\
\hline 11 & 5 & 5 & 2 & 1 & 5 & 0 & 1 & 2 & 2 & 1 & 1 & 1 & 0 & 1 \\
\hline 12 & 3 & 5 & 4 & 0 & 8 & 1 & 0 & 1 & 1 & 1 & 0 & 2 & 1 & 0 \\
\hline 13 & 4 & 3 & 1 & 1 & 7 & 1 & 1 & 2 & 1 & 1 & 1 & 2 & 1 & 1 \\
\hline 14 & 4 & 6 & 1 & 1 & 4 & 2 & 0 & 1 & 2 & 1 & 1 & 1 & 1 & 0 \\
\hline 15 & 1 & 2 & 1 & 0 & 9 & 0 & 0 & 1 & 1 & 1 & 0 & 3 & 0 & 0 \\
\hline 16 & 3 & 2 & 0 & 0 & 8 & 0 & 0 & 1 & 1 & 0 & 0 & 3 & 0 & 0 \\
\hline 17 & 4 & 3 & 1 & 1 & 7 & 0 & 0 & 1 & 1 & 1 & 1 & 3 & 0 & 0 \\
\hline 18 & 2 & 2 & 1 & 0 & 5 & 0 & 0 & 1 & 1 & 1 & 0 & 2 & 0 & 0 \\
\hline 19 & 3 & 3 & 0 & 0 & 5 & 0 & 0 & 1 & 1 & 0 & 0 & 2 & 0 & 0 \\
\hline 20 & 2 & 0 & 0 & 0 & 2 & 0 & 0 & 1 & 0 & 0 & 0 & 1 & 0 & 0 \\
\hline 21 & 1 & 0 & 0 & 0 & 0 & 0 & 0 & 1 & 0 & 0 & 0 & 0 & 0 & 0 \\
\hline 22 & 5 & 0 & 0 & 0 & 3 & 0 & 0 & 1 & 0 & 0 & 0 & 1 & 0 & 0 \\
\hline
\end{tabular}


Table 5.17: Heuristic suboptimality for Inst_30

\begin{tabular}{|c|c|c|c|c|c|c|c|c|c|c|c|c|c|c|}
\hline \multirow{3}{*}{$b$} & \multicolumn{7}{|c|}{ Total Suboptimality } & \multicolumn{7}{|c|}{ Maximum Suboptimality } \\
\hline & \multirow[b]{2}{*}{ LP } & \multirow[b]{2}{*}{ DP1 } & \multirow[b]{2}{*}{ DP2 } & \multirow[b]{2}{*}{ DP3 } & \multicolumn{3}{|c|}{ MRGH } & \multirow[b]{2}{*}{ LP } & \multirow[b]{2}{*}{ DP1 } & \multirow[b]{2}{*}{ DP2 } & \multirow[b]{2}{*}{ DP3 } & \multicolumn{3}{|c|}{ MRGH } \\
\hline & & & & & 0 & 1 & 2 & & & & & 0 & 1 & 2 \\
\hline 0 & 0 & 0 & 0 & 0 & 6 & 1 & 0 & 0 & 0 & 0 & 0 & 1 & 1 & 0 \\
\hline 2 & 2 & 9 & 3 & 0 & 8 & 3 & 1 & 1 & 3 & 2 & 0 & 2 & 1 & 1 \\
\hline 4 & 6 & 11 & 4 & 3 & 10 & 3 & 3 & 2 & 2 & 1 & 1 & 2 & 1 & 1 \\
\hline 6 & 6 & 7 & 5 & 2 & 6 & 2 & 1 & 1 & 2 & 1 & 1 & 1 & 1 & 1 \\
\hline 8 & 6 & 4 & 3 & 3 & 4 & 2 & 0 & 2 & 2 & 2 & 2 & 2 & 1 & 0 \\
\hline 10 & 8 & 4 & 1 & 1 & 4 & 2 & 0 & 2 & 1 & 1 & 1 & 1 & 1 & 0 \\
\hline 12 & 2 & 3 & 2 & 0 & 6 & 0 & 0 & 1 & 1 & 1 & 0 & 2 & 0 & 0 \\
\hline 14 & 2 & 3 & 1 & 0 & 7 & 0 & 0 & 1 & 1 & 1 & 0 & 2 & 0 & 0 \\
\hline 16 & 6 & 2 & 2 & 0 & 7 & 1 & 0 & 1 & 1 & 1 & 0 & 1 & 1 & 0 \\
\hline 18 & 3 & 3 & 1 & 0 & 7 & 3 & 0 & 1 & 1 & 1 & 0 & 4 & 2 & 0 \\
\hline 20 & 1 & 2 & 0 & 0 & 5 & 0 & 0 & 1 & 1 & 0 & 0 & 4 & 0 & 0 \\
\hline 22 & 4 & 1 & 0 & 0 & 3 & 0 & 0 & 1 & 1 & 0 & 0 & 1 & 0 & 0 \\
\hline 24 & 3 & 2 & 2 & 2 & 4 & 1 & 0 & 1 & 1 & 1 & 1 & 1 & 1 & 0 \\
\hline 26 & 2 & 0 & 0 & 0 & 2 & 0 & 0 & 1 & 0 & 0 & 0 & 1 & 0 & 0 \\
\hline
\end{tabular}


Table 5.18: Heuristic suboptimality for Inst_35

\begin{tabular}{|c|c|c|c|c|c|c|c|c|c|c|c|c|c|c|}
\hline \multirow{3}{*}{$b$} & \multicolumn{7}{|c|}{ Total Suboptimality } & \multicolumn{7}{|c|}{ Maximum Suboptimality } \\
\hline & \multirow[b]{2}{*}{ LP } & \multirow[b]{2}{*}{ DP1 } & \multirow[b]{2}{*}{ DP2 } & \multirow[b]{2}{*}{ DP3 } & \multicolumn{3}{|c|}{ MRGH } & \multirow[b]{2}{*}{ LP } & \multirow[b]{2}{*}{ DP1 } & \multirow[b]{2}{*}{ DP2 } & \multirow[b]{2}{*}{ DP3 } & \multicolumn{3}{|c|}{ MRGH } \\
\hline & & & & & 0 & 1 & 2 & & & & & 0 & 1 & 2 \\
\hline 0 & 0 & 0 & 0 & 0 & 11 & 1 & 0 & 0 & 0 & 0 & 0 & 3 & $\overline{1}$ & 0 \\
\hline 2 & 6 & 15 & 8 & 1 & 8 & 2 & 1 & 1 & 3 & 2 & 1 & 2 & 1 & 1 \\
\hline 4 & 6 & 11 & 6 & 0 & 9 & 2 & 0 & 1 & 2 & 1 & 0 & 2 & 1 & 0 \\
\hline 6 & 5 & 14 & 3 & 1 & 9 & 2 & 1 & 2 & 4 & 1 & 1 & 2 & 1 & 1 \\
\hline 8 & 3 & 8 & 4 & 3 & 9 & 2 & 0 & 1 & 2 & 2 & 1 & 2 & 1 & 0 \\
\hline 10 & 2 & 6 & 2 & 1 & 7 & 1 & 0 & 1 & 2 & 1 & 1 & 1 & 1 & 0 \\
\hline 12 & 6 & 11 & 5 & 1 & 9 & 5 & 2 & 1 & 2 & 1 & 1 & 2 & 2 & 1 \\
\hline 14 & 3 & 11 & 2 & 2 & 6 & 1 & 0 & 1 & 2 & 1 & 1 & 1 & 1 & 0 \\
\hline 16 & 2 & 10 & 1 & 1 & 6 & 2 & 0 & 1 & 2 & 1 & 1 & 1 & 1 & 0 \\
\hline 18 & 4 & 10 & 1 & 1 & 9 & 4 & 0 & 1 & 2 & 1 & 1 & 3 & 2 & 0 \\
\hline 20 & 4 & 7 & 1 & 1 & 9 & 3 & 0 & 2 & 2 & 1 & 1 & 2 & 1 & 0 \\
\hline 22 & 4 & 6 & 2 & 1 & 10 & 2 & 1 & 1 & 1 & 1 & 1 & 5 & 1 & 1 \\
\hline 24 & 1 & 2 & 0 & 0 & 9 & 0 & 0 & 1 & 1 & 0 & 0 & 5 & 0 & 0 \\
\hline 26 & 5 & 2 & 2 & 0 & 9 & 1 & 0 & 1 & 1 & 1 & 0 & 2 & 1 & 0 \\
\hline 28 & 3 & 2 & 0 & 0 & 1 & 0 & 0 & 1 & 1 & 0 & 0 & 1 & 0 & 0 \\
\hline 30 & 2 & 1 & 0 & 0 & 5 & 1 & 0 & 1 & 1 & 0 & 0 & 1 & 1 & 0 \\
\hline 32 & 6 & 0 & 0 & 0 & 5 & 0 & 1 & 1 & 0 & 0 & 0 & 1 & 0 & 1 \\
\hline
\end{tabular}


Table 5.19: Heuristic suboptimality for Inst_40

\begin{tabular}{|c|c|c|c|c|c|c|c|c|c|c|c|c|c|c|}
\hline \multirow{3}{*}{$b$} & \multicolumn{7}{|c|}{ Total Suboptimality } & \multicolumn{7}{|c|}{ Maximum Suboptimality } \\
\hline & \multirow[b]{2}{*}{ LP } & \multirow[b]{2}{*}{ DP1 } & \multirow[b]{2}{*}{ DP2 } & \multirow[b]{2}{*}{ DP3 } & \multicolumn{3}{|c|}{$\mathrm{MRGH}$} & \multirow[b]{2}{*}{ LP } & \multirow[b]{2}{*}{ DP1 } & \multirow[b]{2}{*}{ DP2 } & \multirow[b]{2}{*}{ DP3 } & \multicolumn{3}{|c|}{ MRGH } \\
\hline & & & & & 0 & 1 & 2 & & & & & 0 & 1 & 2 \\
\hline 0 & 0 & 0 & 0 & 0 & 3 & 0 & 0 & 0 & 0 & 0 & 0 & 2 & 0 & 0 \\
\hline 2 & 2 & 11 & 6 & 0 & 11 & 1 & 0 & 1 & 2 & 2 & 0 & 2 & 1 & 0 \\
\hline 4 & 3 & 13 & 7 & 2 & 12 & 3 & 0 & 1 & 3 & 2 & 1 & 2 & 1 & 0 \\
\hline 6 & 8 & 17 & 10 & 1 & 11 & 4 & 0 & 1 & 2 & 2 & 1 & 2 & 1 & 0 \\
\hline 8 & 7 & 15 & 7 & 1 & 12 & 4 & 0 & 2 & 2 & 2 & 1 & 2 & 1 & 0 \\
\hline 10 & 8 & 12 & 4 & 3 & 11 & 5 & 1 & 2 & 3 & 2 & 1 & 2 & 1 & 1 \\
\hline 12 & 4 & 7 & 2 & 2 & 10 & 4 & 0 & 2 & 2 & 1 & 1 & 2 & 1 & 0 \\
\hline 14 & 7 & 8 & 6 & 4 & 10 & 3 & 1 & 1 & 2 & 1 & 1 & 2 & 1 & 1 \\
\hline 16 & 4 & 5 & 5 & 3 & 7 & 1 & 0 & 1 & 1 & 1 & 1 & 2 & 1 & 0 \\
\hline 18 & 4 & 8 & 3 & 3 & 6 & 2 & 0 & 1 & 2 & 1 & 1 & 1 & 1 & 0 \\
\hline 20 & 5 & 7 & 2 & 1 & 8 & 1 & 0 & 1 & 1 & 1 & 1 & 2 & 1 & 0 \\
\hline 22 & 2 & 6 & 2 & 0 & 4 & 2 & 0 & 1 & 2 & 1 & 0 & 1 & 1 & 0 \\
\hline 24 & 1 & 3 & 1 & 0 & 6 & 1 & 0 & 1 & 1 & 1 & 0 & 1 & 1 & 0 \\
\hline 26 & 3 & 5 & 0 & 0 & 6 & 0 & 0 & 2 & 1 & 0 & 0 & 2 & 0 & 0 \\
\hline 28 & 5 & 6 & 2 & 0 & 4 & 2 & 0 & 1 & 2 & 1 & 0 & 1 & 1 & 0 \\
\hline 30 & 2 & 1 & 0 & 0 & 4 & 1 & 0 & 1 & 1 & 0 & 0 & 1 & 1 & 0 \\
\hline 32 & 3 & 3 & 0 & 0 & 7 & 0 & 0 & 1 & 1 & 0 & 0 & 4 & 0 & 0 \\
\hline 34 & 3 & 3 & 1 & 1 & 6 & 1 & 0 & 1 & 1 & 1 & 1 & 1 & 1 & 0 \\
\hline 36 & 2 & 1 & 0 & 0 & 3 & 0 & 0 & 1 & 1 & 0 & 0 & 1 & 0 & 0 \\
\hline
\end{tabular}

Table 5.20: Heuristic average CPU times in seconds.

\begin{tabular}{|c|c|c|c|c|c|c|c|c|}
\hline & \multicolumn{9}{|c|}{ Average CPU Times } \\
\cline { 6 - 9 } Inst. & & & & & & \multicolumn{3}{|c|}{ MRGH } \\
\cline { 6 - 9 } Set & MIP & LP & DP1 & DP2 & DP3 & 0 & 1 & 2 \\
\hline Inst_15 & 0.75 & 0.70 & 0.06 & 0.19 & 0.56 & 0.03 & 0.18 & 1.07 \\
Inst_20 & 4.10 & 3.21 & 0.16 & 0.71 & 2.32 & 0.07 & 0.92 & 7.84 \\
Inst_25 & 45.10 & 15.50 & 0.30 & 1.65 & 6.05 & 0.18 & 3.54 & 41.73 \\
Inst_30 & 155.96 & 49.10 & 0.56 & 3.97 & 15.19 & 0.39 & 10.06 & 147.21 \\
Inst_35 & 739.72 & 167.64 & 1.04 & 9.91 & 51.47 & 0.81 & 23.99 & 432.53 \\
Inst_40 & 2470.65 & 438.71 & 1.69 & 19.36 & 126.64 & 1.55 & 62.18 & 1220.43 \\
\hline
\end{tabular}




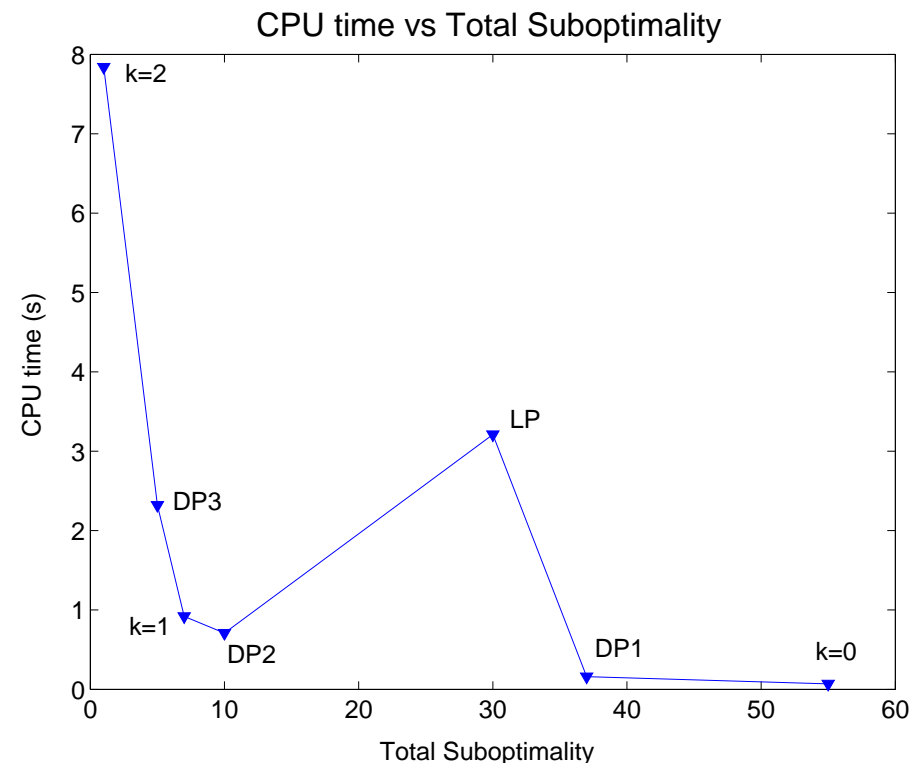

Figure 5.5: CPU seconds versus total suboptimality for the instance set Inst_20.

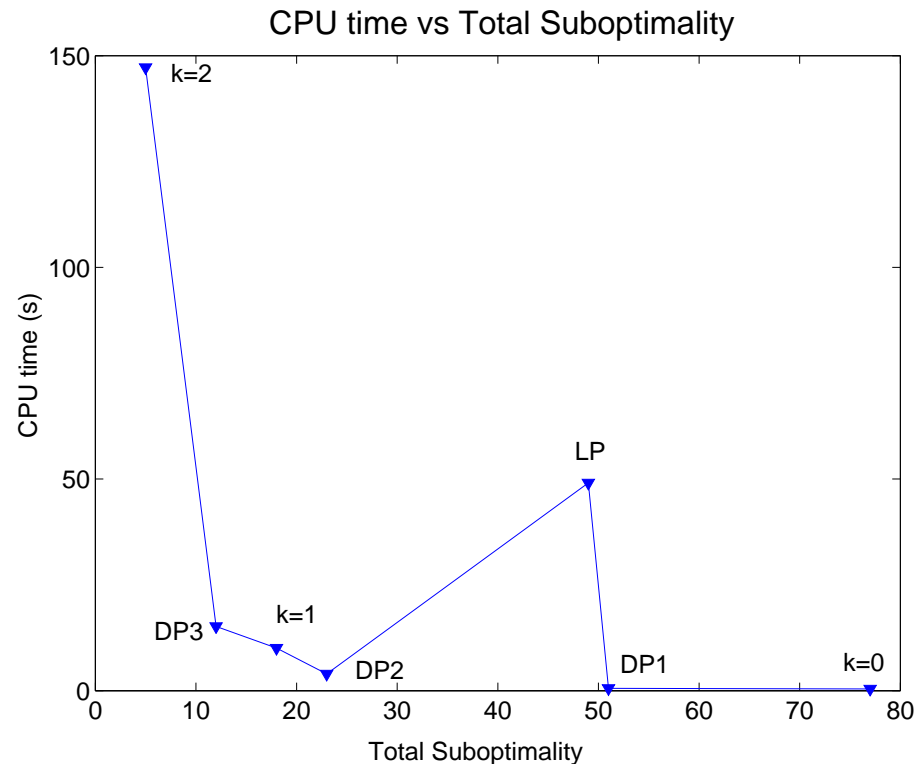

Figure 5.6: CPU seconds versus total suboptimality for the instance set Inst_30. 


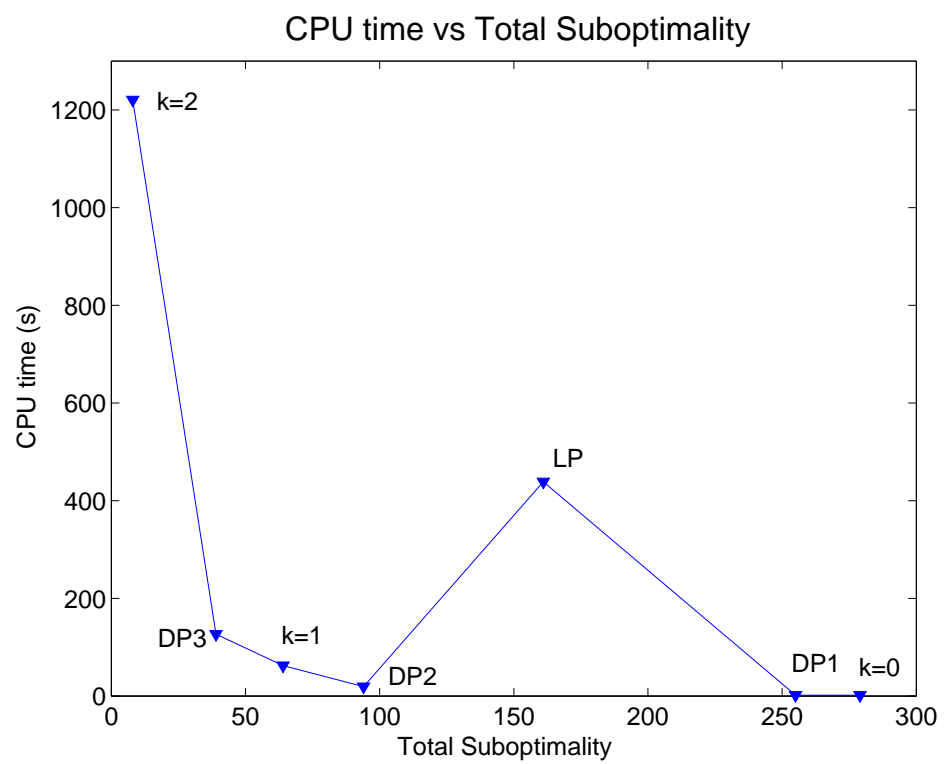

Figure 5.7: CPU seconds versus total suboptimality for the instance set Inst_40. 


\subsection{Summary}

In this chapter, we derived valid inequalities for the GMFPP by identifying polynomially solvable independent subgraphs of the underlying communication network. We showed that the inequalities are facet-inducing to the respective independent subgraphs. In addition, we derived valid inequalities based the dynamic programming algorithm for solving the GMFPP on tree networks. We recommend the use of star inequalities even when the underlying network has no special structure. Also, when the network contains a large chain subgraph, we recommend the inclusion of a corresponding chain inequality. Finally, for networks involving a large clique subgraph, we recommend the inclusion of a clique inequality, particularly when $b$ is small relative to $n$.

We also provided three heuristics for solving the GMFPP. Computational results showed that the multiple restart greedy heuristic with $k=2$ outperformed the other heuristics in terms of solution quality, but it was computationally expensive. While the dynamic programming-based inequalities did not improve the solvability of the mixed-integer program, the dynamic programming-based heuristic with postprocessing yielded better results than both the linear programming-based heuristic and the multiple restart greed heuristic with $k=1$ at a relatively low computational cost. 


\section{CHAPTER 6}

\section{Conclusions and Future Work}

We conclude with a summary of our work in Section 6.1 and state directions for future research motivated by the work presented in this dissertation in Section 6.2.

\subsection{Conclusions and Summary}

We presented the Generalized Minimum Filter Placement Problem and formulated it as a mixed-integer program. We identified special network topologies for which the GMFPP is solvable in polynomial time in Chapter 4, and presented a polynomialtime dynamic programming algorithm for solving the GMFPP on tree networks. The algorithm works by examining subtrees starting from leaf nodes, and progressing systematically towards the root node. We showed that the computational complexity of the dynamic program is $O\left(n^{5}\right)$. Computational results showed that the algorithm can solve much larger problem instances within reasonable computational limits than can a mixed-integer programming technique.

In Chapter 5, we derived valid inequalities for the GMFPP by identifying polynomially solvable independent subgraphs of the underlying communication network. We showed that the inequalities are facet-inducing to the respective independent subgraphs. We also derived valid inequalities based on the dynamic programming algorithm for solving the GMFPP on tree networks, and we provided three heuristics for solving the GMFPP. Computational results showed that the multiple restart greedy heuristic with $k=2$ outperformed the other heuristic in terms of solution quality but it was computationally expensive. The dynamic programmingbased heuristic with post-processing yielded better results than both the linear programming-based heuristic and the multiple restart greed heuristic with $k=1$ at a relatively low computational cost. 


\subsection{Future Work}

Future tasks motivated by the study presented in this dissertation include seeking to develop a polynomial-time constant-factor approximation algorithm for the GMFPP on general graphs based on one of the heuristic algorithms we presented. Another interesting avenue is to investigate the applicability of our findings to other similar practical problems. (For instance, identifying the best locations to set up checkpoints for the U.S. Border Patrol.)

Often, there are different overheads associated with performing filtering operations at different nodes. As a future research direction, we propose to explore a variant of the GMFPP in which we associate a positive weight with each node of the network. These weights are set proportional to the overhead that we may incur by filtering the corresponding nodes. The objective of the GMFPP would then be to minimize the weighted number of filters to deploy in the network so that the desired level of security is attained.

Another future research direction is to investigate the applicability of ideas and results from this study to the verification of workflow models. The Workflow Management Coalition (WfMC) defines workflow as the automation of a business process, in whole or part, during which documents, information or tasks are passed from one participant to another for action, according to a set of procedural rules [79]. A work-

flow model is usually represented by a directed graph (called the workflow graph) containing two types of nodes: activity nodes and routing nodes. The arc set represents interdependencies and information flow amongst activities. Before defining the problem of identifying structural conflicts in workflow models, we give a cursory introduction to the graphical representation of workflow models, adopting the notation in [7].

Figure 6.1 shows the basic components used to construct graphical representations of workflow models. The Start vertex and the End vertex shown in Figure 6.1a are special activity vertices representing the starting point and ending point of a workflow, respectively. An AND vertex is said to be an AND-split if it has 


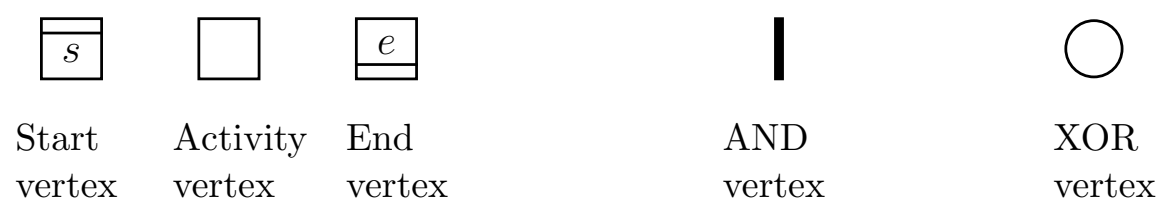
(a) Activity vertices
(b) Routing vertices

Figure 6.1: Basic constructs of workflow models.



AND-split



XOR-split

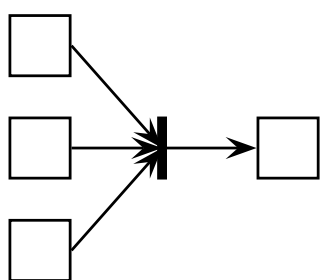

AND-join

(a)

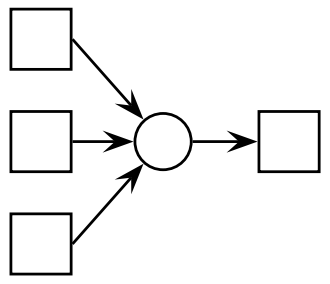

XOR-join

(b)

Figure 6.2: Split and join routing structures.

one incoming arc and multiple outgoing arcs, and an AND-join if it has multiple incoming arcs and a single outgoing arc (Figure 6.2a). XOR-split and XOR-join are define in a similar manner as shown in Figure 6.2b.

An arc is said to be activated if the node incident to its tail has just completed executing. A vertex may start executing only if its incoming arc is active, and once 


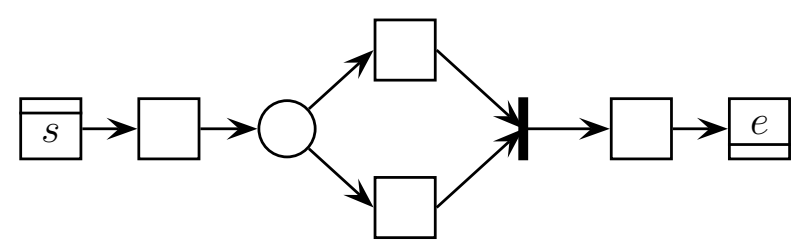

Figure 6.3: An example of a deadlock.

execution starts, the incoming arc is deactivated. If an incoming arc to an ANDsplit vertex is activated, the AND vertex activates all outgoing arc (at the same time deactivating the incoming arc). An AND-join vertex will activate the outgoing arc if and only if all incoming arcs have been activated. When an incoming arc to the XOR-split vertex is activated, exactly one of the outgoing arcs is activated. The outgoing arc from an XOR-join vertex is activated when exactly one of the in coming arcs is active. Having more than one incoming arcs of the XOR-join vertex active leads to an undesirable multiple activation of the outgoing arc.

The rules governing the activation of the outgoing arc of the AND-join and XORjoin vertices may lead to two structural conflicts in a workflow graphs: deadlock and lack of synchronization. A deadlock occurs when only a proper subset of incoming arcs of an AND-join vertex are activated; therefore, the outgoing arc in never activated. An example of a workflow graph with a deadlock is shown in Figure 6.3. In this simple illustration, the XOR-split vertex will activate exactly one outgoing arc, implying that the AND-join vertex will only have one of the two incoming arcs active; hence, the outgoing arc of the AND-join vertex will never be activated. A lack of synchronization occurs when more than one incoming arcs of an XOR-join vertex are activated, resulting into unintentional multiple activation of the outgoing arc. Figure 6.4 shows an example workflow graph with a lack of synchronization conflict.

The problem we consider for future research seeks to identify any deadlock and/or lack of synchronization conflicts that may be present in a given workflow graph (or 


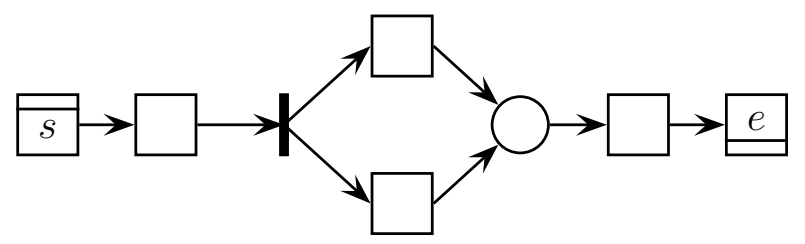

Figure 6.4: An example of a lack of synchronization.

to validate that the workflow graph is free from such conflicts). While the GMFPP is involves validating the authenticity of communication packets, this problem assumes that the information flowing in the network is legitimate, and is concerned with validating the correctness of the network itself.

In general, the problem of determining whether or not a workflow graph contains structural conflicts is NP-hard [37]. Therefore, we can justifiably model the problem as an integer program. We can apply ideas presented in the dissertation to increase the solvability of the integer programming model. For instances, we could focus our attention on finding special cases of of workflow graphs that can be solved in polynomial time; we can then use the insights obtained from these cases to generate cutting planes for the integer programming model of a general workflow graph. 


\section{REFERENCES}

[1] Adler, M. (2002). Tradeoffs in Probabilistic Packet Marking for IP Traceback. In STOC '02: Proceedings of the thiry-fourth annual ACM symposium on Theory of computing, pp. 407-418. Montreal, Quebec, Canada.

[2] Al-Duwairi, B. and G. Manimaran (2004). A Novel Packet Marking Scheme for IP Traceback. In Tenth International Conference on Parallel and Distributed Systems (ICPADS '04), pp. 195-202.

[3] Aljifri, H. (2003). IP Traceback: A New Denial-of-Service Deterrent? IEEE Security \& Privacy Magazine, 1(3), pp. 24-31.

[4] Armbruster, B., J. C. Smith, and K. Park (2005). A Packet Filter Placement Problem with Application to Defense Against Distributed Denial of Service Attacks. Working Paper, The University of Arizona, Tucson, AZ.

[5] Aura, T., P. Nikander, and J. Leiwo (2001). DoS-Resistant Authentication with Client Puzzles. In Revised Papers from the 8th International Workshop on Security Protocols, pp. 170-177. Springer-Verlag, London, UK.

[6] Belenky, A. and N. Ansari (2003). IP Traceback with Deterministic Packet Marking. IEEE Communications Letters, 7(4), pp. 162-164.

[7] Bi, H. H. and J. L. Zhao (2004). Process Logic For Verifying the Correctness of Business Process Models. In International Conference on Information Systems (ICIS 2004), pp. 91-100. Washington, D.C.

[8] Blazek, R. B., H. Kim, B. Rozovskii, and A. Tartakovsky (2001). A Novel Approach to Detection of Denial-of-Service Attacks via Adaptive Sequential and Batch-Sequential Change-Point Tetection Methods. In IEEE Systems, Man, and Cybernetics Information Assurance Workshop. West Point, NY. 
[9] Brustoloni, J. C. (2002). Protecting Electronic Commerce from Distributed Denial-of-Service Attacks. In $W W W$ '02: Proceedings of the 11th international conference on World Wide Web, pp. 553-561. Honolulu, Hawaii, USA.

[10] Burch, H. and B. Cheswick (2000). Tracing Anonymous Packets to Their Approximate Source. In LISA, pp. 319-327. New Orleans, LA.

[11] CERT (2000). Advisory CA-2000-01 Denial-of-Service Developments. http: //www.cert.org/advisories/CA-2000-01.html. Computer Emergency Response Team (CERT).

[12] CERT (2000). Advisory CA-2000-01 Denial-of-service developments. http: //www.cert.org/advisories/CA-2000-01.html. Computer Emergency Response Team (CERT).

[13] Chang, R. K. C. (2002). Defending Against Flooding-Based Distributed Denialof-Service Attacks: A Tutorial. IEEE Communications Magazine, 40(10), pp. $42-51$.

[14] Chen, L., T. A. Longstaff, and K. M. Carley (2004). Characterization of defense mechanisms against distributed denial of service attacks. Computers $\&$ Security, 23, pp. 665-678.

[15] Chen, Y., A. Bargteil, D. Bindel, R. H. Katz, and J. Kubiatowicz (2001). Quantifying Network Denial of Service: A Location Service Case Study. In ICICS '01: Proceedings of the Third International Conference on Information and Communications Security, pp. 340-351. Springer-Verlag, London, UK.

[16] Cherazi, G. and S. Koch (2005). Denial of Service Attacks in IP Networks. Technical report, Information Security, Linköping Universitet, Linköping, Sweden.

[17] Choi, Y. and J. L. Zhao (2002). Matrix-Based Abstraction and Verification for e-Business Processes. In Proceedings of the 1st Workshop on e-Business, pp. 154-165. Barcelona, Spain. 
[18] Choi, Y. and J. L. Zhao (2003). Handling Cycles in Workflow Verification by Feedback Identification and Partition. In Proceedings of the 2003 International Conference on Information and Knowledge Engineering, pp. 337-343. Las Vegas, Nevada.

[19] Cisco Systems, Inc. (1999). Defining Strategies to Protect Against TCP SYN Denial of Service Attacks. Available at http://cio.cisco.com/warp/public/ 707/4.html.

[20] Crosby, S. and D. Wallach (2003). Denial of Service via Algorithmic Complexity Attacks. In Proceedings of the 12th USENIX Security Symposium, pp. 29-44. Washington, DC.

[21] CSI/FBI (2002). Computer Crime and Security Survey. Computer Security Institute and Federal Bureau of Investigation. Available from http://www. gocsi.com.

[22] CSI/FBI (2003). Computer Crime and Security Survey. Computer Security Institute and Federal Bureau of Investigation. Available from http://www. gocsi.com.

[23] CSI/FBI (2004). Computer Crime and Security Survey. Computer Security Institute and Federal Bureau of Investigation. Available from http://www. gocsi.com.

[24] Dean, D., M. Franklin, and A. Stubblefield (2002). An Algebraic Approach to IP Traceback. ACM Transactions on Information and System Security, 5(2), pp. 119-137.

[25] Dietrich, S., N. Long, and D. Dittrich (2000). Analyzing Distributed Denial of Service Tools: The Shaft Case. In 14th System Administration Conference (LISA 2000), pp. 329-339. New Orleans, Louisiana.

[26] Douligeris, C. and A. Mitrokotsa (2004). DDoS attacks and defense mechanisms: classification and state-of-the-art. Computer Networks, 44, pp. 643-666. 
[27] Dübendorfer, T., M. Bossardt, and B. Plattner (2005). Adaptive Distributed Traffic Control Service for DDoS Attack Mitigation. In 19th IEEE International Parallel and Distributed Processing Symposium, (IPDPS'05). Denver, CO.

[28] Farraposo, S., L. Gallon, and P. Owezarski (2005). Network Security and DoS Attacks. Technical report, LAAS-CNRS, France.

[29] Ferguson, P. and D. Senie (2000). Network ingress filtering: Defeating denial of service attacks which employ IP source address spoofing. RCF 2827.

[30] Garber, L. (2000). Denial-of-Service Attacks Rip the Internet. IEEE Computer, 33(4), pp. 12-17.

[31] Garey, M. R. and D. S. Johnson (1979). Computers and Intractability: A Guide to the Theory of NP-Completeness. W.H. Freeman and Co., New York, NY.

[32] Garg, A. and N. A. L. Reddy (2002). Mitigating Denial of Service Using QoS Regulation. In International Workshop on Quality of Service. Miami Beach, FL.

[33] Geng, X., Y. Huang, and A. B. Whinston (2002). Defending Wireless Infrastructure Against the Challenge of DDoS Attacks. Mobile Network and Applications, $7(3)$, pp. 213-223.

[34] Gregg, D. M., W. J. Blackert, D. V. Heinbuch, and D. C. Furnanage (2001). Analyzing Denial of Service Attacks Using Theory and Modeling and Simulation. In IEEE Workshop on Information Assurance and Security, pp. 205-211.

[35] Habib, A., M. M. Hefeeda, and B. K. Bhargava (2003). Detecting Service Violations and DoS Attacks. In Network and Distributed System Security Symposium (NDSS '03), pp. 117-189. San Diego, CA.

[36] Hawkinson, J. and T. Bates (1996). Guidelines for Creation, Selection, and Registration of an Autonomous System AS. Available at http://www. ietf . org/rfc/rfc1930.txt. RFC 1930. 
[37] Hofstede, A., M. Orlowska, and J. Rajapakse (1996). Verification Problems in Conceptual Workflow Specifications. Technical report, Queensland University of Technology, GPO Box 2434, Brisbane Qld 4001, Australia.

[38] Householder, A., A. Manion, L. Pesante, G. M. Weaver, and R. Thomas (2001). Managing the Threat of Denial-of-Service Attacks. Available at http://www . cert.org/archive/pdf/Managing_DoS.pdf. CERT.

[39] Hu, Y., H. Choi, and H. Choi (2004). Packet Filtering to Defend FloodingBased DDoS Attacks. In 2004 IEEE Sarnoff Symposium, pp. 39-42. Princeton, NJ.

[40] Hussain, A., J. Heidemann, and C. Papadopoulos (2003). A Framework for Classifying Denial of Service Attacks. In SIGCOMM '03, pp. 99-110. Karlsruhe, Germany.

[41] Jin, C., H. Wang, and K. G. Shin (2003). Hop-Count Filtering: An Effective Defense Against Spoofed DoS Traffic. In 10th ACM International Conference on Computer and Communications Security (CCS), pp. 30-41. Washington, DC.

[42] Kai, C., H. Xiaoxin, and H. Ruibing (2004). DDoS Scouter: A Simple IP Traceback Scheme. In Cryptography: 25 years of Cryptography in China, volume 769 of The International Series in Engineering and Computer Science, pp. 217-228.

[43] Kuznetsov, V., A. Simkin, and H. Sandström (2002). An Evaluation of Different IP Traceback Approaches. Lecture Notes in Computer Science, 2513.

[44] Lakshminarayanan, K., D. Adkins, A. Perrig, and I. Stoica (2004). Taming IP Packet Flooding Attacks. SIGCOMM Computer Communications Review, 34(1), pp. 45-50.

[45] Lau, F., S. H. Rubin, M. H. Smith, and L. Trajković (2000). Distributed Denial of Service Attacks. In 2000 IEEE International Conference on Systems, Man, and Cybernetics, volume 3, pp. 2275-2280. Nashville, TN. 
[46] Laufer, R. P., P. B. Velloso, D. de O. Cunha, I. M. Moraes, M. D. D. Bicudo, and O. C. M. B. Duarte (2005). A New IP Traceback System Against Denialof-Service Attacks. In 12th International Conference on Telecommunications (ICT'05). Capetown, South Africa.

[47] Leiwo, J., T. Aura, and P. Nikander (2000). Towards Network Denial of Service Resistant Protocols. In Fifteenth Annual Working Conference on Information Security for Global Information Infrastructures, pp. 301-310. Deventer, The Netherlands.

[48] Lemon, J. (2002). Resisting SYN Flood DoS Attacks with a SYN Cache. In USENIX BSD Conference, pp. 89-97.

[49] Li, J., M. Sung, J. Xu, and L. Li (2004). Large-Scale IP Traceback in HighSpeed Internet: Practical Techniques and Theoretical Foundation. In 2004 IEEE Symposium on Security and Privacy (SEP'04), pp. 115-129.

[50] Lin, H., Z. Zhao, H. Li, and Z. Chen (2002). A Novel Graph Reduction Algorithm to Identify Structural Conflicts. In Proceedings of the 35th Hawaii International Conference on System Sciences (HICSS).

[51] Meadows, C. (1999). A Formal Framework and Evaluation Method for Network Denial of Service. In 12th Computer Security Foundations Workshop (CSFW '99). Mordano, Italy.

[52] Mirkovic, J., G. Prier, and P. L. Reiher (2002). Attacking DDoS at the Source. In 10th IEEE International Conference on Network Protocols (ICNP '02), pp. 312-321. IEEE Computer Society, Washington, DC, USA.

[53] Mofya, E. C. and J. C. Smith (2004). The Optimal Deployment of Filters to Limit Forged Address Attacks in Communication Networks. Lecture Notes on Computer Science, 3073, pp. 239-251. 
[54] Mofya, E. C. and J. C. Smith (2005). Algorithms for the Generalized Minimum Filter Placement Problem on Special Structures. Working Paper. The University of Arizona, Tucson, AZ.

[55] Moore, D., G. M. Voelker, and S. Savage (2001). Inferring Internet Denial-ofService Activity. In 10th USENIX Security Symposium. Washington D.C.

[56] Mutaf, P. (1999). Defending Against a Denial-of-Service Attack on TCP. In 2nd International Workshop on Recent Advances in Intrusion Detection (RAID '99).

[57] Nemhauser, G. L. and L. A. Wolsey (1999). Integer and Combinatorial Optimization. John Wiley \& Sons Inc., New York, NY.

[58] Neumann, P. G. (2000). Inside Risks: Denial-of-Service Attacks. Communications of the $A C M, \mathbf{4 3}(4)$, p. 136.

[59] Papadopoulos, C., R. Lindell, J. Mehringer, A. Hussain, and R. Govindan (2003). COSSACK: Coordinated Suppression of Simultaneous Attacks. In DARPA Information Survivability Conference and Exposition, volume 1, pp. 2-13. Washington, DC.

[60] Park, K., H. Kim, B. Bethala, and A. Selcuk (2003). Scalable protection against DDoS and Worm Attacks. Technical report, Purdue University, West Lafayette, IN.

[61] Park, K. and H. Lee (2000). A Proactive Approach to Distributed DoS Attack Prevention Using Route-based Filtering. Technical report, Purdue University, West Lafayette, IN. CSD-TR 00-017.

[62] Park, K. and H. Lee (2001). On the Effectiveness of Probabilistic Packet Marking for IP traceback Under Denial of Service Attack. In IEEE INFOCOM, pp. $338-347$. 
[63] Park, K. and H. Lee (2001). On the Effectiveness of Route-Based Packet Filtering for Distributed DoS Attack Prevention in Power-Law Internets. In $A C M$ SIGCOMM, pp. 15-26.

[64] Paxson, V. (2001). An Analysis of Using Reflectors for Distributed Denialof-Service Attacks. SIGCOMM Computer Communication Review, 31(3), pp. $38-47$.

[65] Peng, T., C. Leckie, and K. Ramamohanarao (2002). Defending Against Distributed Denial of Service Attacks Using Selective Pushback. In Ninth IEEE International Conference on Telecommunications (ICT 2002).

[66] Peng, T., C. Leckie, and K. Ramamohanarao (2003). Protection from Distributed Denial of Service Attack Using History-Based IP Filtering. In IEEE International Conference on Communications (ICC 2003), volume 1, pp. 482486. Anchorage, Alaska.

[67] Sadiq, W. and M. E. Orlowska (2000). Analyzing Process Models Using Graph Reduction Techniques. Information Systems, 25(2), pp. 117-134. ISSN 03064379 .

[68] Sandström, H. (2001). A Survey of the Denial of Service Problem. Technical report, Department of Computer Science and Electrical Engineering, Luleå University of Technology, Luleå, Sweden.

[69] SANS Institute (2000). Egress Filtering v 0.2. Available at http://www. sans . org/y2k/egress.htm.

[70] Savage, S., D. Wetherall, A. Karlin, and T. Anderson (2000). Practical Network Support for IP Traceback. In ACM SIGCOMM, pp. 295-306.

[71] Shallita, A., M. E. Hassan, S. Maalouf, and A. Zouheiry (2004). A Survey of DDoS Defense Mechanisms. In 3rd FEA Student Conference. 
[72] Snoeren, A. C., C. Partridge, L. A. Sanchez, C. E. Jones, F. Tchakountio, S. T. Kent, and W. T. Strayer (2001). Hash-Based IP Traceback. In SIGCOMM '01: Proceedings of the 2001 conference on Applications, Technologies, Architectures, and Protocols for Computer Communications, pp. 3-14. San Diego, CA.

[73] Song, D. X. and A. Perrig (2001). Advanced and Authenticated Marking Schemes for IP Traceback. In Twentieth Annual Joint Conference of the IEEE Computer and Communications Societies INFOCOM '01, volume 2.

[74] Specht, S. M. and R. B. Lee (2004). Distributed Denial of Service: Taxonomies of Attacks, Tools and Countermeasures. In 17th International Conference on Parallel and Distributed Computing Systems, pp. 543-550. San Francisco, CA.

[75] Stephen, J. (2001). The Changing Face of Distributed Denial of Service Mitigation. Technical report, SANS Institute. Available at https://www. cisecurity.org/rr/whitepapers/threats/462.php.

[76] Stohr, E. A. and J. L. Zhao (2001). Workflow Automation: Overview and Research Issues. Information Systems Frontiers, 3(3), pp. 281-296.

[77] Sung, M. and J. Xu (2002). IP Traceback-based Intelligent Packet Filtering: A Novel Technique for Defending Against Internet DDoS Attacks. In 10th IEEE International Conference on Network Protocols ICNP '02, pp. 302-311.

[78] van der Aalst, W. M. P., A. Hirnschall, and H. M. W. E. Verbeek (2002). An Alternative Way to Analyze Workflow Graphs. In CAiSE '02: Proceedings of the 14th International Conference on Advanced Information Systems Engineering, pp. 535-552. Springer-Verlag, London, UK.

[79] WfMC (1999). Workflow Management Coalition Interface 1: Process Definition Interchange Process Model. Available at http://www.wfmc.org/standards/ docs/TC-1016-P_v11_IF1_Process_definition_Interchange.pdf. 
[80] WfMC (1999). Workflow Management Coalition: Terminology \& Glossary. Available at http://www.wfmc.org/standards/docs/TC-1011_term_ glossary_v3.pdf.

[81] Xiang, Y., W. Zhou, and M. Chowdhury (2004). A Survey of Active and Passive Defence Mechanisms against DDoS Attacks. Technical report, School of Information Technology, Deakin University, Australia.

[82] Xiaofeng, Q., H. Jihong, and C. Ming (2004). A Mechanism to Defend SYN Flooding Attack Based on Network Measurement System. In 2nd International Conference on Information Technology: Research and Education (ITRE 2004), pp. $208-212$. 